\title{
ACTIVE AND ULTRASENSITIVE CHEMICAL AND BIOSENSING THROUGH OPTOTHERMALLY GENERATED MICROBUBBLE
}

\author{
Dissertation \\ Submitted to \\ The School of Engineering of the \\ UNIVERSITY OF DAYTON
}

In Partial Fulfillment of the Requirements for

The Degree of

Doctor of Philosophy in Electro-Optics

\author{
By \\ Farzia Karim \\ UNIVERSITY OF DAYTON \\ Dayton, Ohio \\ May 2020

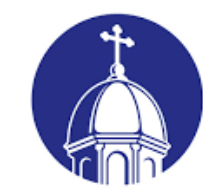 \\ University of \\ Dayton
}


APPROVED BY:

Chenglong Zhao, Ph.D

Advisory Committee Chairman

Assistant Professor

Electro-Optics and Photonics, Physics
Qiwen Zhan, Ph.D

Committee Member

Professor, Electro-Optics and Photonics, Electrical and Computer Engineering
Andy Chong, Ph.D

Committee Member

Associate Professor

Electro-Optics and Photonics, Physics
Erick S. Vasquez, Ph.D

Committee Member

Assistant Professor

Chemical and Materials Engineering
Robert J. Wilkens, Ph.D., P.E.

Associate Dean for Research and Innovation

Professor

School of Engineering
Eddy M. Rojas, Ph.D., M.A., P.E.

Dean, School of Engineering 
(C) Copyright by

Farzia Karim

All rights reserved

2020 


\section{ABSTRACT \\ ACTIVE AND ULTRASENSITIVE CHEMICAL AND BIOSENSING THROUGH OPTOTHERMALLY GENERATED MICROBUBBLE}

Name: Karim, Farzia

University of Dayton

Advisor: Dr. Chenglong Zhao

Rapid and sensitive detection of harmful chemicals or biological samples is essential for medical study and industrial applications. For example, in the food industry, it is an urgent demand to detect hazardous chemicals or pathogens and then efficiently eliminate contaminated sources from the food production chain to protect people from toxic food-borne infection. In addition, toxic chemicals, pesticides and pathogens are also responsible for water, air and soil contamination. Therefore, rapid and sensitive detection of toxic chemicals or pathogens are very important to ensure food safety and evaluating environmental pollution. Most of the sensing systems for detecting chemical or biological samples apply a passive sensing method, in which binding of analytes to the sensor happens through free diffusion. Due to this free diffusion of analytes in a passive sensing method, diffusing process takes several hours even days, especially when the analytes are at low concentrations and therefore suffer from so called diffusion limit. A higher sensitivity can be achieved in passive sensing methods, with nanoscale sensors but at a cost of reduced speed and vice versa. Therefore, speed and sensitivity of sensing need to be traded off. Active sensing methods, in which analytes are actively concentrated towards the sensor, can be used to break the diffusion limit of sensing and significantly improve the sensitivity. In this work, an ultrasensitive and cost-effective chemical and biosensing platform has been developed under ambient condition to demonstrate active sensing of analytes. This method 
works based on an optothermally generated microbubble (OGMB). OGMB is a micronsized bubble that is generated on a liquid-solid interface through laser heating. Due to the strong convective flow induced by OGMB, nanoparticles or analytes can be attracted towards the OGMB and deposited on the surface of a substrate. We have successfully fabricated nanogap-rich structures by generating an OGMB on a glass substrate. The nanoparticles in the nanogap-rich structures form many nanogaps that are ideal for surface enhance Raman scattering (SERS) due to the plasmonic resonance of nanogap-rich structures. Analytes for example any chemical or biological samples can be actively concentrated on the nanogap-rich structure for SERS detection. The OGMB-assisted active sensing method can improve the detection limit of analytes by an order of magnitude compared to that with a passive sensing and thus overcome the diffusion limit of conventional passive sensing methods. Therefore, we expect that, OGMB-assisted active sensing method will find potential applications in advanced chemical and bio-sensing. 
Dedicated to my mother who is my pride, inspiration, strength and my life. 


\section{ACKNOWLEDGMENTS}

At the beginning, all the praise to Almighty for giving me the capability for successful completion of this work. I would like to express my sincere indebtedness to all who have given me the chance to finish this research. Without their generous support this dissertation would not have been completed.

At first, I would like to offer my deepest gratitude to my advisor Dr. Chenglong Zhao who always supported, guided me throughout this work. Moreover, his valuable advices, guidance and comments always encourage me to do hard work and develop a very good understanding, responsibility, knowledge to finish this dissertation. I would like to thank Dr. Zhao for giving me the financial support through National Science Foundation (NSF) and STEM Catalyst Grant of University of Dayton to perform this research.

I would like to express my sincere gratitude to my committee member, Dr. Qiwen Zhan for his valuable advice, guidance and suggestions during my $\mathrm{PhD}$ study that always motivated me to be hard-working, dedicated and passionate about my research.

I am thankful to my committee member, Dr. Andy Chong for his informative and valuable suggestions during my $\mathrm{PhD}$ study.

I would like to offer my gratitude and appreciations to Dr. Erick Vasquez from Chemical and Materials Engineering department and Dr. Yvonne Sun from Biology department of University of Dayton for their enthusiastic initiative, keen interest in my research. I would like to express my thanks to Dr. Vasquez and Dr. Sun for their supervision, valuable suggestions, lab facilities of their department and encouragement throughout this work. I would like to specially thank Dr. Vasquez, Dr. Sun and their 
student, Robert to run a successful collaborative project through 'STEM Catalyst Grant' which we have successfully finished with several publications.

I would like to thank, Vikram and Barbara from NEST lab, University of Dayton for giving me an opportunity to use Scanning Electron Microscope (SEM), Transmission Electron Microscope (TEM) and Raman Spectrometer of NEST lab for my research.

I would like to acknowledge graduate academic affairs of University of Dayton for giving me the financial support during the Summer in 2017, 2018 and 2019 through graduate student summer fellowship 2017, 2018 and 2019.

I am glad to give special thanks to all the group members of Nanophotonic and Nanomanufacturing Lab, University of Dayton for their support and cooperation to make this a blissful journey.

Last but not least, I am grateful to my whole-hearted family, my parents, spouse, my daughter, sisters for their love, well wishes, support, and sacrifices throughout my academic career. I would like to especially thank to my spouse, Shah for his endless support and inspiration during my entire PhD study at University of Dayton. 


\section{TABLE OF CONTENTS}

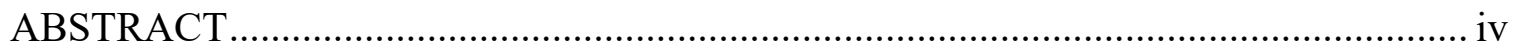

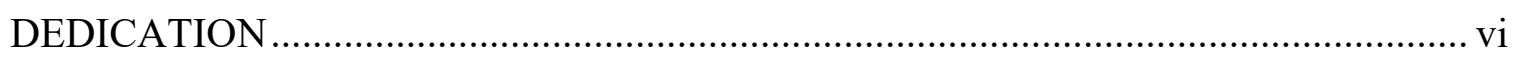

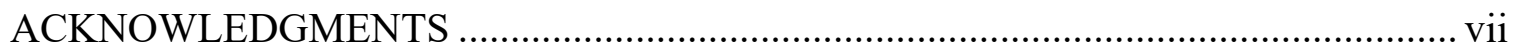

LIST OF FIGURES …................................................................................

LIST OF ABBREVIATIONS AND NOTATIONS …..........................................

CHAPTER 1 INTRODUCTION ....................................................................... 1

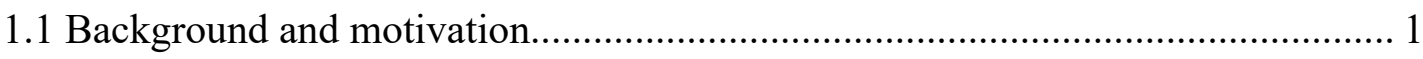

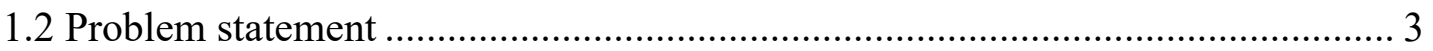

1.3 Mechanism of OGMB-based active sensing …........................................... 5

1.4 Significance and unique features of this work.............................................. 6

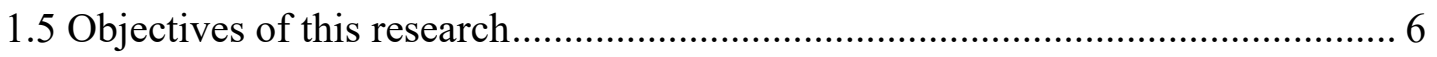

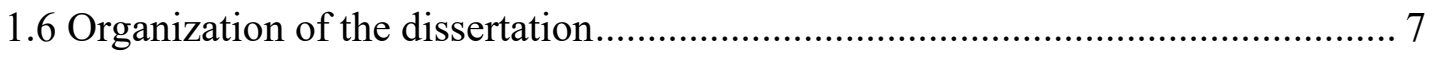

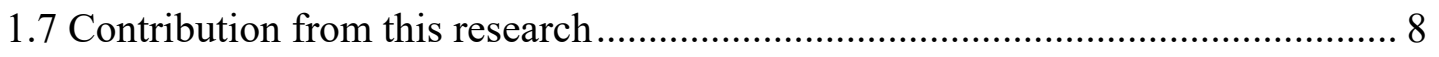

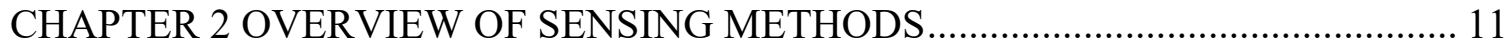

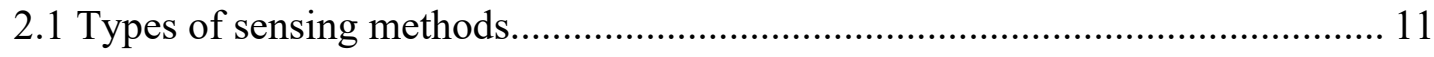

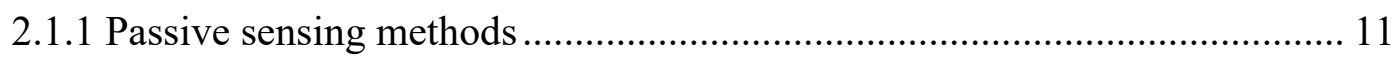

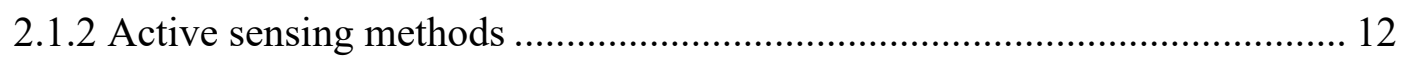

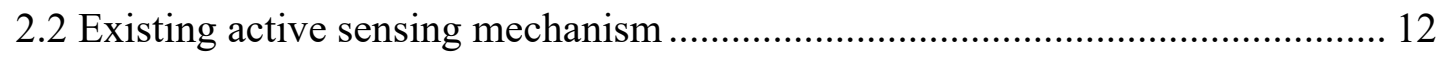

2.3 Sensing methods based on Raman spectroscopy …................................... 16

2.3.1 Basic principle of Raman spectroscopy .............................................. 16 
2.3.2 Basic principle of SERS.

2.3.3 SERS substrates and SERS measurement...................................................... 21

2.4 Active sensing used in this research and questions to be addressed ................... 24

CHAPTER 3 BASIC PRINCIPLE AND APPLICATION OF OGMB ……………….... 25

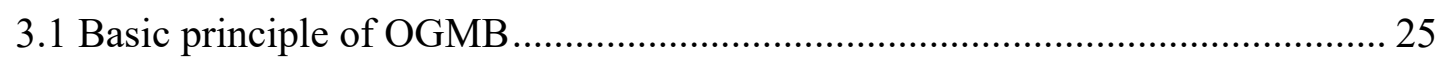

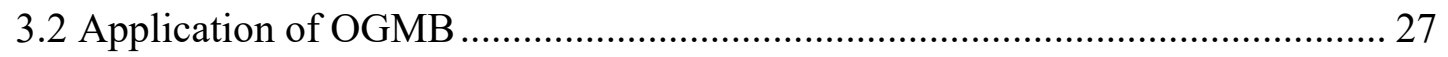

CHAPTER 4 FABRICATION OF NANOGAP-RICH STRUCTURES ......................... 33

4.1 Substrate preparation ....................................................................................... 33

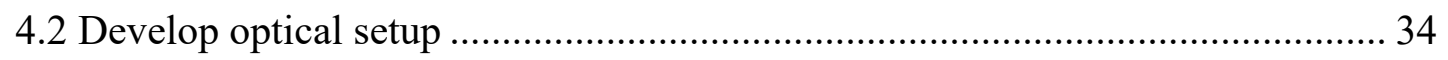

4.3 Microbubble generation through laser heating .................................................. 35

4.4 Fabrication of nanogap-rich structure through OGMB ………………................ 36

4.4.1 Fabrication of gold-nanosphere-structure ................................................... 37

4.4.2 Fabrication of gold-nanoshell-structure …………..................................... 41

4.4.3 Fabrication of gold-nanostars-structure ………………................................ 42

4.5 Fabrication parameters affecting the size of nanogap-rich structures ................. 43

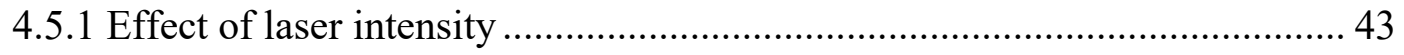

4.5.2 Effect of laser exposure time ……............................................................. 44

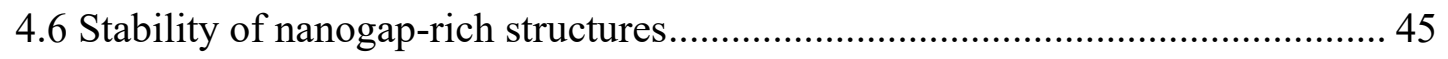

4.7 Effect of gold-coating on substrate for nanogap-rich structures fabrication ....... 45

CHAPTER 5 OGMB BASED NANOGAP-RICH STRUCTURES FOR CHEMICAL

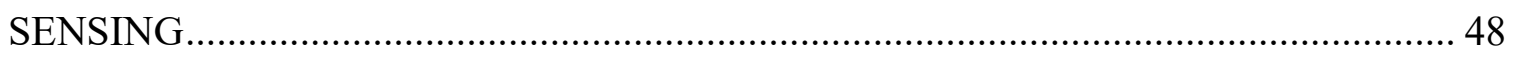

5.1 Develop an ultrasensitive chemical and biosensing platform .............................. 48 
5.2 Nanogap-rich structures for active and ultrasensitive chemical sensing....

5.2.1 Gold-nanosphere-structure for passive chemical sensing

5.2.2 Gold-nanosphere-structure for active chemical sensing 52

5.2.3 SERS enhancement factor of gold-nanosphere-structure due to passive sensing. 56

5.2.4 Enhancement factor of gold-nanosphere-structure due to active sensing..... 57

5.2.5 Gold-nanoshell-structure for passive chemical sensing. 59

5.2.6 Gold-nanoshell-structure for active chemical sensing 60

5.2.7 SERS enhancement factor of gold-nanoshell-structure due to passive sensing. 62

5.2.8 Enhancement factor of gold-nanoshell-structure due to active sensing........ 63

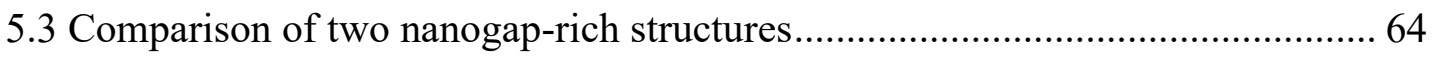

5.3.1 Electric field profile of two nanogap-rich structures ............................... 64

5.3.2 SERS spectra of R6G collected by two nanogap-rich structures..................66

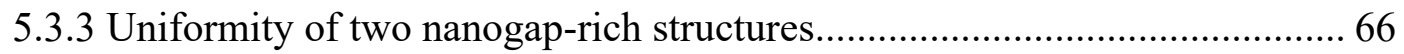

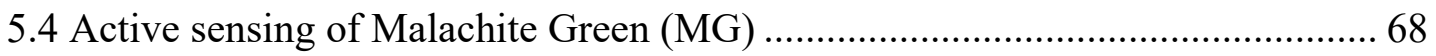

CHAPTER 6 NANOGAP-RICH STRUCTURE FOR BIOSENSING

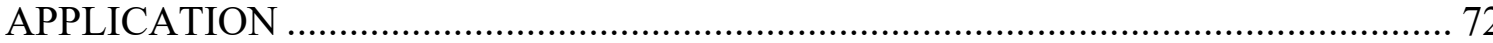

6.1 Listeria monocytogenes detection by nanogap-rich structures ....................... 72

6.1.1 Performance of gold-nanoshell-structure and gold-nanostar-structure......... 73

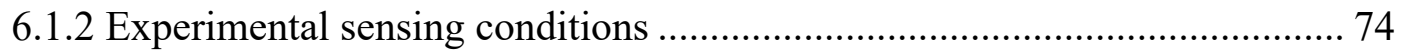


6.2 SERS enhancement factor of gold-nanostar-structure.

6.3 Limitation of active sensing method for biosensing application....................... 78

CHAPTER 7 REAL TIME ACTIVE SENSING OF ANALYTES BY OGMB-

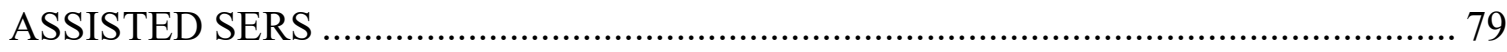

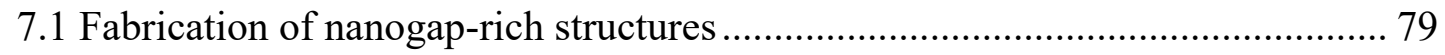

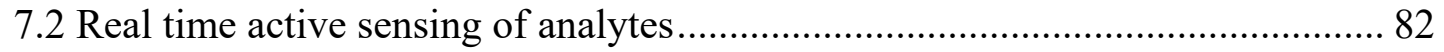

CHAPTER 8 CONCLUSION AND FUTURE WORK …........................................ 85

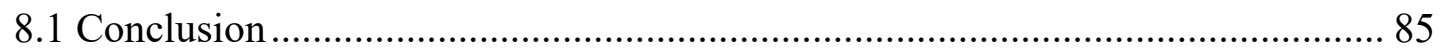

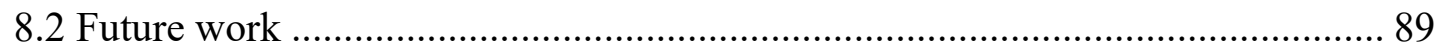

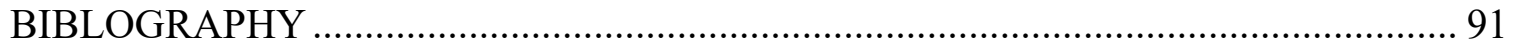




\section{LIST OF FIGURES}

Figure 1.1 (a) Food safety is affected by pathogens, toxic chemicals ${ }^{2}$ and effect of food-borne illness in the $\mathrm{US}^{3}$ (b) air, water and soil are polluted by toxic chemicals, pathogens, heavy metals and the corresponding health effects due to the environmental pollution. ${ }^{4}$

Figure 1.2 SERS, an effective label-free tool used for the detection of (a) different pollutants from a polluted sample ${ }^{18}$ (b) pesticide residues attached to an apple. ${ }^{19}$ 4

Figure 1.3 Flowchart of OGMB-based fabrication of nanogap-rich structure and active analytes sensing. ${ }^{22,23}$. 5

Figure 2.1 Schematic of (a) passive sensing method (b) active sensing method. 12

Figure 2.2 Analytes are localized by a SERS active structures through the combination of superhydrophobic artificial surfaces and plasmonic nanostructures (b) SEM image of hydrophobic surface which is made of periodical silicon micropillar arrays (c) almost constant contact angle measurement of droplet during evaporation at four different times. ${ }^{31}$

Figure 2.3 Schematic of a slippery liquid-infused porous surfaces (SLIPS) offers pinning-free substrate for enriching analytes and helping consequent SERS detections. ${ }^{37}$

Figure 2.4 Schematic of (a) flow-through nanohole array-based sensing and fluorescence image of gold film with nanohole array ${ }^{39}$ (b) plasmonic nanostructure serve as a sensor to actively control the concentration of analytes under an electric field. ${ }^{38}$

Figure 2.5 Different types of nano-sensors consist of free-moving nanoparticles along with surface modifications (a) nanohole arrays coated with $20 \mathrm{~nm}$ silica; (b) nanodisks embedded in optical epoxy coated with $10 \mathrm{~nm}$ silica; (c) Ag nanocubes coated with thin silica film; (d) SEM images of 6 pixels of nanohole arrays for multiplexed biosensing and lens-free imaging. ${ }^{44}$..

Figure 2.6 Electrothermoplasmonic (ETP) effect applied on a localized surface plasmon resonance (LSPR) sensing chip induces an opto-electrical fluid convective flow to concentrate analytes on the sensor surface for active analytes detection. ${ }^{45}$

Figure 2.7 (a) Energy-level diagram showing the states involved in Raman spectra. (b) comparison of stokes and anti-strokes scattering with respect of incident wavelength. ${ }^{51}$

Figure 2.8 (a) Schematic of a Raman spectrometer (b) Raman spectrum of phenylalanine crystals. ${ }^{54}$.... 
Figure 2.9 (a) Weak Raman scattering without metal nanostructure roughness (without SERS) (b) strong Raman scattering with metal nanostructure roughness (with SERS). ${ }^{17}$

Figure 2.10 (a) Schematic of a SERS measurement; (b) comparison of the detection of an analyte, Rhodamine 6G (R6G) with and without SERS. ${ }^{17}$

Figure 2.11 SEM image of different types of SERS substrates manufactured by several methods. (a) Au coated polymer nanofingers SERS manufactured by focused ion beam lithography ${ }^{63}$ (b) Au triangular nanoprism SERS manufactured by e-beam lithography ${ }^{64}$ (c) AuNSs SERS by nanolithography ${ }^{65}$ (d) self-assembled gold nanosphere arrays ${ }^{66}$ (e) aluminum nanocrystals ${ }^{67}$ fabricated by chemical synthesis (f) AuNPs SERS by pen-and-paper approach. ${ }^{68}$

Figure 3.1 (a) Schematic of natural and Marangoni convective flow associated with microbubble generation through the continuous wave $(\mathrm{CW})$ laser heating and rapid deposition of nanoparticles on the surface of substrate due to convective flow ${ }^{80}$ (b) quantum dots (Q-dots) are attracted towards microbubble because of strong convective flow induced by $\mathrm{CW}$ laser heating ${ }^{81}$ (c) a pattern structure formed through microbubble printing of Q-dots. ${ }^{81}$

Figure 3.2 (a) 2D temperature distribution and (b) corresponding velocity field around a microbubble at fixed $373 \mathrm{~K}$ temperature (c) convective velocities at the center and side of microbubble as a function of microbubble temperature (T bubble) ${ }^{79}$

Figure 3.3 (a-c) Temporal distribution of microbubble-generation (d) schematic of microbubble generation and convective flow pattern (e-g) three types of pattern structures; (e) ring (f) square lattice (g) swiss roll developed through optothermal microbubble generation. ${ }^{84}$

Figure 3.4 (a) Schematic illustration of Bubble-Pen-Lithography (BPL) ${ }^{85}$ (b) simulated profile of Marangoni convection around a $1 \mu \mathrm{m}$ microbubble ${ }^{85}$ (c,d) SEM image of (c) patterned PS beads at different laser intensity; scale bar $5 \mu \mathrm{m}$ (d) 3-D structure of aggregated PS beads; scale bar $1 \mu \mathrm{m}$ (e) "BPL" and (f) "UT" pattern from PS beads $(\mathrm{g}, \mathrm{h})$ dark-field optical image of $(\mathrm{g}) 4 \times 4$ array of 3D hollow structures and (h) "SP" pattern of PS beads" ${ }^{85}$; scale bar in (e-h) $10 \mu \mathrm{m}$ (i-k) "Mona Lisa" pattern of red QDs with fluorescence lifetime mapping ${ }^{86}$ (1) fluorescence image of U.S. map depicting states of Texas, Pennsylvania, California printed with different $\mathrm{QDs}^{86}(\mathrm{~m})$ bright-field and dark-field optical image of an Ag ring array fabricated through BPL; scale bar $10 \mu \mathrm{m}$ (n) SERS spectra of different concentration of $\mathrm{R} 6 \mathrm{G}$ collected by Au ring array on AuNIs substrate. ${ }^{71}$

Figure 3.5 (a) Temporal distribution of microbubble generation from Q-dots (b) microscope image of ring array fabricated from Q-dots; scale bar $5 \mu \mathrm{m}$ (c) SEM image of fabricated ring array of Q-dots on a gold film ${ }^{87}(\mathrm{~d})$ pattern structure of Q- 
dots (e) magnified image of part (d) marked by a box (f) schematic of a ring formation through microbubble generation on gold film. ${ }^{87}$.

Figure 3.6 (a) Controlled trapping and accumulation of EVs towards the sensor surface through OGMB. (b) Raman spectra of EVs collected at different condition; EVs clustered (red line), EVs not clustered (black line), EVs with AuNPs clustered $(\text { red line })^{88}$.

Figure 4.1 Schematic of (a) glass coverslip coated with $10 \mathrm{~nm}$ gold film (b) glass cover slip without any gold coating. 34

Figure 4.2 (a) Schematic of experimental setup for optothermal microbubble generation. ${ }^{22}$ L: lens; BS: beam splitter; DM: dichroic mirror; O: objective lens; TL: tube lens; CCD: charge coupled device; ND: variable neutral density filter; PM: power meter. Inset: optical image of an OGMB generated inside the droplet of nanoparticles solution (b) real experimental setup.

Figure 4.3 (a) Optical image of a nanoliter droplet heated by laser beam (b) an OGMB generated inside a nanoliter droplet of nanoparticles solution. ${ }^{22}$ (c) schematic of optothermal microbubble generation and nanoparticles deposition on the substrate due to convective flow induced by OGMB 36

Figure 4.4 Optical image of a nanoliter droplet of gold nanosphere solution (a) before and (b) after generation of an optothermal microbubble inside the droplet of gold nanosphere. ${ }^{22}$ (c) a nanoliter injector known as 'Nanoliter 2010' which was used to create nanoliter droplet of gold nanosphere.

Figure 4.5 (a) Schematic of convective flow pattern induced by an OGMB formed inside a droplet. (b) optical image of a nanogap-rich structures fabricated from a nanoliter droplet of $200 \mathrm{~nm}$ gold nanospheres on a gold-coated glass substrate (c) SEM image of the corresponding area which is marked in (b) by a white box. (d) schematic of radial flow pattern induced by natural evaporation of a sessile droplet of gold nanosphere. (e) optical image of the pattern developed on the same substrate due to the coffee-ring effect induced by the natural evaporation of a sessile droplet of gold nanosphere. (f) SEM image of the corresponding area which is marked in (e) by a white box. ${ }^{22}$

Figure 4.6 (a) TEM image of $200 \mathrm{~nm}$ gold-nanospheres (b) optical image of a nanogap rich structure fabricated from a microliter droplet of $200 \mathrm{~nm}$ gold nanospheres (c) SEM image of the corresponding area of nanogap-rich structure marked in (b) by a white box. ${ }^{23}$

Figure 4.7 (a) TEM image of $240 \mathrm{~nm}$ gold-nanoshells consist of $200 \mathrm{~nm}$ silica core coated by $20 \mathrm{~nm}$ gold nanospheres (b) optical image of a nanogap-rich structure fabricated by $240 \mathrm{~nm}$ gold- nanoshells (c) SEM image of the area of nanogap-rich structure marked in (b) by a white box. ${ }^{23}$ 
Figure 4.8 (a) Optical image of a nanogap rich structure fabricated from $100 \mathrm{~nm}$ gold nanostars (b) SEM image of the corresponding area of nanogap-rich structure marked in (a) by a white box.

Figure 4.9 Size of nanogap-rich structure as a function of laser intensity. The experimental data with error bars are linearly fitted by a solid line. ${ }^{22}$.

Figure 4.10 Size of nanogap-rich structure as a function of laser exposure time. The experimental data with error bars are linearly fitted by a solid line.

Figure 4.11 Nanogap-rich structure (a) before sonication (b) after 10 minutes sonication. ${ }^{23}$

Figure 4.12 Nanogap-rich structure fabricated from $200 \mathrm{~nm}$ gold-nanosphere on a cover glass (a) coated with $10 \mathrm{~nm}$ gold film (b) without gold coating. ${ }^{23}$

Figure 4.13 Simulated profile of temperature distribution of $200 \mathrm{~nm}$ goldnanosphere on a glass coverslip substrate (a) with gold-coating (b) without goldcoating. Simulation was performed by COMSOL Multiphysics. ${ }^{23}$

Figure 5.1 Schematic of ultra-sensitive chemical and biosensing platform. 48

Figure 5.2 Schematic of free diffusion of R6G molecules towards nanogap-rich structure: an example of passive sensing. ${ }^{23}$

Figure 5.3 Renishaw inVia Reflex Micro-Raman used for the SERS measurement of R6G molecules. Inset: SERS spectra of R6G recoded by this Raman spectrometer.

Figure 5.4 (a) SERS spectra of R6G at different concentrations (from $100 \mathrm{nM}$ to 1 $\mathrm{pM}$ ) collected by gold-nanosphere-structure through passive sensing method. Inset: schematic of a gold nanoshere used for the fabrication of gold-nanosphere-structure (b) magnified image of SERS spectra of R6G at lower concentrations (from 100 pM to $1 \mathrm{pM}$ ) marked in (a) by an arrow. Inset: schematic of passive sensing methods. ${ }^{23}$

Figure 5.5 (a) Schematic of R6G accumulation on a gold film through an OGMB. (b) optical image of R6G concentrated on gold film through the generation of an OGMB from R6G solution. ${ }^{23}$

Figure 5.6 Schematic of actively guide and concentrate R6G molecules towards nanogap-rich structure through an OGMB: an example of active sensing. ${ }^{23}$

Figure 5.7 (a) SERS spectra of R6G at different concentrations (from $10 \mathrm{nM}$ to 100 $\mathrm{fM}$ ) collected by gold-nanosphere-structure under active sensing method. Inset: schematic of a gold nanoshere used for the fabrication of gold-nanosphere-structure (b) magnified image of SERS spectra of R6G at lower concentrations (from $10 \mathrm{pM}$ 
to $100 \mathrm{fM}$ ) marked in (a) by an arrow. Inset: schematic of active sensing methods. ${ }^{23}$

Figure 5.8 Normalized SERS intensity of R6G at $1364 \mathrm{~cm}^{-1}$ as a function of R6G concentration through active sensing (solid line) and passive sensing (dashed line) methods with error bars indicating experimental standard deviations. ${ }^{23}$.

Figure 5.9 Normalized Raman intensity of R6G molecules at $1364 \mathrm{~cm}^{-1}$ without gold-nanosphere-structure (blue curve) and with gold-nanosphere-structure (red curve). Inset (top) illustrates magnified Raman spectrum of $10 \mathrm{mM} \mathrm{R6G}$ without gold-nanosphere-structure. Inset (bottom) demonstrates passive sensing is used to obatain these data. ${ }^{23}$

Figure 5.10 Enhancement factor of gold-nanosphere-structure as a function of R6G concentrations due to active sensing of analyte, $\mathrm{R} 6 \mathrm{G} .{ }^{23}$. 58

Figure 5.11 (a) SERS spectra of different concentrations (from $1 \mathrm{nM}$ to $10 \mathrm{fM}$ ) of R6G collected by gold-nanoshell-structure through passive sensing. Inset: schematic of a gold nanoshell used for the fabrication of gold-nanoshell-structure (b) magnified image of SERS spectra of R6G corresponding to lower concentrations (from $1 \mathrm{pM}$ to $10 \mathrm{fM}$ ) marked in (a) by an arrow. Inset: schematic of passive sensing illustrates free diffusion of analytes towards nanogap-richstructure. ${ }^{23}$

Figure 5.12 (a) SERS spectra of R6G at different concentration (from $1 \mathrm{nM}$ to 1 fM) collected by gold-nanoshell-structure through active sensing method. Inset: schematic of a gold nanoshell used for the fabrication of gold-nanoshell-structure (b) magnified image of SERS spectra of R6G at lower concentrations (from $1 \mathrm{pM}$ to $1 \mathrm{fM}$ ) as indicated in (a) by an arrow. Inset: schematic of active sensing of analytes demonstrates analytes are actively guided and concentrated towards nanogap-rich-structure. ${ }^{23}$

Figure 5.13 Normalized SERS intensity of R6G at $1364 \mathrm{~cm}^{-1}$ as a function of R6G concentration by active sensing (solid line) and passive sensing (dashed line). ${ }^{23}$ 61

Figure 5.14 Normalized Raman intenisty of R6G at $1364 \mathrm{~cm}^{-1}$ without goldnanoshell-structure (blue curve) and with gold-nanoshell-structure (red curve). Inset (top) depicts magnified Raman spectrum of $10 \mathrm{mM}$ R6G without goldnanoshell-structure. Inset (bottom) illustrates schematic of passive sensing method is applied to record these data. ${ }^{23}$

Figure 5.15 Enhancement factor of gold-nanoshell-structure as a function of R6G concentrations due to active sensing. ${ }^{23}$

Figure 5.16 Simulated electric field profile of (a) gold nanosphere array (b) gold nanoshell (silica core) array at an exciting laser wavelength of $785 \mathrm{~nm}$. Inset: schematic structure of (a) gold nanosphere array (b) gold nanoshell (silica core) array. ${ }^{23}$ 
Figure 5.17 SERS spectrum of $1 \mu \mathrm{M} \mathrm{R6G}$ recorded by gold-nanosphere-structure and gold-nanoshell-structure. Inset: schematic of a gold-nanosphere and goldnanoshell. ${ }^{23}$.

Figure 5.18 $(a, b)$ Histogram of SERS intensity variation of 100 pM R6G at 1364 $\mathrm{cm}^{-1}$. SERS spectra of R6G were collected from 15 different spots of a (a) goldnanosphere-structure (b) gold-nanoshell-structure. ${ }^{23}$

Figure 5.19 (a) SERS spectra of different concentrations (from $1 \mathrm{nM}$ to $1 \mathrm{fM}$ ) of MG collected by gold-nanoshell-structure through active sensing method. Inset: schematic of a gold-nanoshell used to fabricate gold-nanoshell-structure. (b) magnified image of SERS spectra of lower concentrations (from $1 \mathrm{pM}$ to $1 \mathrm{fM}$ ) of MG marked in (a) by an arrow. ${ }^{23}$

Figure 5.20 Normalized SERS intensity of MG at $1,619 \mathrm{~cm}^{-1}$ as a function of MG concentration by applying active sensing method. ${ }^{23}$......

Figure 5.21 (a) An OGMB which is generated on gold-nanoshell-structure for active sensing of analytes, R6G. (b) normalized SERS intensity of R6G versus the duration of OGMB generation during the active analytes sensing. ${ }^{23}$

Figure 6.1 SERS spectra of different concentration of Listeria collected by (a) goldnanoshell structure (b) gold-nanostar-structure respectively. Inset: schematic of (a) gold-nanoshell (b) gold-nanostar used for the fabrication of gold-nanoshellstructure and gold-nanostar-structure. ${ }^{12}$

Figure 6.2 Schematic of free diffusion of Listeria towards nanogap-rich structure which demonstrates passive sensing of Listeria.

Figure 6.3 Normalized SERS intensity (with error bars) of Listeria at $1345 \mathrm{~cm}^{-1}$ as a function of Listeria concentration. Inset (bottom): schematic of passive sensing method shows free diffusion of Listeria towards nanogap-rich structure. Inset (top): Scanning Electron Microscope (SEM) image of Listeria monocytogenes (Listeria) which was detected by gold-nanostar-structure.

Figure 6.4 Raman intensity of Listeria at $1345 \mathrm{~cm}^{-1}$ without gold-nanostarstructure (blue curve) and with gold-nanostar-structure (red curve). 76

Figure 7.1 (a) Schematic of experimental setup. L: lens; BS: beam splitter; DM: dichroic mirror; O: objective lens; TL: tube lens; CCD: charge coupled device; ND: variable neutral density filter; PM: power meter. Inset: schematic of OGMB formation and gold nanoshells deposition on optical fiber substrate through strong Marangoni convective flow associated with OGMB. (b) real experimental setup at Nanophotonic and Nano-manufacturing (NPNM) lab led by Dr. Zhao.

Figure 7.2 (a) Optical image of an optical fiber core with several nanogap-richstructures fabricated by OGMB (b) SEM image of the corresponding area which is marked in (a) by a white box. 
Figure 7.3 An OGMB generated from R6G molecules during the SERS detection of $\mathrm{R} 6 \mathrm{G}$

Figure 7.4 SERS spectra of (a) $50 \mathrm{mM}$ Urea (b) $50 \mathrm{mM}$ R6G collected by OGMBassisted gold nanoshell-structure fabricated on optical fiber core after removing the background Raman signal of optical fiber as depicted in the inset of (a).

Figure 8.1 Detection and discrimination of pathogen (bacteria) by SERS measurement and principal components analysis (PCA). ${ }^{21}$ 


\section{LIST OF ABBREVIATIONS AND NOTATIONS}

$\begin{array}{ll}\text { OGMB } & \text { Optothermally generated microbubble } \\ \text { SERS } & \text { Surface enhanced Raman scattering/spectroscopy } \\ \text { AuNPs } & \text { Gold nanoparticles } \\ \text { AgNPs } & \text { Silver nanoparticles } \\ \text { AuNIs } & \text { Gold nano-islands } \\ \text { Ag ring AuNIs } & \text { Silver ring array on gold nano-islands } \\ \text { AuNShells } & \text { Gold nanoshells } \\ \text { AuNSs } & \text { Gold nanostars } \\ \text { SEM } & \text { Scanning electron microscope } \\ \text { TEM } & \text { Transmission electron microscope } \\ \text { EF } & \text { Enhancement factor } \\ \text { R6G } & \text { Rhodamine 6G } \\ \text { MG } & \text { Malachite Green } \\ \text { CFU } & \text { Colony forming unit } \\ \text { PCA } & \text { Principal components analysis }\end{array}$




\section{CHAPTER 1}

\section{INTRODUCTION}

\subsection{Background and motivation}

Rapid and accurate detection of hazardous chemicals and pathogens is crucial for medical and industrial applications. For example, in the food industry it is important to detect hazardous chemicals or pathogens which are responsible for toxic food-borne infections. For more than several decades, food safety has become a significant global health concern since food-borne diseases correspond to a widespread and increasing public health problem all over the world. Over the last 20 years, food-borne diseases have been increased significantly in the developed as well as developing countries. ${ }^{1}$
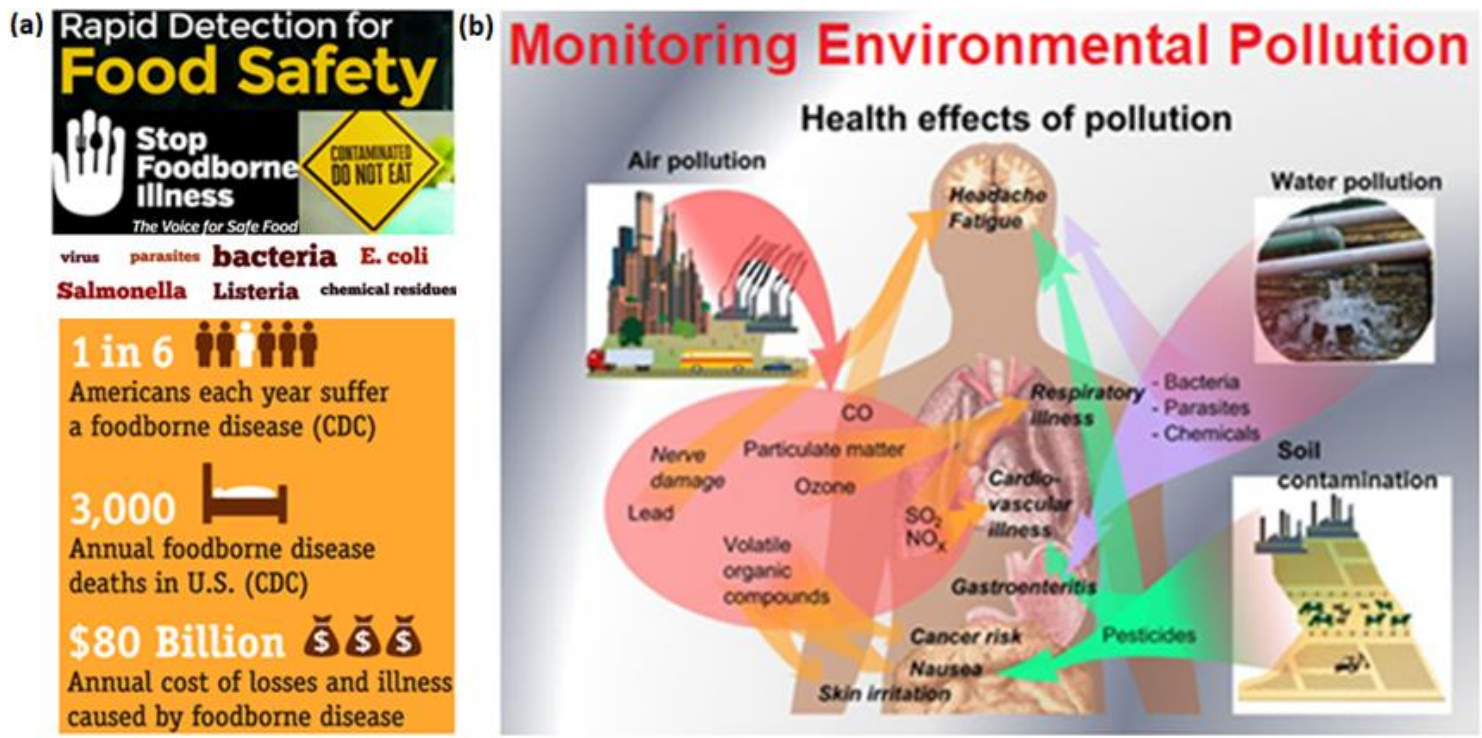

Figure 1.1 (a) Food safety is affected by pathogens, toxic chemicals ${ }^{2}$ and effect of foodborne illness in the $\mathrm{US}^{3}$ (b) air, water and soil are polluted by toxic chemicals, pathogens, heavy metals and the corresponding health effects due to the environmental pollution. ${ }^{4}$

According to the annual health report from the Centers for Diseases Control and Prevention (CDC), around 48 million people in the United States become ill, among them 128000 people are hospitalized, and 3000 people die every year due to food-borne diseases, which 
results a econmonic loss of 80 billion of U.S. dollars. In recent years, around a quarter of U.S. population is at higher risk due to food-borne diseases. ${ }^{5}$ Figure 1.1 (a) represents sufferings of people due to food-borne illness in the U.S. Therefore, there is an increasing demand to ensure the safety of food supply. In addition, world population is expected to increase in near future. Therefore, we need to increase the production of food and develop ways to provide safe food free from pathogenic or chemical contamination.

In the food industries different types of coloring agents are used in the dye beverages, fruits, or candy to make them visually more appealing to the customers. However, due to the carcinogenic nature of some dyes, using them is now banned in some countries. These types of illegal dyes or some harmful food chemicals or biological agents are still used in some of the developing countries due to low level of rules and regulation or lack of proper methods to distinguish these harmful products from visually similar types of dyes. However, when these type of toxic chemicals, pathogens, illegal dyes or any other contaminated sources come in contact with the foods in the food industry, then food safety is seriously affected ${ }^{2}$ as shown in Figure 1.1 (a). Therefore, rapid detection and efficient elimination of harmful chemicals, pathogens or other contaminated sources from the food production chain can prevent food-borne diseases and ensure food safety.

In addition, toxic chemicals, pesticides, and pathogens such as bacteria, parasites, and heavy metals can contaminate air, water, soil and therefore become the cause of environmental pollution. The health effects due to these type of pollutions are very serious. For example, these types of pollutions may cause respiratory and cardio vascular illness, nerve damage and even can be the cause for cancers. ${ }^{4}$ Figure 1.1 (b) demonstrate the potential harmful effects on human body due to the environmental pollution. Therefore, 
rapid and sensitive detection of toxic chemicals or pathogens are very important to ensure food safety, security and evaluating environmental pollution.

\subsection{Problem statement}

Most of the sensing systems for detecting hazardous chemical, pathogens or other biological samples apply a passive sensing method, in which binding of analytes to the sensor happens through free diffusion. Due to this free diffusion of analytes in a passive sensing method, diffusing process takes several hours even days, especially when the analytes are at low concentrations and therefore suffer from so called diffusion limit. In addition, conventional chemical and biosensing methods are generally time-consuming, laborious, expensive and need fluorescent labeling to detect chemical or pathogens which cannot meet the essential need for rapid detection of toxic chemicals and pathogens.

In recent years several label-free sensing methods have been used based on optical, ${ }^{6}$ electrical, ${ }^{7-9}$ and mechanical principle. ${ }^{10-12}$ For example, surface enhanced Raman spectroscopy (SERS) has been used as a powerful characterization tool for rapid and efficient detection and discrimination of toxic chemicals, pathogens or other pollutants. ${ }^{13-}$ 17 and therefore plays an important role in food safety and monitoring environmental pollution. SERS provides structural fingerprint of specific molecule and becomes an ultrasensitive label-free technique to detect chemical and biological species. ${ }^{14-16}$ Figure 1.2 (a) illustrate the detection of different pollutants through the SERS measurement of a polluted sample. ${ }^{18}$ Figure 1.2 (b) depicts the SERS spectrum of pesticide residues attached to an apple which is recoded by a gold nanoparticles (AuNPs) SERS substrate. The AuNPs 
SERS is fabricated on an adhesive tape and used for the SERS measurement of the pesticide residues. ${ }^{19}$ SERS measurement will be described in detail in chapter 2 of this dissertation.

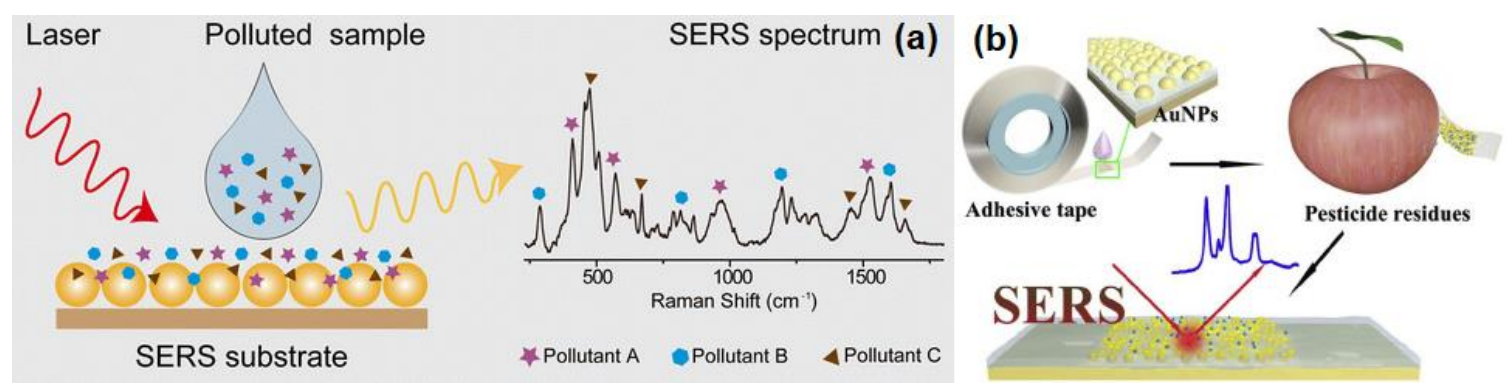

Figure 1.2 SERS, an effective label-free tool used for the detection of (a) different pollutants from a polluted sample ${ }^{18}$ (b) pesticide residues attached to an apple. ${ }^{19}$

SERS is a powerful tool that allows simultaneous multi-component detection and analysis. ${ }^{20,21}$ For example three different types of pollutants are simultaneously detected by SERS as shown in Figure 1.2 (a). Other advantages of SERS includes its simplicity for sample manipulation, speed of analysis, in-situ identification of analytes. ${ }^{20}$ Despite the many advantages of SERS-based sensing, it is still a passive sensing method and thus diffusion-limited.

In order to overcome the diffusion limit of a sensing system, active sensing methods need to be used where the analytes are forced to concentrate towards the sensors. Active sensing method can locally increase the concentration of analytes on the sensor surface and can significantly improve the sensitivity of sensor. In addition, affordable active sensing method not only allows for rapid and sensitive detection of toxic chemical and pathogens but also reduce the cost of conventional chemical and biosensing method. A cost-effective active sensing method has been demonstrated in this work through which chemical and biological samples are rapidly detected by label-free SERS technique. This methods works 
based on an optothermally generated microbubble (OGMB). The mechanism of OGMBassisted active sensing method is described in the following section.

\subsection{Mechanism of OGMB-based active sensing}

OGMB is a micron-sized bubble that is generated on a solid-liquid interface through laser induced heating of a solid surface. The size and position of an OGMB on the solid surface can be remotely controlled by the laser. The OGMB is used not only to rapidly manufacture a SERS substrate under ambient condition but also concentrate analytes towards the SERS substrate for active sensing. Figure 1.3 shows the working principle of OGMB-based active sensing as follows:

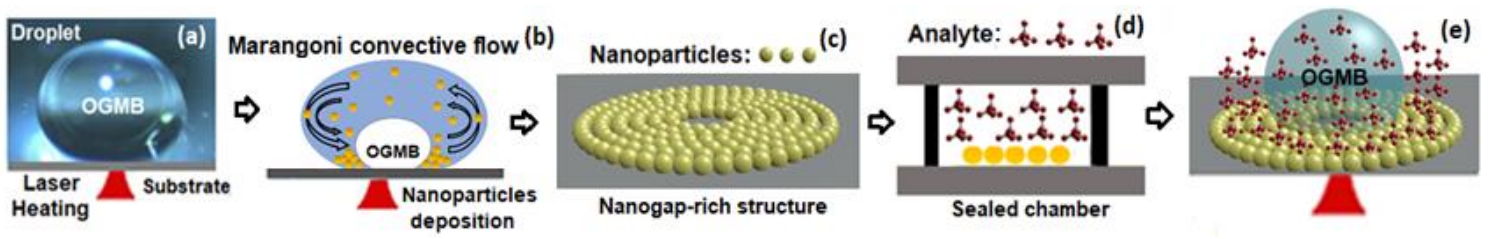

Figure 1.3 Flowchart of OGMB-based fabrication of nanogap-rich structure and active analytes sensing. ${ }^{22,23}$

1) At first a droplet of metallic nanoparticles solution is placed on a cover glass coated with $10 \mathrm{~nm}$ thick gold film. Figure 1.3 (a) shows an optical image of such a droplet on a goldcoated cover glass.

2) A low cost continuous-wave laser is focused on the cover glass and an OGMB is generated inside the droplet of metallic nanoparticles solution. The OGMB creates a strong convective flow known as Marangoni convective flow through which nanoparticles are attracted towards OGMB and rapidly deposited on the surface of gold-coated cover glass as schematically shown in Figure 1.3 (b). 
3) Figure 1.3 (c) schematically shows the SERS substrate consisting of a nanogap-rich structure that is fabricated on a gold-coated cover glass through the rapid deposition of nanoparticles on the cover glass under ambient condition in this method.

4) Analytes such as any chemical or biological sample was taken inside a sealed chamber consists of a cover glass on the top surface and a gold-coated glass with nanogap-rich structure at the bottom surface as shown in Figure 1.3 (d). Analytes with nanogap-rich structure in a sealed chamber serves as an ultrasensitive chemical and biosensing platform.

5) Another OGMB was generated from analytes through laser heating. OGMB associated with strong Marangoni convective flow then guides and concentrates analytes on nanogaprich structure for active analytes sensing as shown in Figure $1.3(\mathrm{e})$.

\subsection{Significance and unique features of this work}

The nanogap-rich structures fabricated from an OGMB are ideal for label-free SERS detection because of the plasmonic resonance of nanogap-rich-structures. ${ }^{24-26}$ An OGMB is not only used to rapidly manufacture a SERS substrate under ambient conditions, but also used to locally increase the concentration of chemical or biological samples near the SERS substrate for active sensing. It provides an affordable way to rapidly and actively detect chemical and biological sample for potential applications in food industry and monitoring environmental pollution.

\subsection{Objectives of this research}

The objective of this work is to develop an affordable OGMB-based active sensing method to overcome the diffusion limit of conventional passive sensing methods. 
The following tasks will be performed:

Task 1: Affordable and rapid fabrication of nanogap-rich-structure through an OGMB to serve as a SERS substrate

Task 2: Concentrate chemicals or biological samples towards the nanogap-rich-structure by an OGMB.

Task 3: Active sensing of analytes based on an OGMB-assisted SERS.

\subsection{Organization of the dissertation}

This dissertation consists of eight chapters which is organized as follows:

Chapter 1 includes background and motivation of this research, statement of problem, significance, unique feature and objectives of this research.

Chapter 2 gives the overview of sensing methods.

Chapter 3 gives the basic principles and application of optothermally generated microbubble (OGMB).

Chapter 4 describes the experimental fabrication method of nanogap-rich structures from metallic nanoparticles solution through an OGMB.

Chapter 5 represents the experimental results of OGMB-assisted nanogap-rich structures for active and ultrasensitive chemical sensing application.

Chapter 6 demonstrates the experimental results of OGMB-based nanogap-rich structures for ultrasensitive biosensing application.

Chapter 7 gives OGMB-based nanogap-rich structure for real time active chemical sensing application.

Chapter 8 provides conclusion and future work of this research. 


\subsection{Contribution from this research}

The following articles, presentation and contributions are endorsed during the completion of this research.

\section{Published articles}

[1] F. Karim, E. Vasquez, Y. Sun, C. Zhao. Optothermal microbubble assisted manufacturing of nanogap-rich structures for active chemical sensing. Nanoscale. $2019 ; 11,20589-20597$

[2] F. Karim, E.S. Vasquez, C. Zhao. Fabricated nanogap-rich plasmonic nanostructures through an optothermal surface bubble in a droplet. Optics Letters. $2018 ; 43(2), 334-336$

[3] F. Karim, T. B. Smith, C. Zhao. Review of optical detection of single molecules beyond the diffraction and diffusion limit using plasmonic nanostructures. Journal of Nanophotonics. 2017; 12(1), 012504

[4] R. T. Busch, F. Karim, J. Weis, Y. Sun, C. Zhao, E. S. Vasquez. Optimization and structural stability of gold nanoparticle-antibody bioconjugates. ACS Omega. 2019, 4 (12), 15269-15279

[5] A. S. Solís, F. Karim, M. S. Alam, Q. Zhan, T. L. Luke, C. Zhao. Print metallic nanoparticles on a fiber probe for 1064-nm surface-enhanced Raman scattering. Optics Letters. 2019, 44, 4997-5000

[6] M.S. Alam, F. Karim, C. Zhao. Single-molecule detection at high concentrations with optical aperture nanoantennas. Nanoscale. 2016; 8, 9480-9487. 


\section{Manuscript under Review Process}

[1] R. T. Busch, F. Karim, Y. Sun, H. Fry, Y. Z. Liu, C. Zhao, and E. S. Vasquez. Pathogen detection and capture with label-free polyclonal antibody gold-coated magnetic nanoshells SERS substrates. ACS Applied Bio Materials, 2020

[2] A. S. Solís, F. Karim, Q. Zhan, T. L. Luke, C. Zhao. Reduced-fluorescence background and repeatable raman sensing with a 1064-nm SERS-on-paper sensor. Optics Express. 2020

\section{Manuscript in Preparation}

[1] F. Karim, M S Alam, C. Zhao. Optothermally generated microbubble assisted nanogap-rich-structure on optical fiber probe for real time active chemical sensing. ACS Sensors, 2020.

[2] F. Karim, C. Zhao. Gold nanoshell SERS on cotton substrate for chemical sensing. Optics Letters, 2020.

\section{Conference and Proceedings}

[1] F. Karim, E. Vasquez, Y. Sun, C. Zhao. Active and ultrasensitive chemical and biosensing through optothermally generated microbubble. Conference on Lasers and Electro-Optics (CLEO), 2020. San Jose, CA, USA. Oral Presentation

[2] F. Karim, C. Zhao. Optothermally generated microbubble for active and ultrasensitive chemical and biosensing. Brother Joseph W. Stander Symposium, 2020. OH, USA. Oral Presentation. 
[3] $\quad$ F. Karim, C. Zhao. Ultrasensitive bio-sensing through bubble in a droplet method. IONS-International OSA Network of Students Midwest, 2018, Purdue University, IN, USA and University of Dayton, OH, USA. Oral Presentation

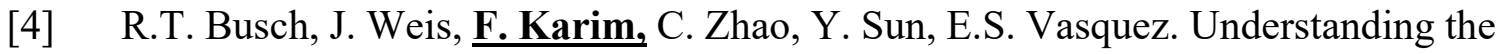
effects of solution conditions on antibodies binding to Au Nanoparticles. Tech Connect World Innovation Conference and Expo, 2019. Boston, MA, USA. Poster Presentation.

[5] J. Soriano, F. Karim, C. Zhao. Identification of a model organism for Giardia Lamblia by Raman spectroscopy. Bulletin of American Physical Society, 2019. Boston, MA, USA. Poster Presentation.

[6] F. Karim, C. Zhao. Bubble-in-a-droplet based surface enhanced Raman scattering substrate for ultra-sensitive chemical and biosensing. Brother Joseph W. Stander Symposium, 2019. OH, USA. Oral presentation.

[7] F. Karim, C. Zhao. Fabricate nanogap-rich plasmonic nanostructures through an optothermal surface bubble in a nanoliter droplet. Brother Joseph W. Stander Symposium, 2018. OH, USA. Oral presentation.

[8] F. Karim, C. Zhao. Cost-effective 3D nano-fabrication technique through optothermal bubbles. Brother Joseph W. Stander Symposium, 2017. OH, USA. Oral presentation. 


\section{CHAPTER 2 \\ OVERVIEW OF SENSING METHODS}

The evaluation of a sensing system depends on two key parameters known as detection sensitivity and time. These are very important but contradictory parameters in a sensing system. For example: signal-to-noise ratio of a sensor can be improved through decreasing the size of active sensing area which will enable ultrasensitive sensing even at an extremely low concentrations of analytes. However, for a smaller active sensing area the analytes will take longer time to diffuse towards the sensor in order to generate an effective sensing signal, especially when the analytes are in a highly diluted solution. ${ }^{27,28}$ For example, it took around one hour for the first analyte to bind on a micro-sensor if the concentration of the analytes is one femtomolar. It will takes even several days if the sensor is at the nanoscales. ${ }^{27}$ In this chapter, we will describe the overview of a sensing method.

\subsection{Types of sensing methods}

Passive or active sensing can be applied based on the way analyte binds on the sensor. The overview of passive and active sensing methods is explained in the following section:

\subsubsection{Passive sensing methods}

Passive sensing is a sensing method in which the binding of analytes takes place through free diffusion, i.e. waiting for the analytes to freely diffuse towards the surface of sensor. The schematic of a passive sensing method is illustrated in Figure 2.1 (a). The passive sensing method works effectively when the analytes are at high concentrations because the analytes have higher chances to interact with the sensor. However, it fails to work 
efficiently at a highly diluted solution because it takes unrealistically long times to diffuse towards the small sensing area of the sensor ${ }^{29-32}$ For example, the detecion limitation of a practical passive sensing is at picomolar-range for a nanoscale sensor. ${ }^{27}$

(a)

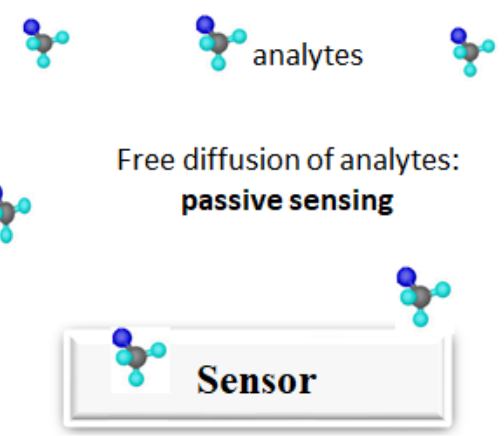

(b) 80 analytes

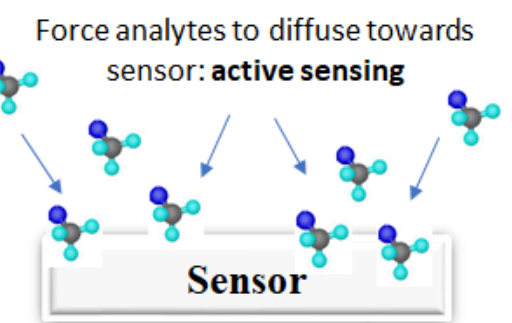

Figure 2.1 Schematic of (a) passive sensing method (b) active sensing method.

\subsubsection{Active sensing methods}

In order to overcome the diffusion limit of a passive sensing system, active sensing can be applied in which the analytes are forced for binding towards the sensor. It can significantly reduce the waiting time for the analytes to diffuse towards the sensor and increase the detection limit by locally increasing the concentration of analytes on the sensor surface as schematically shown in Figure 2.1 (b).

\subsection{Existing active sensing mechanism}

Several active sensing methods have been reported in the literature. For example, a superhydrophobic artificial surface along with an evaporating liquid droplet has been reported by De Angelis et $a l^{31}$ as shown in Figure 2.2 to break the diffusion limit. Droplet along with analytes slide on the superhydrophobic surface as it evaporates without being pinned at the initial contact point as shown in Figure 2.2 (c) ${ }^{33-36}$ The localization and recognition of a single lambda DNA molecule has been demonstrated using this method. 


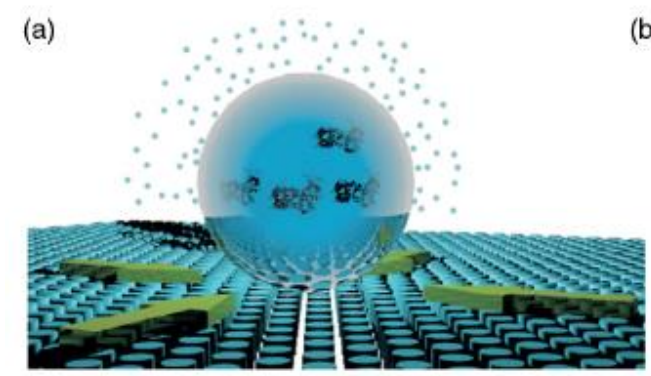

(c)

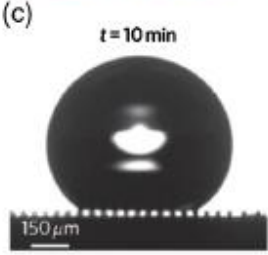

(b)

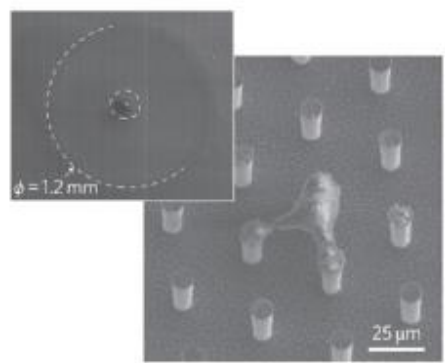

$t=25 \min$ $t=35 \min$

Figure 2.2 Analytes are localized by a SERS active structures through the combination of superhydrophobic artificial surfaces and plasmonic nanostructures (b) SEM image of hydrophobic surface which is made of periodical silicon micropillar arrays (c) almost constant contact angle measurement of droplet during evaporation at four different times. ${ }^{31}$

A slippery substrate which allows free movement of a droplet on a substrate to achieve the similar purposes is demonstrated by Yang et. $a l^{37}$ as shown in Figure 2.3. However, these methods need a superhydrophobic substrate along with nanostructures which are artificially designed through expensive nanofabrication process. Therefore, these methods are limited to a few types of substrates.

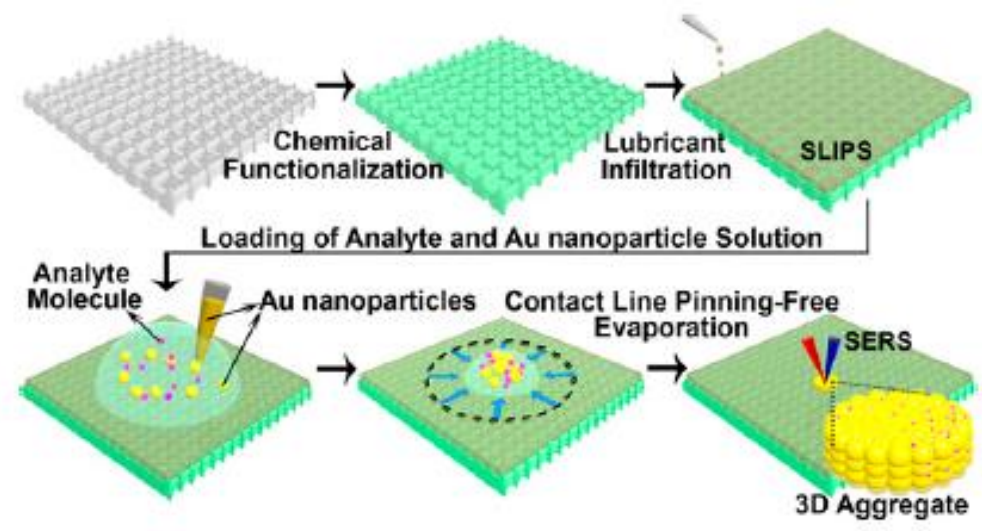

Figure 2.3 Schematic of a slippery liquid-infused porous surfaces (SLIPS) offers pinningfree substrate for enriching analytes and helping consequent SERS detections. ${ }^{37}$ 
Another active sensing method is to actively control the concentration of analytes by forcing the movement of analytes through nanochannels under an external electric field ${ }^{38,39} \mathrm{An}$ array of nanoholes in a gold film combined with a microfluidic system is used which serves not only as a plasmonic sensor but also as a flow-through nanochannel as depicted in Figure 2.4 (a). Electric field is applied across the solution and force analytes to localize towards the sensor as shown in Figure 2.4 (b). However, in this method liquid solution needs to be specially modified to work with an electric field and only applicable for sensors having nanoholes.
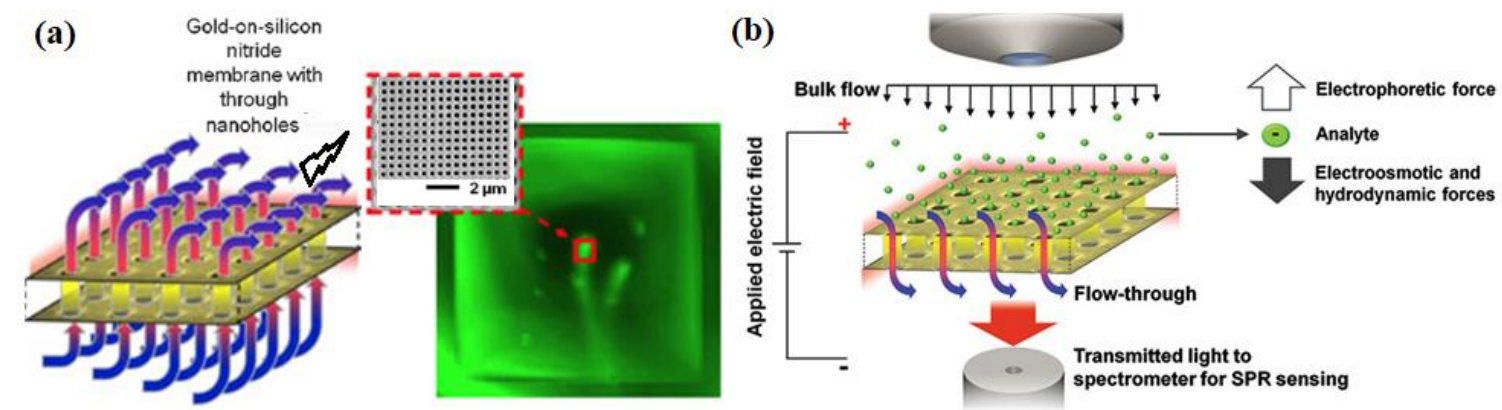

Figure 2.4 Schematic of (a) flow-through nanohole array-based sensing and fluorescence image of gold film with nanohole array ${ }^{39}$ (b) plasmonic nanostructure serve as a sensor to actively control the concentration of analytes under an electric field. ${ }^{38}$

Another active sensing effort is to use nano-sensors consisting of free-moving nanoparticles on a modified surface $e^{40-44}$ to increase the binding rate of analytes on the senor as shown in Figure 2.5. However, this method is still diffusion limited because nano-sensor along with the analytes needs to diffuse towards a small exciting light source for an effective detection of analytes.

Garcia-Guirado et al. demonstrated an active sensing method based on an electrothermoplasmonic (ETP) effect on a localized surface plasmon resonance (LSPR) sensing chip. ${ }^{45}$ 


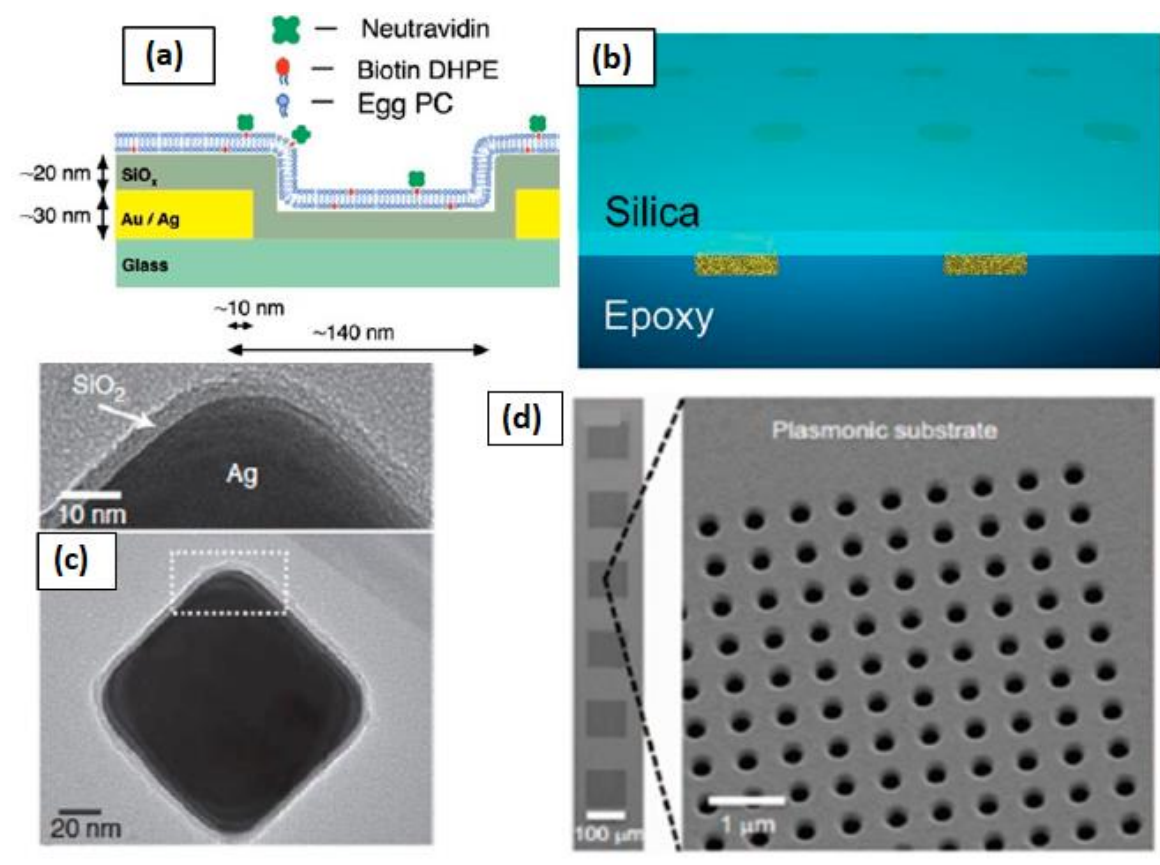

Figure 2.5 Different types of nano-sensors consist of free-moving nanoparticles along with surface modifications (a) nanohole arrays coated with $20 \mathrm{~nm}$ silica; (b) nanodisks embedded in optical epoxy coated with $10 \mathrm{~nm}$ silica; (c) Ag nanocubes coated with thin silica film; (d) SEM images of 6 pixels of nanohole arrays for multiplexed biosensing and lens-free imaging. ${ }^{44}$

This method is capable to detect analytes at lower concentration from a small sample volume to overcome the diffusion limit of biosensing.

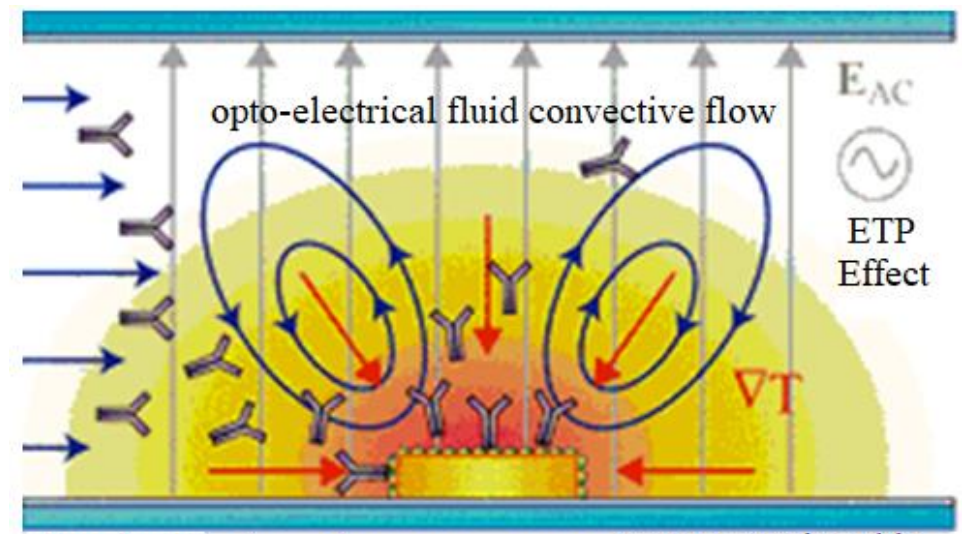

Localized surface plasmon resonance (LSPR) sensing chip

Figure 2.6 Electrothermoplasmonic (ETP) effect applied on a localized surface plasmon resonance (LSPR) sensing chip induces an opto-electrical fluid convective flow to concentrate analytes on the sensor surface for active analytes detection. ${ }^{45}$ 
ETP effect was applied inside a localized surface plasmon resonance (LSPR) sensing chip which induces an opto-electrical fluid convective flow as shown in Figure 2.6 to concentrate analytes on the sensor surface to improve the sensitivity of sensor.However, this method needs multiple steps and high vaccum nanofabrication process to fabricate the sensing chip and thus become time-consuming and expensive method.

Due to the above-mentioned limitations, a cost-effective active sensing method has been demonstrated in this work where analytes are locally concentrated on a surface enhanced Raman scattering (SERS) sensor for active sensing. SERS is a promising tool which is able to significantly improve the natural weak Raman scattering for the efficient detection of an analyte. The sensing method based on Raman spectroscopy and SERS are described in the following section.

\subsection{Sensing methods based on Raman spectroscopy}

\subsubsection{Basic principle of Raman spectroscopy}

Raman spectroscopy, a spectroscopic technique named after Indian physicist C. V. Raman is typically used to determine vibrational modes of molecules. ${ }^{46,47}$ It provides a structural fingerprint of molecules through which molecules can be identified. Raman spectroscopy is a scattering technique in which the frequency of a small fraction of scattered light is different from the frequency of the monochromatic incident light. Usually light from a laser in the visible, near ultraviolet or near infrared range is used in Raman spectroscopy, although X-rays can be also used. It happens due to the inelastic scattering of incident radiation through its interaction with the vibrating molecules. ${ }^{48,49}$ 
In a Raman spectroscopic measurement, a monochromatic laser beam illuminates the sample which interacts with the molecules of the sample and a scattered light is originated. The scattered light with a frequency different from the incident light is known as inelastic scattering. Raman signal arises because of the inelastic collision between the incident monochromatic radiation and molecules of a sample. When the monochromatic incident radiation strikes at the sample, it interacts with the sample molecules and scatters in all directions. Most of the scattered radiation are elastic scattering and has the same frequency of incident radiation. Elastically scattered radiation are referred to as Rayleigh scattering. Only a small fraction (approximately 1 in 10 million photons) of the scattered radiation has the frequency which is different from the frequency of incident radiation and produces Raman scattering. ${ }^{46,50}$
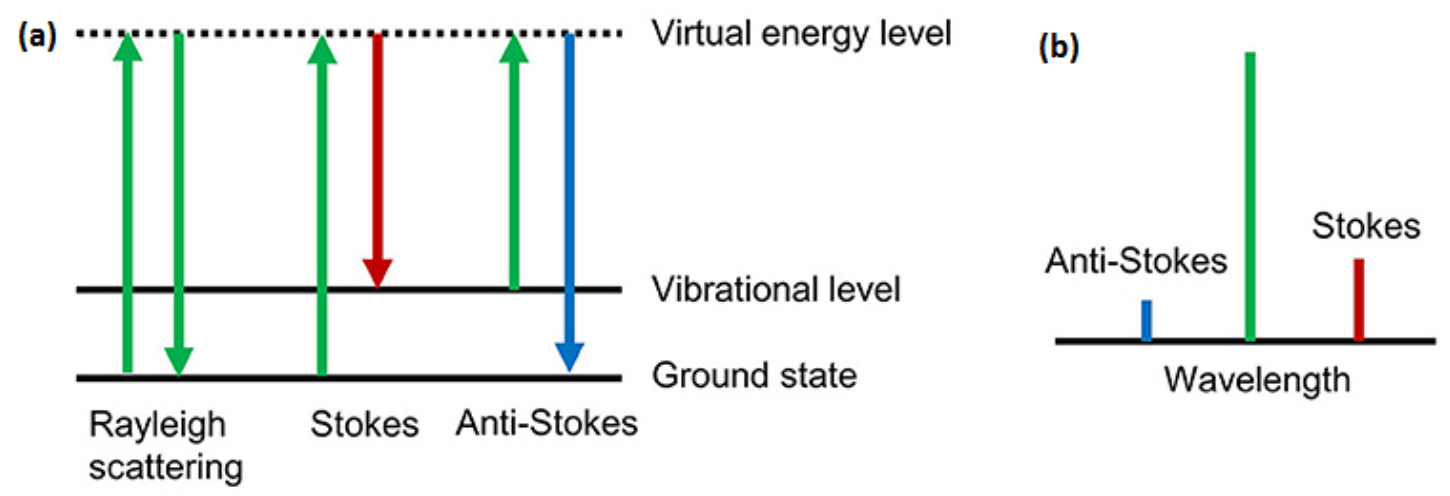

Figure 2.7 (a) Energy-level diagram showing the states involved in Raman spectra. (b) comparison of stokes and anti-strokes scattering with respect of incident wavelength. ${ }^{51}$

When the frequency of incident radiation is higher than the frequency of scattered radiation, Stokes lines appear in the Raman spectrum. But when the frequency of incident radiation is lower than the frequency of scattered radiation, then anti-Stokes lines appear in the Raman spectrum. Stokes Raman bands involve the transitions from lower to higher energy vibrational levels and thus Stokes bands are more intense compared to anti-Stokes 
bands and produce Raman signal in conventional Raman spectroscopy ${ }^{50,52,53}$ as shown in Figure 2.7. Anti-Stokes bands are measured with the fluorescing samples since fluorescence causes the interference with Stokes bands. ${ }^{49}$

C.V. Raman showed that, photons energy that are scattered inelastically serves as a 'fingerprint' for the substance which scatters light. ${ }^{46,47}$ Therefore, Raman spectroscopy is now widely used in the chemical laboratories to identify virtually any kind of material. ${ }^{54}$

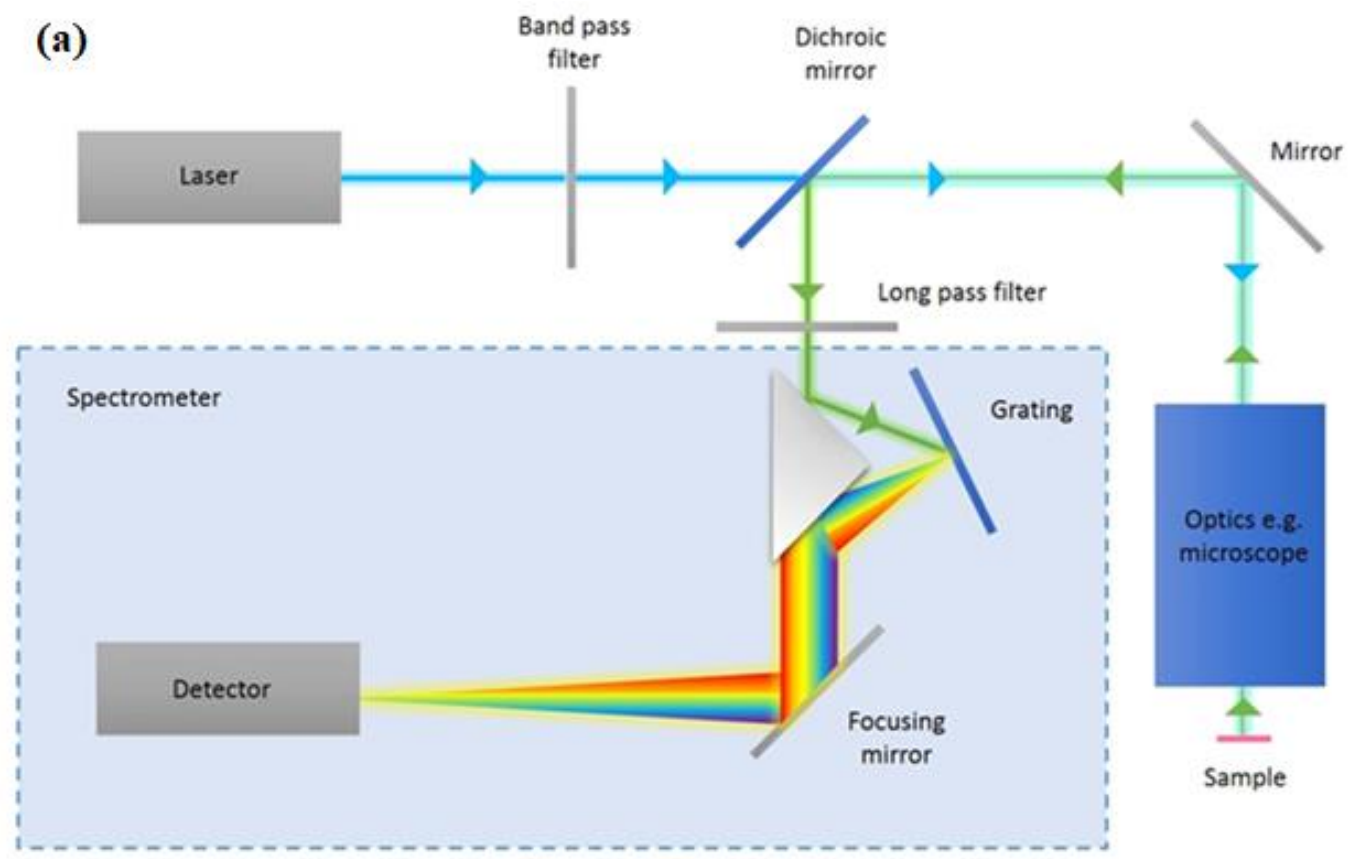

(b)

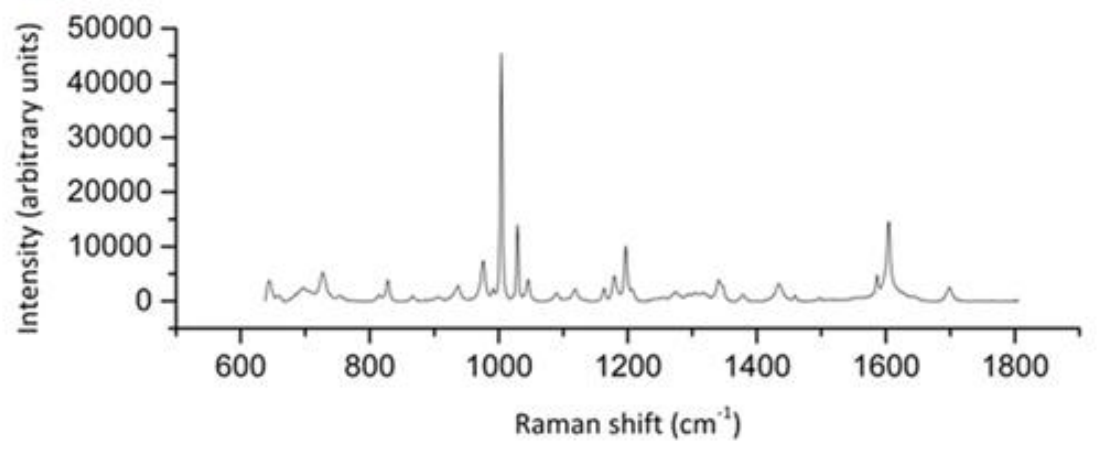

Figure 2.8 (a) Schematic of a Raman spectrometer (b) Raman spectrum of phenylalanine crystals. $^{54}$ 
Schematic diagram of a Raman spectrometer is shown in Figure 2.8 (a). The blue line in Figure 2.8 represents the excitation light while the scattered light is represented by green line. Excitation light travels the path from a laser source to a narrow band pass filters, beam expander and a dichroic mirror as shown in Figure 2.8. Typically, in a Raman spectroscopic measurement the Rayleigh scattering is usually filtered out by either a notch filter, edge pass filter, or a band pass filter, and the rest of the inelastically scattered radiation is dispersed on the detector of Raman spectrometer and produce Raman signal. ${ }^{54}$

A typical Raman spectrum of an analyte is a plot of intensity of scattered light in wavenumbers relative to the incident laser excitation. Here, wavenumber is a variable which is proportional to frequency.

During a typical Raman measurement, specific molecular bonds of a molecule vibrate at particular wavenumbers which corresponds to the characteristic spectroscopic fingerprint of that molecule. ${ }^{54}$ Figure 2.8 (b) shows the Raman spectrum of phenylalanine crystals recorded by a Raman spectrometer excited by a $532 \mathrm{~nm}$ laser with $30 \mathrm{~mW}$ laser power and 1 seconds exposure time using a 50× air objective.

Usually spontaneous Raman scattering is very weak; because of the main difficulty in collecting Raman spectra of weak inelastically scattered light from the intense Rayleigh scattered laser light. ${ }^{46,50}$ Due to this weak Raman signal, it is very difficult to identify molecules through Raman spectroscopy. Therefore, it is important to enhance the naturally weak Raman scattering to produce a prominent Raman signal to detect molecules. Surface enhance Raman scattering (SERS) is a promising tool which can significantly enhance the natural weak Raman signal and has been widely used for the efficient detection of a 
molecule. ${ }^{54,55}$ Basic principle of SERS and sensing method based on promising SERS tool are described in the following section.

\subsubsection{Basic principle of SERS}

SERS is a powerful "fingerprint" technique that offers several orders of magnitude (as high as $10^{14-15}$ ) enhanced Raman signal, which enable even single molecule detecion. ${ }^{13}$

A typical SERS substrate consists of rough metal surface at the nanoscales. For example metallic nanostructures are excellent candidate for SERS detection due to the hot-spots generated on the nanostructures. ${ }^{56-59}$
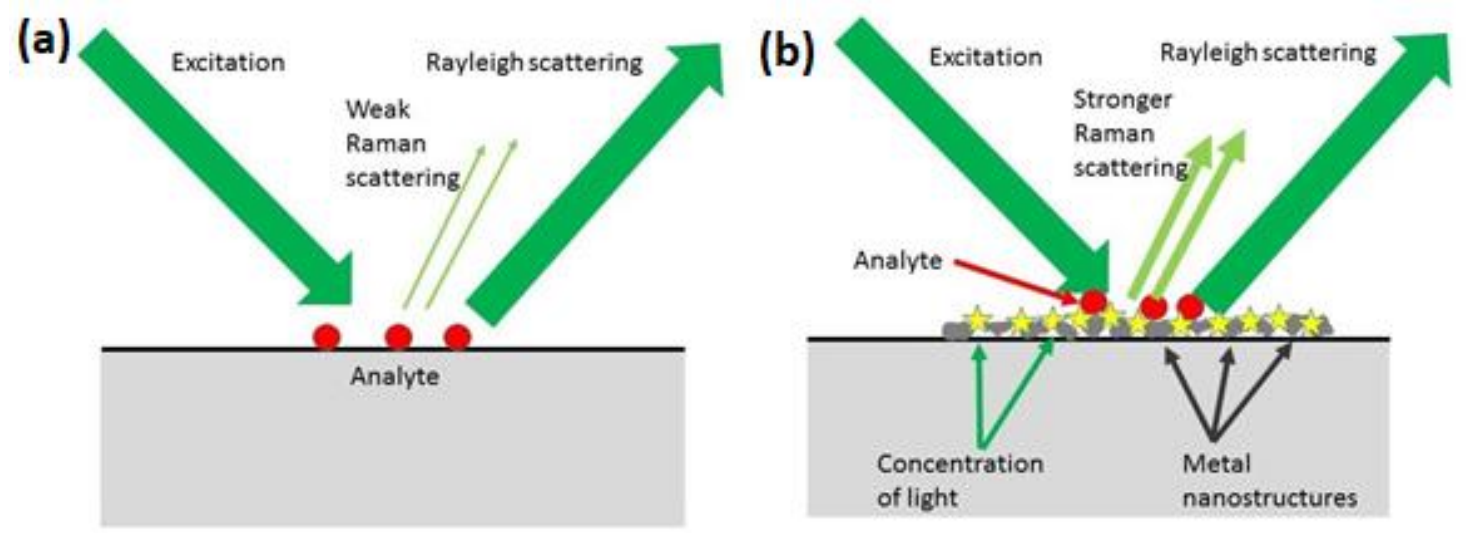

Figure 2.9 (a) Weak Raman scattering without metal nanostructure roughness (without SERS) (b) strong Raman scattering with metal nanostructure roughness (with SERS). ${ }^{17}$

Although the cause of SERS has been a topic of debate, it is accepted that this phenomenon happens due to the combination of a dominant contributor, (EM) electro-magnetic mechanism $^{60}$ and a $(\mathrm{CM})$ chemical mechanism. ${ }^{61} \mathrm{EM}$ mechanism refers to the electron movement in the surface of substrate, while CM involves charge transfer between substrate and target molecules. 
On a particular surfaces the increase in intensity of Raman signal occurs due to an enhancement in the electric field provided by the surface. When incident light strikes the metallic nanostructures, localized surface plasmons are excited on the nanostructures with significantly enhanced electric field. The EM mechanism, which depends on the nanostructures on SERS substrate, dominates the enhanced Raman signal. Roughened surfaces or metallic nanoparticles are typically used in SERS since they provide an enhanced electric field on the surface for enhanced Raman scattering. ${ }^{50}$ Figure 2.9 schematically shows weak and strong Raman scattering without and with metal nanostructures, respectively.

\subsubsection{SERS substrates and SERS measurement}

In a typical SERS measurement, the sample is placed on a substrate known as SERS substrate and SERS measurement is performed by Raman spectroscopy. Figure 2.10 (a) shows the schematic of a typical SERS measurement.
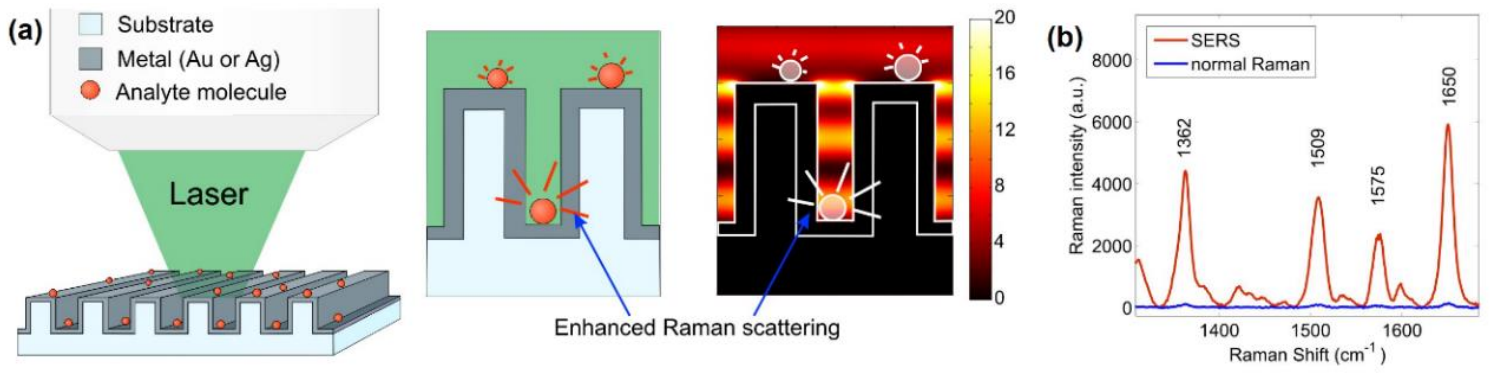

Figure 2.10 (a) Schematic of a SERS measurement; (b) comparison of the detection of an analyte, Rhodamine 6G (R6G) with and without SERS. ${ }^{17}$

Gold, silver and other metals are commonly used in a SERS substrate due to their strong plasmonic resonance. ${ }^{59}$ During the SERS measurement when the light source for example 
laser strikes the analyte attached with metal as schematically shown in Figure 2.10 (a), then the localized surface plasmon resonance of metal is excited along with an enhanced electric field which provide an enhanced Raman scattering as shown in Figure 2.10 (a). Due to this enhanced Raman scattering, SERS is able to significantly enhance the natural weak Raman scattering and can efficiently detect an analyte. For example, $1 \mu \mathrm{M}$ Rhodamine 6G (R6G) is detected through the promising SERS tool as marked by the red curve in Figure 2.10 (b) which is barely detectable with normal Raman measurements of R6G as marked by the blue curve in Figure 2.10 (b).

Most of the SERS substrates reported in the literature are manufactured through either topdown or bottom-up methods, such as focused-ion beam lithography, ${ }^{62}$ electron beam lithography, ${ }^{63}$ nanolithography ${ }^{64}$ as shown in Figure 2.11 (a-c).

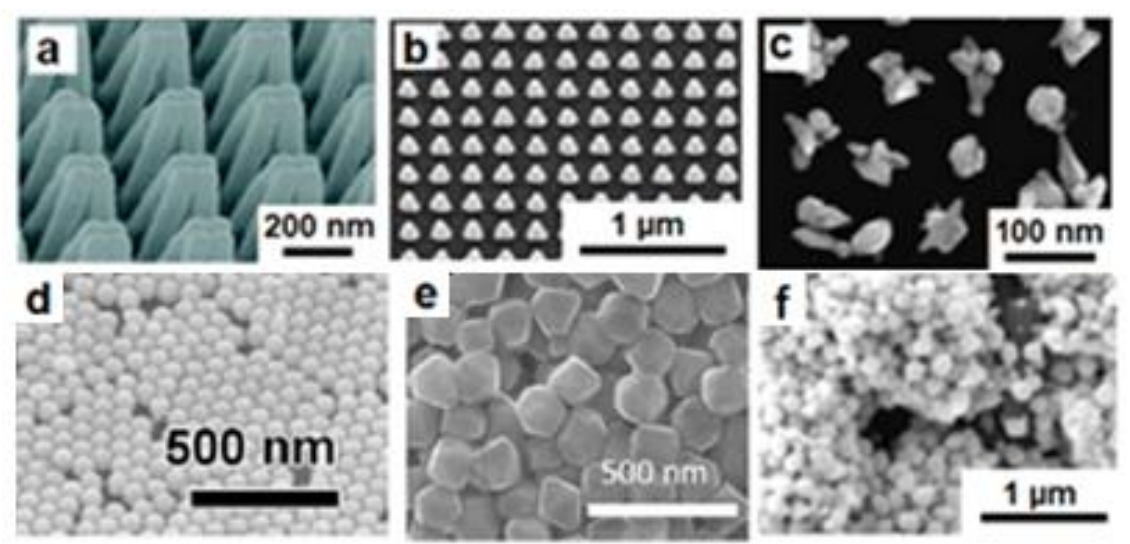

Figure 2.11 SEM image of different types of SERS substrates manufactured by several methods. (a) Au coated polymer nanofingers SERS manufactured by focused ion beam lithography ${ }^{62}$ (b) Au triangular nanoprism SERS manufactured by e-beam lithography ${ }^{63}$ (c) AuNSs SERS by nanolithography ${ }^{64}$ (d) self-assembled gold nanosphere arrays ${ }^{65}$ (e) aluminum nanocrystals ${ }^{66}$ fabricated by chemical synthesis (f) AuNPs SERS by pen-andpaper approach. ${ }^{67}$

A molecular trap structure based on gold-coated nanoscale polymer fingers as shown in Figure 2.11 (a) are formed by focused ion beam lithography ${ }^{62}$ in order to capture analyte 
molecules in solution. Analytes are detected and identified through gold-coated nanoscale polymer fingers SERS substrate. These type of structures can be also used for other applications, such as meta-materials, plasmonics, and other nanophotonic systems. ${ }^{62}$ SERS on an array of gold triangular nanoprisms with precisely controlled spacing and size as depicted in Figure 2.11 (b) has been fabricated through electron beam lithography technique and used for biosensing application. ${ }^{63}$ A large-scale homogeneous gold nanostars SERS substrates as illustrated in Figure 2.11 (c) has been fabricated by the combination of electroless deposition and block-copolymer micelle nanolithography and used for SERS imaging. ${ }^{64}$ All the above-mentioned methods provide excellent outcome, however need time-consuming and tedious fabrication processes.

In order to sovle these problems, several affordable methods as depicted in Figure 2.11 (df) have been developed in recent years. For example, gold nanosphere arrays with rich nanogaps have been fabricated by Liu et al, using self-assembly process ${ }^{65,68,69}$ as shown in Figure 2.11 (d). Nanogaps in this structure provides large SERS enhancement and enables a wide range of application including sensing, catalysis, biology. ${ }^{65,69}$ Another nanogap-rich structure which is used for SERS based DNA detection has been fabricated by chemical synthesis of aluminum nanocrystals ${ }^{66}$ as shown in Figure 2.11 (e). An affordable gold nanoparticles SERS substrate is fabricated by pen-on-paper (POP) approach and used for chemical sensing and pesticide detection ${ }^{67}$ as shown in Figure 2.11 (f).

Several other nanogap-rich structures such as Ag-ring arrays, ${ }^{70}$ 3D-Ag@ZnO nanostructures ${ }^{71}$ split-wedge antenna, ${ }^{72}$ have been fabricated for SERS-based sensing. However, most of these SERS sensors still rely on passive sensing and thus still suffer from the diffusion limit of convention sensing methods. 


\subsection{Active sensing used in this research and questions to be addressed}

In this research, we have demonstrated a cost-effective and time-efficient active sensing method, which not only enables us a rapid fabrication of nanogap-rich-structures under ambient conditions, but also actively guide and locally concentrate analytes on the nanogap-rich structures for active sensing. This method works based on an optothermally generated microbubble (OGMB) - a micron-sized bubble that is generated on a liquid-solid interface through laser heating. The size and position of OGMB can be controlled remotely by a laser. OGMB induces a strong convective flow known as Marangoni convective flow $^{73-78}$ which allows rapid fabrication of nanogap-rich structures for active analytes sensing. More details about OGMB will be discussed in the next chapter. This active sensing method can overcome the diffusion limit of conventional passive sensing method and can be used for advanced chemical and biosensing application. 


\section{CHAPTER 3}

\section{BASIC PRINCIPLE AND APPLICATION OF OGMB}

In this research, we have used optothermally generated microbubble (OGMB) to fabricate nanogap-rich structure which serve as a SERS substrate for active and ultrasensitive chemical and biosensing application. In this chapter, we will discuss the basic principle of an OGMB and its promising application in different fields.

\subsection{Basic principle of OGMB}

Optothermally generated microbubble (OGMB) is a micron-sized bubble that is formed on a liquid-solid interface due to an optothermal effect induced by laser heating. ${ }^{73-76}$ It is a micron sized bubble that stays in contact with a surface, which is different from a floating bubble. Its size and position on a surface can be controlled remotely through a laser beam. Detailed applications of a surface bubble is reported in a recent review by Zhao and Xie. ${ }^{77}$
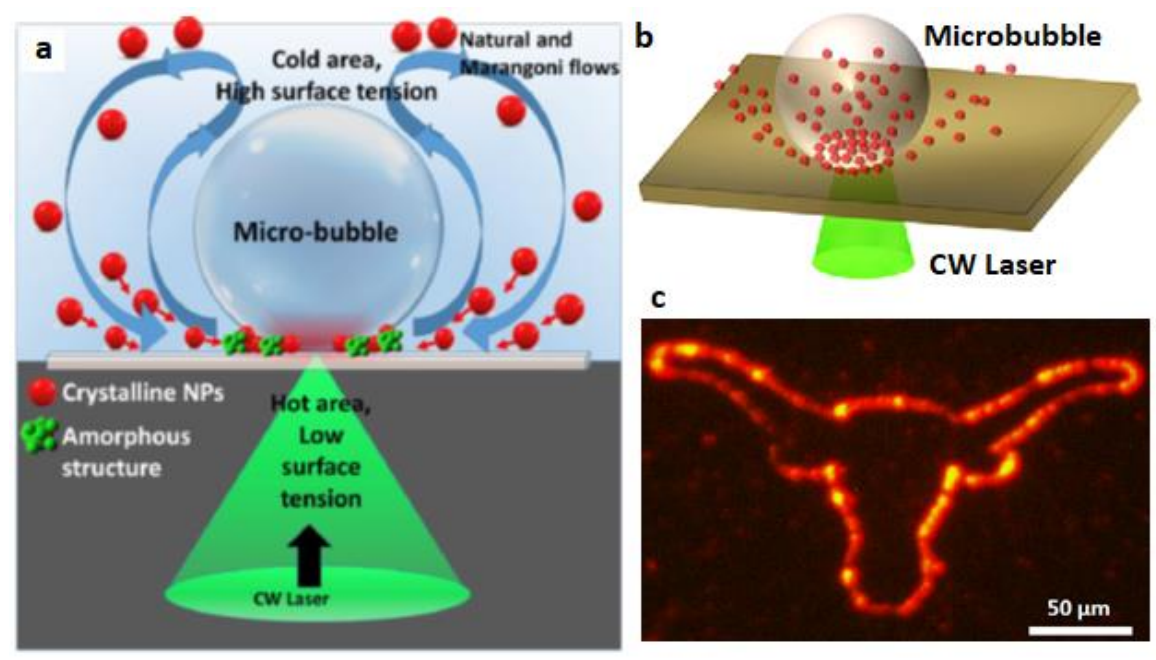

Figure 3.1 (a) Schematic of natural and Marangoni convective flow associated with microbubble generation through the continuous wave $(\mathrm{CW})$ laser heating and rapid deposition of nanoparticles on the surface of substrate due to convective flow ${ }^{79}(\mathrm{~b})$ quantum dots (Q-dots) are attracted towards microbubble because of strong convective flow induced by $\mathrm{CW}$ laser heating ${ }^{80}$ (c) a pattern structure formed through microbubble printing of Qdots. $^{80}$ 
OGMB induced a strong Marangoni convective flow which can be used to deposit nanoparticles ${ }^{74,78,79}$ as shown in Figure 3.1 (a). Figure 3.1 (b) shows the formation of microbubble through a continuous wave (CW) laser heating and quantum dots (Q-dots) are attracted towards microbubble due to strong convective flow. Figure 3.1 (c) depicts a pattern structure formed by microbubble printing of Q-dots.$^{80}$ Fluid convection is divided into two convections known as natural and Marangoni convection. However, contribution of natural convection ${ }^{81,82}$ is much smaller than that from Marangoni convection. Therefore, fluid flow around an OGMB is dominated by strong Marangoni convection.
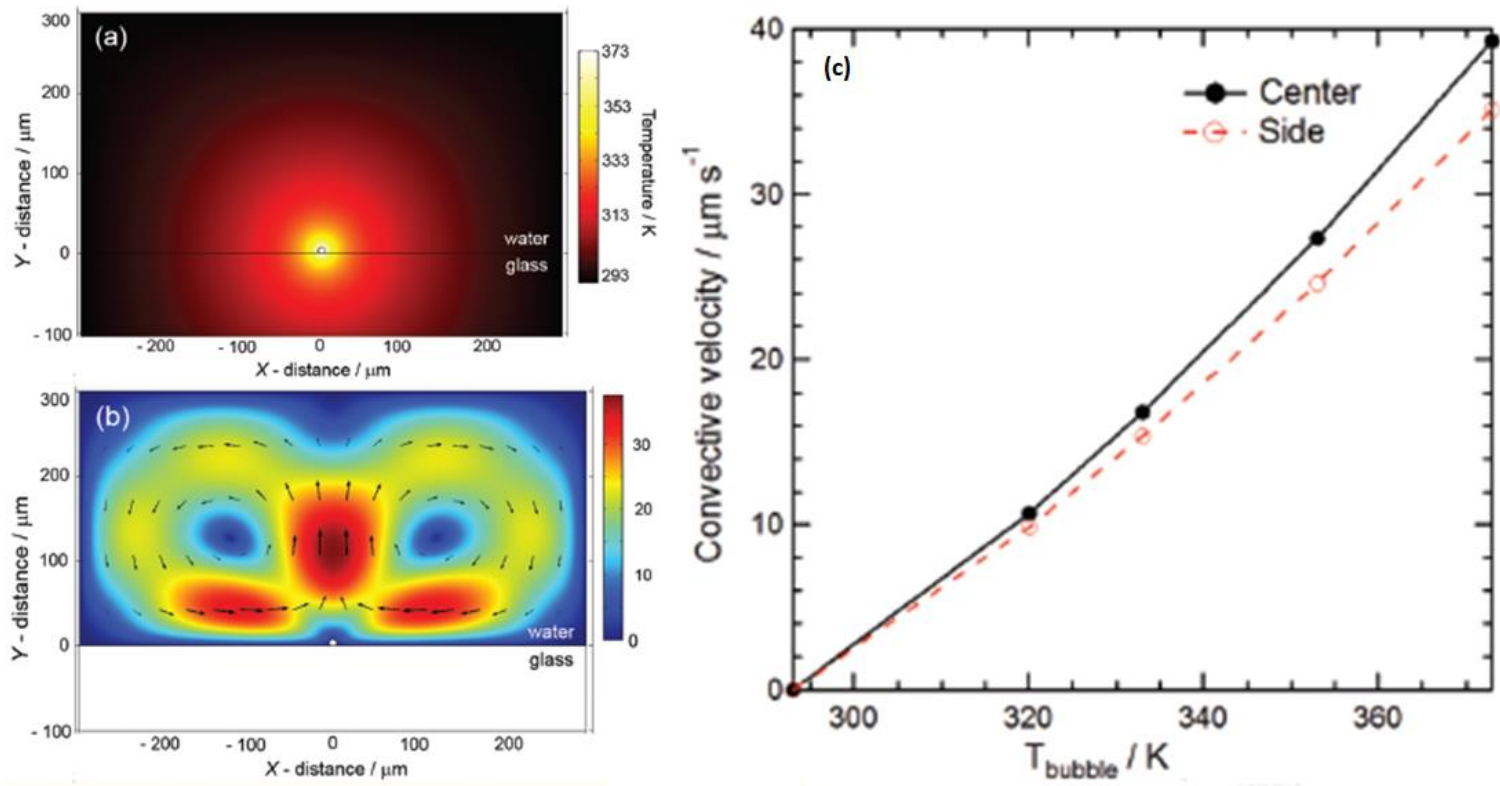

Figure 3.2 (a) 2D temperature distribution and (b) corresponding velocity field around a microbubble at fixed $373 \mathrm{~K}$ temperature (c) convective velocities at the center and side of microbubble as a function of microbubble temperature $\left(\mathrm{T}_{\text {bubble }}\right){ }^{78}$

Figure 3.2 (a) depicts 2D temperature distribution around an OGMB. Figure 3.2 (b) shows the corresponding convective velocity around an OGMB in water. The convective veolociy can reach up to $40 \mu \mathrm{ms}^{-1}$ at the center $(\mathrm{x}$-distance $=0 \mu \mathrm{m} ; \mathrm{y}$-distance $=120 \mu \mathrm{m})$ of the water domain. Figure 3.2 (c) depicts the convective velocities at the center and side of 
microbubble as a function of microbubble temperature ( $\left.\mathrm{T}_{\text {bubble }}\right)$. Convective velocity was almost linear as shown in Figure 3.2 (c) which attributed to the constant thermal expansion coefficient of water. The following properties of microbubble and Marangoni convection induced by microbubble under $\mathrm{CW}$ laser heating was reported $^{78}$ in the literature: (i) diameter of the microbubble increases with the increase in laser power density; (ii) no strong convective flow is obserabale without the formation of a microbubble; (iii) highest temperature was observed at the center of the microbubble; (iv) contribution of natural convection was minor due to lower temperatures at the surface of microbubble; (v) effective convection was observed around $100 \mu \mathrm{m}$ radial distance from the center of microbubble; (vi) as soon as the diameter of microbubble exceeded a certain size, convective velocity gradually saturated due to the gradual saturation in temperature at the surface of microbubble.

\subsection{Application of OGMB}

OGMB induces a strong Marangoni convective flow which can be used for rapid deposition of nanoparticles and develop several pattern structures. For example, Zheng et al. demonstrated the deposition of $1-\mu \mathrm{m}$ polystyrene particles on silver film ${ }^{83}$ through an OGMB. Figure $3.3(\mathrm{a}-\mathrm{c})$ illustrates polystyrene particles are attracted towards microbubble due to strong convective flow. The schematic of convective flow and particles accumulation due to convective flow is depicted in Figure 3.3 (d). Though the deposition of polystyrene particles on silver film three types of pattern structures, ring, square lattice and swiss roll have been developed as depicted in Figure 3.3 (e-g). 

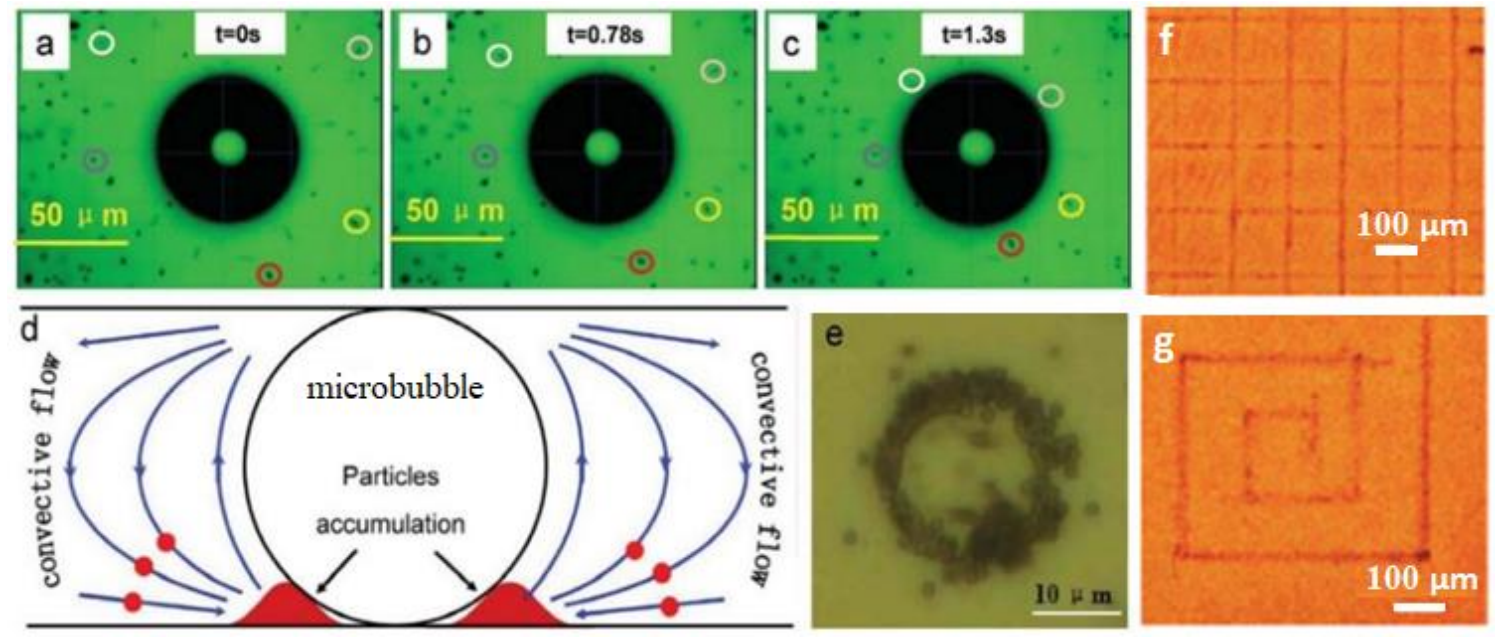

Figure 3.3 (a-c) Temporal distribution of microbubble-generation (d) schematic of microbubble generation and convective flow pattern (e-g) three types of pattern structures; (e) ring (f) square lattice (g) swiss roll developed through optothermal microbubble generation. ${ }^{83}$

A "bubble-pen-lithography (BPL)" is developed by Zheng and co-workers ${ }^{84}$ to achieve patterning of colloidal particles on a gold nanoislands (AuNIs) substrate by optically controlled microbubbles as schematically shown in Figure 3.4 (a). Colloidal particles are driven towards the microbubble through Marangoni convection induced by the microbubble. The simulated profile of Marangoni convection around the microbubble is shown in Figure 3.4 (b). Due to the convective flow colloidal particles are rapidly deposited on AuNIs substrate. The diameter of microbubble and size of printed colloidal particles is precisely controlled by tuning optical power density as illustrated in Figure 3.4 (c, d). BPL is used for patterning single particles into different arbitrary patterns as shown in Figure 3.4 (e-h). A 'Mona-Lisa' pattern consists of semiconductor QDs has been printed with this method as depicts in Figure 3.4 (i-k). Figure 3.4 (1) shows a map of U.S. with the states of Texas, California, and Pennsylvania printed with different QDs fabricated through a multistep printing process. 

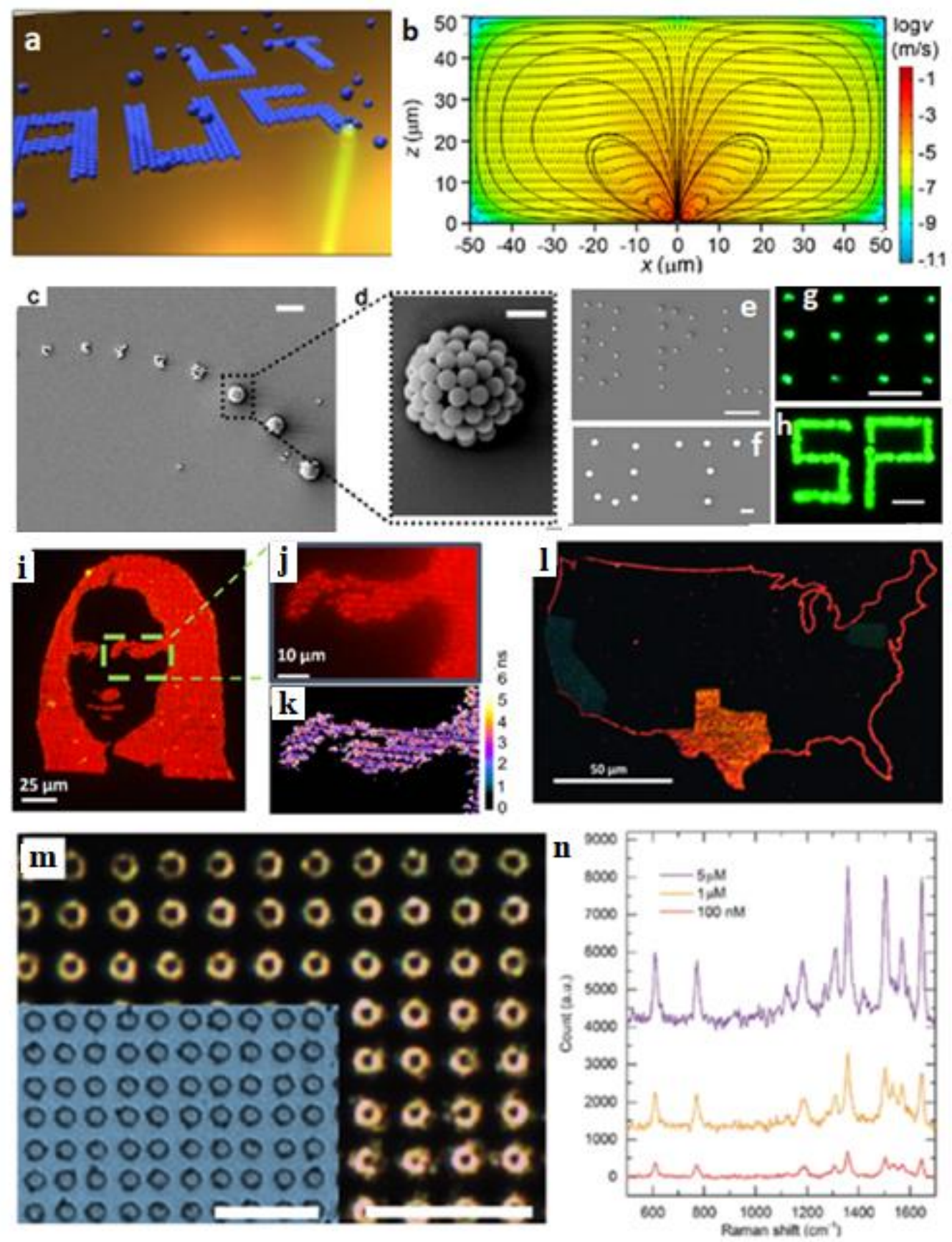

Figure 3.4 (a) Schematic illustration of Bubble-Pen-Lithography $(B P L)^{84}$ (b) simulated profile of Marangoni convection around a $1 \mu \mathrm{m}$ microbubble $^{84}$ (c,d) SEM image of (c) patterned PS beads at different laser intensity; scale bar $5 \mu \mathrm{m}$ (d) 3-D structure of aggregated PS beads; scale bar $1 \mu \mathrm{m}$ (e) "BPL" and (f) "UT" pattern from PS beads ( $\mathrm{g}, \mathrm{h}$ ) dark-field optical image of (g) $4 \times 4$ array of 3D hollow structures and (h) "SP" pattern of PS beads ${ }^{84}$; scale bar in (e-h) $10 \mu \mathrm{m}$ (i-k) "Mona Lisa" pattern of red QDs with fluorescence lifetime mapping ${ }^{85}$ (1) fluorescence image of U.S. map depicting states of Texas, Pennsylvania, California printed with different $\mathrm{QDs}^{85}(\mathrm{~m})$ bright-field and dark-field optical image of an Ag ring array fabricated through BPL; scale bar $10 \mu \mathrm{m}$ (n) SERS spectra of different concentration of R6G collected by Au ring array on AuNIs substrate. ${ }^{70}$ 
Besides the printing of colloidal particles, BPL also demonstrated the fabrication of metallic nanostructures through the printing of $\mathrm{Ag}$ rings array on gold nano-islands ( $\mathrm{Ag}$ ring on AuNIs) as shown in Figure 3.4 (m). SERS spectra of different concentration of Rhodamine 6G (R6G) are detected by Ag ring on AuNIs as shown in Figure 3.4 (n).
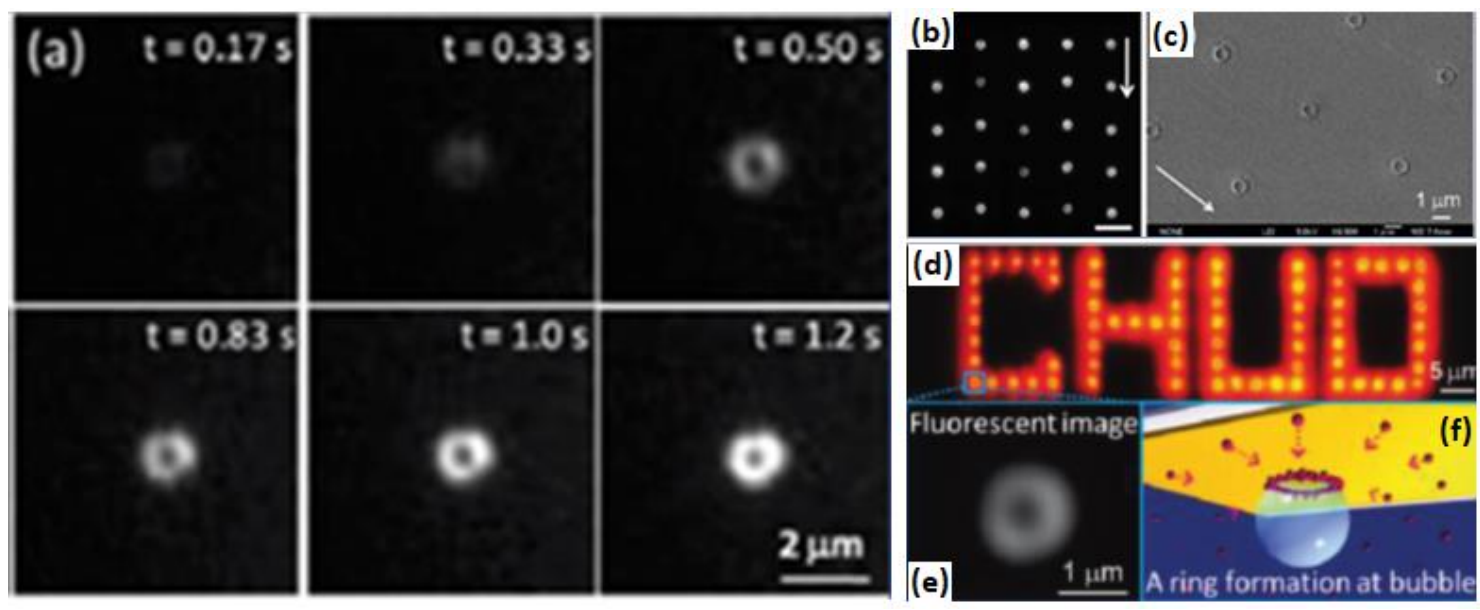

Figure 3.5 (a) Temporal distribution of microbubble generation from Q-dots (b) microscope image of ring array fabricated from Q-dots; scale bar $5 \mu \mathrm{m}$ (c) SEM image of fabricated ring array of Q-dots on a gold film ${ }^{86}$ (d) pattern structure of Q-dots (e) magnified image of part (d) marked by a box (f) schematic of a ring formation through microbubble generation on gold film. ${ }^{86}$

Fujii et al. fabricated ring structure through the deposition of quantum dots (Q-dots) on a gold film ${ }^{86}$ using OGMB. Figure 3.5 (a) illustrates the fluorescent images of the temporal change of ring formation of CdSe Q-dots by focusing laser on the surface of gold film. Fluorescence microscope image of a ring array composed of CdSe Q-dots on gold surface is depicted in Figure 3.5 (b) and the corresponding SEM image of the ring array is shown in Figure 3.5 (c). A pattern structure of Q-dots is depicted in Figure 3.5 (d) and the magnified image of a part of Figure 3.5 (d) which is marked by a box is shown in Figure 3.5 (e). The schematic of ring formation through microbubble generation on gold film ${ }^{86}$ is shown in Figure 3.5 (f). 

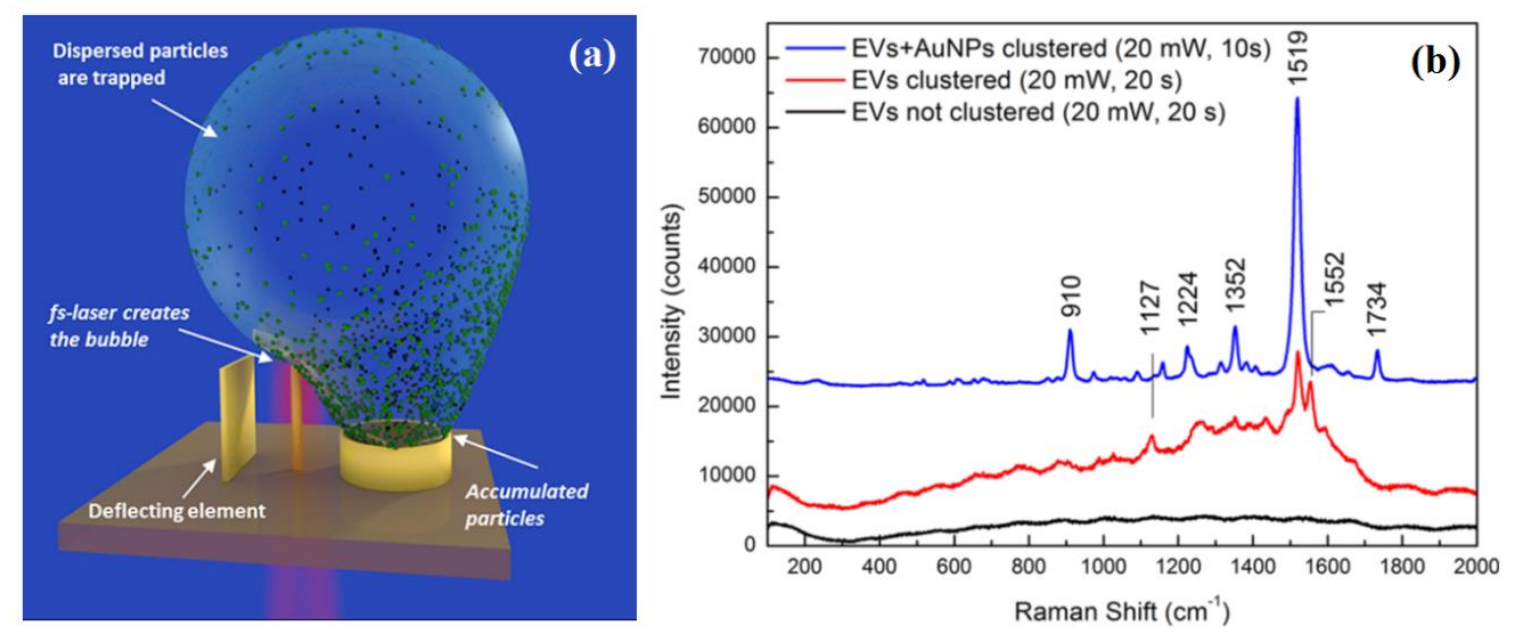

Figure 3.6 (a) Controlled trapping and accumulation of EVs towards the sensor surface through OGMB. (b) Raman spectra of EVs collected at different condition; EVs clustered (red line), EVs not clustered (black line), EVs with AuNPs clustered (red line) ${ }^{87}$.

Tantussi et. al. demonstrated a method for controlled trapping and delivery of nanoscale objects through an $\mathrm{OGMB}^{87}$ as shown in Figure 3.6. In this work, OGMB was formed on a 3D nanostructured substrate with the help of plasmonic and fluid-dynamic functionalities. A near infrared femtosecond pulsed source was used which minimized the presence of thermal effects and thus enabled the application of this technique for the detection of a biological molecule known as extracellular membrane vehicles (EVs) which are wellknown to be difficult to handle. The concentration of EVs around OGMB is depicted in Figure 3.6 (a) and detection of EVs are shown in Figure 3.6 (b).

Marangoni convective flow ${ }^{73-78}$ induced by OGMB can also be used for many other applications for example plasmo fluidic lenses, ${ }^{88}$ particle trapping,${ }^{74}$ photothermal motors,${ }^{89}$ microfluidic applications, ${ }^{90}$ and so on. However, in all of the above-mentioned methods a special absorptive layer is needed that limits the types of substrate which is used during the generation of OGMB. 
In this work, OGMB can be generated on any kind of substrates under ambient condition. Therefore, there is no limitation on the type of substrate. We have demonstrated OGMBbased rapid fabrication of nanogap-rich structures for active and ultrasensitive chemical and bio-sensing application. This work is different from the works presented in the references $^{70,87}$ as follows: in this work OGMB is not only used for rapid fabrication of nanogap-rich structure under ambient conditions, which eventually serve as a SERS substrate, but also used to concentrate analytes around nanogap-rich structure for active sensing. In reference ${ }^{70} \mathrm{OGMB}$ is only used to fabricate a $\mathrm{Ag}$ ring array which serve as a SERS substrate and eventually used for diffusion limited passive chemical sensing application. In contrast, we have demonstrated active sensing with the help of OGMBbased SERS. In our work, OGMB is used both for the fabrication of SERS substrate as well as concentrate analytes around SERS substrate for active sensing.

The concept of OGMB-based active biosensing is reported in reference. ${ }^{87}$ However, conventional clean-room based methods are still required to pattern the substrate used in reference ${ }^{87}$ for biosensing application. In addition, an expensive femtosecond laser is used to generate OGMB ${ }^{87}$ On the other hand, we have used a low-cost continuous-wave laser to generate OGMB and rapidly fabricate nanogap-rich structure which serve as a SERS substrate. The fabrication of SERS substrate based on an OGMB in this research was conducted under ambient conditions without using expensive clean-room facilities which made our method affordable. The detailed fabrication process of nanogap-rich structure will be discussed in the next chapter. 


\section{CHAPTER 4}

\section{FABRICATION OF NANOGAP-RICH STRUCTURES}

In this research, a nanogap-rich structure has been fabricated through optothermally generated microbubble (OGMB) inside a droplet of metallic nanoparticles solution. The fabricated nanogap-rich structure serves as a SERS substrate due to the plasmonic resonance ${ }^{24-26}$ of nanogap-rich structure. In this chapter, we will describe a cost-effective and rapid fabrication of nanogap-rich structure based on an OGMB, which eventually serves as a SERS substrate. The step-by-step procedure for the fabrication of nanogap-rich structure is described in detail in the following section.

\subsection{Substrate preparation}

In this experiment, a glass coverslip with dimension $22 \times 22 \times 0.19 \mathrm{~mm}$ is used as a substrate. Then, $10 \mathrm{~nm}$ thin layer of gold is coated on the glass coverslip through sputtering technique using Denton desk II sputter coater. Sputtering process is performed under vacuum environment at $50 \mathrm{mTorr}$ pressure and $45 \mathrm{~mA}$ current for 30 seconds and developed a smooth layer of $10 \mathrm{~nm}$ gold thin film on glass coverslip. It should be mentioned that, the deposition of a gold film is not a prerequisite in our experiment. The thin layer of gold film is used to enhance the optothermal effect during the generation of OGMB and also facilitate the imaging with a scanning electron microscope (SEM) as described in one of our publications. ${ }^{22}$ Glass coverslip without gold film is also used as a substrate in this work for comparsion. However, a higher laser intensity is needed to generate microbubble on a glass coverslip without gold film. Figure 4.1 shows the schematic of two types of 
substrates (glass coverslip with and witout gold film) which are used for the fabrication of nanogap-rich structure.
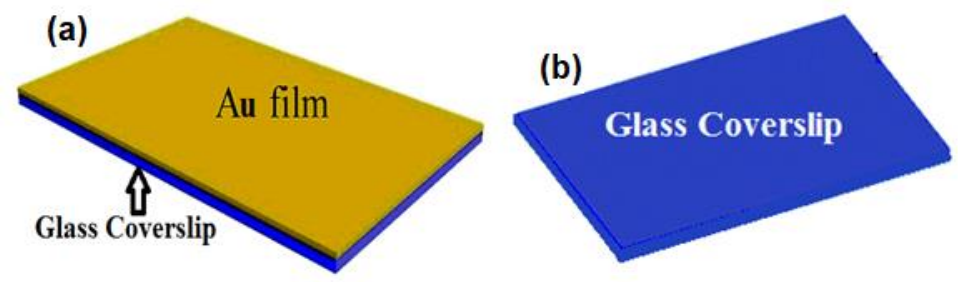

Figure 4.1 Schematic of (a) glass coverslip coated with $10 \mathrm{~nm}$ gold film (b) glass cover slip without any gold coating.

\subsection{Develop optical setup}

A near infrared continuous wave laser of wavelength, $\lambda=1064 \mathrm{~nm}$ was used as a heating laser and focused on the substrate through an objective lens $\mathrm{O}_{1},(N A=0.65,40 \times)$ in our experiment. Figure 4.2 shows the schematic of experimental setup for optothermal microbubble generation. As shown in Figure 4.2, laser beam was expanded as well as collimated by a beam expander through the combination of two lenses, $\mathrm{L}_{1}$ and $\mathrm{L}_{2}$ to overfill the back aperture of objective lenses $\mathrm{O}_{1}$.
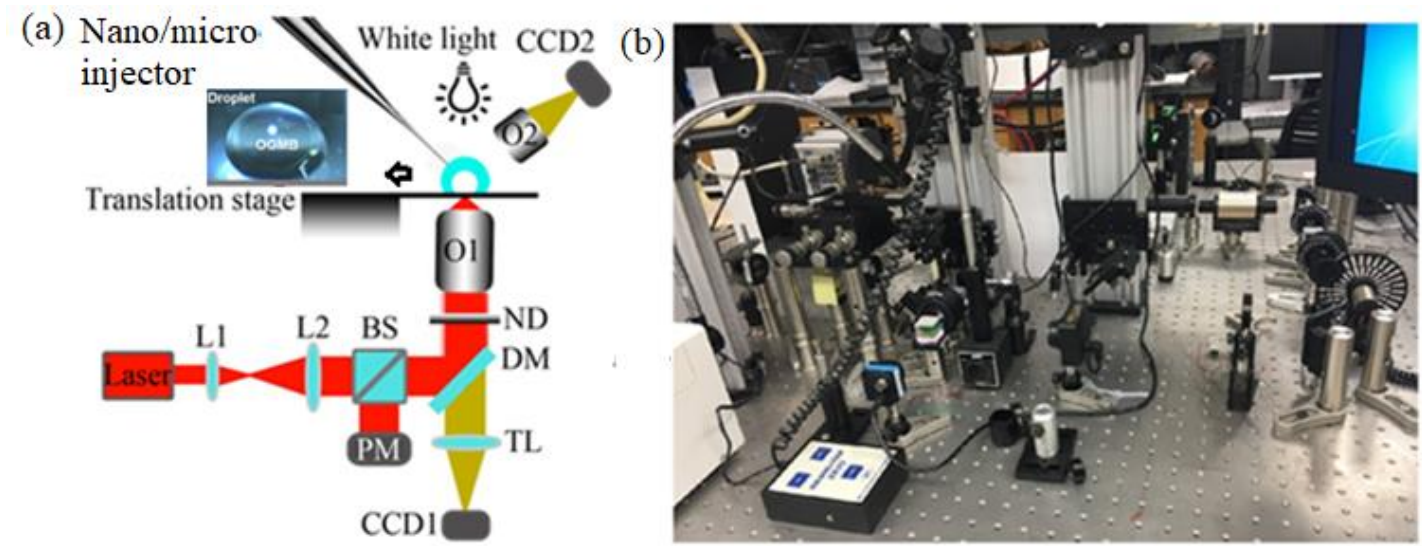

Figure 4.2 (a) Schematic of experimental setup for optothermal microbubble generation. ${ }^{22}$ L: lens; BS: beam splitter; DM: dichroic mirror; O: objective lens; TL: tube lens; CCD: charge coupled device; ND: variable neutral density filter; PM: power meter. Inset: optical image of an OGMB generated inside the droplet of nanoparticles solution (b) real experimental setup. 
The laser beam was focused on the sample through the microscope objective lens, $\mathrm{O}_{1}$. A variable neutral density filter (ND) was used to control the intensity of laser beam. The laser beam was splitted by a beam splitter and fraction of laser power was picked up by a power meter. The relation between the laser power recorded on the power meter and the power focused on the substrate were calibrated in such a way that, power at the substrate can be immediately calculated from the measured power on the power meter. A white-light source is use to illuminate the substrate. The substrate was then imaged on a chargecoupled device $\left(\mathrm{CCD}_{1}\right)$ through microscope ojective lens, $\mathrm{O}_{1}$ and a tube lens (TL). The purpose of using dichroic mirror (DM) in the optical setup was to reflect infrared laser beam but transmit white light. A micro/nano injector was used to generate one droplet of mettalic nanoparticles solution on the substrate. The substrate was mounted on a 3-axis translation stage (Thor lab Nano Max 300) for controlling the precise motion of the substrate. Another imaging system, that consists of a charge-coupled device $\left(\mathrm{CCD}_{2}\right)$ and a long-working-distance objective lens $\left(\mathrm{O}_{2}\right)$ was mounted on a cage system at an angle of $45^{\circ}$ to the optical axis. The side-view of the nanoparticles droplet on the substrate can be imaged on the charge-coupled device $\left(\mathrm{CCD}_{2}\right)$.

\subsection{Microbubble generation through laser heating}

Microliter pipette and nanoliter injector were used to generate micron and nano-liter size droplet of metallic nanoparticles solution respectively. After generating droplet of nanoparticles solution at the desired location on the substrate, it was then heated by the focused laser beam as schematically shown in Figure 4.3 (a). A near-infrared continuouswave laser of wavelength $1064 \mathrm{~nm}$ was used as a heating laser which was focused on the 
substrate with a beam waist of around $0.7 \mu \mathrm{m}$. The laser-based heating can deliver a high energy density to a small confined area for localized heating of nanoparticles solution which creates an optothermal effect. Due to this optothermal effect a micron-sized bubble was generated inside the droplet of metallic nanoparticles solution which is known as optothermally generated microbubble (OGMB) as shown in Figure 4.3 (b).
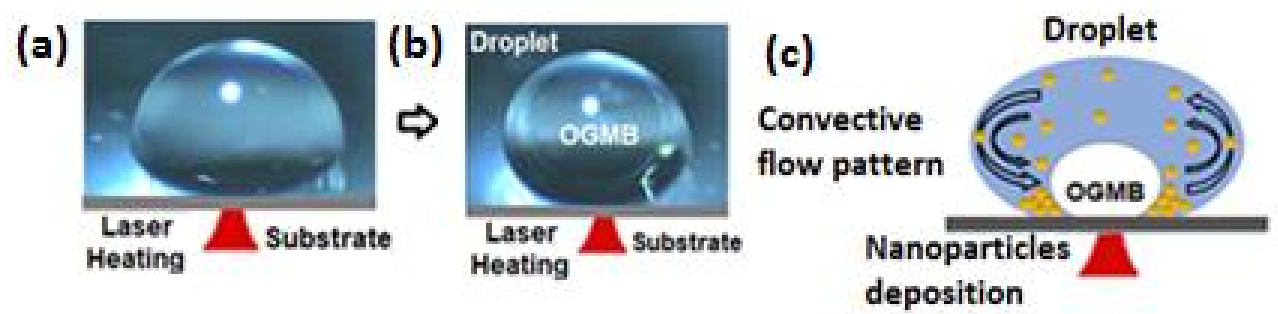

Figure 4.3 (a) Optical image of a nanoliter droplet heated by laser beam (b) an OGMB generated inside a nanoliter droplet of nanoparticles solution. ${ }^{22}$ (c) schematic of optothermal microbubble generation and nanoparticles deposition on the substrate due to convective flow induced by OGMB

OGMB creates a strong convective flow by which nanoparticles are attracted towards the microbubble and deposited on the surface of the glass substrate. The working principle of microbubble generation and rapid deposition of nanoparticles on the substrate through strong convective flow induced by OGMB is illustrated in Figure 4.3 (c).

\subsection{Fabrication of nanogap-rich structure through OGMB}

In this research, metallic nanoparticles are used to fabricate nanogap-rich structures. Metallic nanoparticles play two roles in this work: (1) act as building blocks for the fabrication of nanogap-rich structures; (2) serve as nano heating sources to generate the optothermal microbubble. In this work, three types of metallic nanoparticles (200 nm goldnanospheres, $240 \mathrm{~nm}$ gold-nanoshells, and $100 \mathrm{~nm}$ gold-nanostars) are used to fabricate 
three-types of nanogap-rich structures. The fabrication processes of three-types of nanogap-rich structures are described in detail in the following section.

\subsubsection{Fabrication of gold-nanosphere-structure}

$200 \mathrm{~nm}$ gold nanosphere solution from BBI Solutions was used in this experiment. Gold nanospheres were used as received without further modification. Nanogap-rich structures were fabricated from both nanoliter size droplet and microliter size droplet of gold nanospheres. In order to fabricate nanogap-rich structure from a nanoliter droplet of goldnanosphere, a nanoliter injector known as 'Nanoliter 2010' from World Precision Instruments as shown in Figure 4.4 (c) was used to generate a droplet of gold nanosphere on a gold-coated glass substrate.

Before generating a nanoliter size droplet of gold nanosphere solution, glass micropipette of nanoliter injector was at first filled with mineral oil. Then glass micropipette of nanoliter injector was filled with gold nanosphere solution. This procedure was performed to ensure consumption of minimum samples and avoid contamination of nano-liter injector. Following the procedure as described above, a droplet of gold nanosphere solution with 276 nanoliter $(\mathrm{nL})$ volume was injected on the gold-coated glass substrate. The heating laser was then focused at the center of liquid-solid (substrate) interface. Some gold nanospheres were settled down on the substrate and were heated by the focused heating laser which therefore generated an optothermal microbubble inside the nanoliter droplet of gold nanosphere as shown in Figure 4.3 (c).

Figures 4.4 (a) and Figures 4.4 (b) demonstrate the optical image of a nanoliter droplet of gold nanosphere before and after the generation of a microbubble, respectively. According 
to these figures, the size of nanoliter droplet increases from $942 \mu \mathrm{m}$ to $954 \mu \mathrm{m}$ in order to occupy the generated microbubble inside the nanoliter droplet of gold nanosphere.
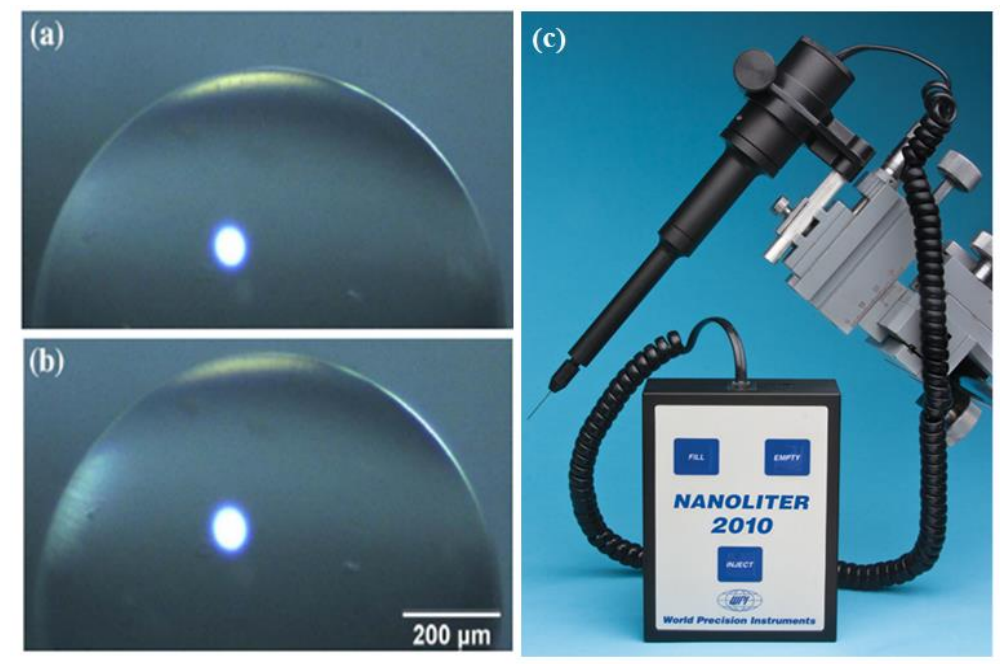

Figure 4.4 Optical image of a nanoliter droplet of gold nanosphere solution (a) before and (b) after generation of an optothermal microbubble inside the droplet of gold nanosphere. ${ }^{22}$ (c) a nanoliter injector known as 'Nanoliter 2010' which was used to create nanoliter droplet of gold nanosphere.

The focused laser beam increases the local temperature of the gold-coated glass substrate and therefore an OGMB is generated inside the droplet of gold nanosphere accociated with a strong Marangoni convective flow around the OGMB. A laser intensity of $75 \mathrm{~mW} / \mu \mathrm{m}^{2}$ was used to generate the OGMB. The strong Marangoni flow induced by the OGMB helps to rapidly deposit gold nanospheres on the gold-coated glass substrate and formed a ringshaped structure. The schematic of convective flow pattern induced by an OGMB is shown in Figure 4.5 (a). Figure 4.5 (b) shows an optical image of a structure that is deposited at the center of the nanoliter droplet of gold nanosphere on the substrate. A ring-shaped structure with a diameter of $37 \pm 0.7 \mu \mathrm{m}$ that consists of gold nanospheres was developed on the substrate. 
(a)

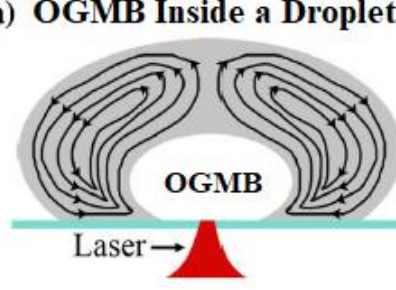

(d)

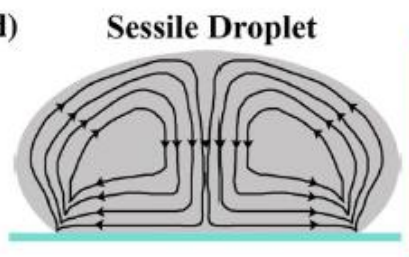

(b)

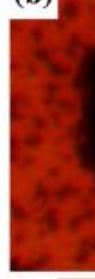

(e)
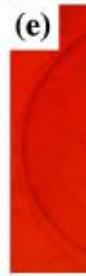

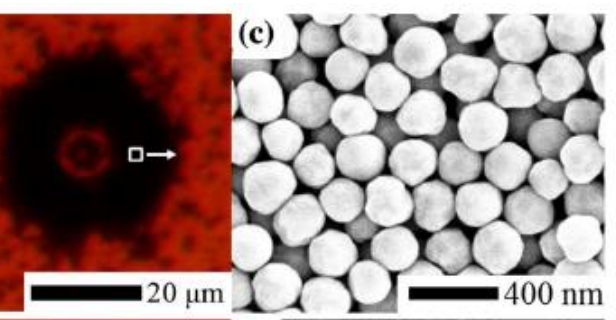

(f)

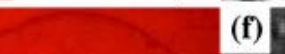

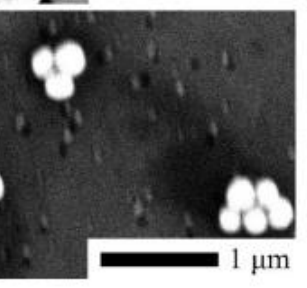

Figure 4.5 (a) Schematic of convective flow pattern induced by an OGMB formed inside a droplet. (b) optical image of a nanogap-rich structures fabricated from a nanoliter droplet of $200 \mathrm{~nm}$ gold nanospheres on a gold-coated glass substrate (c) SEM image of the corresponding area which is marked in (b) by a white box. (d) schematic of radial flow pattern induced by natural evaporation of a sessile droplet of gold nanosphere. (e) optical image of the pattern developed on the same substrate due to the coffee-ring effect induced by the natural evaporation of a sessile droplet of gold nanosphere. (f) SEM image of the corresponding area which is marked in (e) by a white box. ${ }^{22}$

Figure 4.5 (c) shows the SEM image of the corresponding area marked by a white box in Figure 4.5 (b) of the ring-shaped structure. Gold nanospheres accumulated on the substrate and forms a nanogap-rich structure, which is named as gold-nanosphere-structure in this work. The purpose of OGMB is to rapidly deposit nanoparticles on the glass substrate through the strong Marangoni convective flow induced by the OGMB. These experimental results are reported in one of our publications. ${ }^{22}$

It should be noted that, we can also deposit nanoparticles on a substrate by natural evaporation of a sessile droplet of gold nanosphere solution. However, through the natural evaporation of a sessile droplet, nanoparticles will be deposited at the edge of the droplet, not in the center. ${ }^{91-95}$ In order to prove this concept, a sessile droplet of gold nanosphere solution of $276 \mathrm{~nL}$ volume was injected on the same gold-coated glass coverslip substrate 
and then let it evaporate naturally. The primary radial flow inside a droplet induced by the natural evaporation of a droplet ${ }^{96}$ is schematically shown in Figure 4.5 (d). The radial flow induced by the natural evaporation of a nanoparticles droplet usually carries the nanoparticles to the edge of the droplet and develops a ring-shaped structure which is known as the "coffee-ring effect" ${ }^{97}$ The "coffee-ring effect" is formed due to the natural evaporation of a gold nanosphere droplet which is seen in the optical image of a structure as shown in Figure 4.5 (e). Due to this coffee-ring effect, only a few gold nanospheres are deposited in the center area of the droplet as depicted in the SEM image of Figure 4.5 (f). It is reported that, engineering thermal conductivity of the substrate ${ }^{95}$ or adding surfactant ${ }^{94}$ could reverse the direction of radial flow inside an evaporating sessile droplet. This reverse directed radial flow could bring the nanoparticles in the center of the sessile droplet. However, all these approaches may limit the type of substrate or contaminate the sample. Through an OGMB a nanogap-rich structure as shown in Figure 4.5 (b) can be rapidly formed in the center of a droplet. The size of nanogap-rich structure fabricated through an OGMB can be controlled by changing the intensity of heating laser on the substrate, which will be described later in this chapter.

Similar to a nanoliter droplet generated from 'Nanoliter 2010' as described above, microliter droplet of $200 \mathrm{~nm}$ gold nanosphere solution was also used to generate microbubble. Microliter pipette that can carry $5 \mu \mathrm{L}$ volume of solution was used to create a microliter droplet of $200 \mathrm{~nm}$ gold nanosphere. The transmission electron microscope (TEM) image of $200 \mathrm{~nm}$ gold nanosphere is shown in Figure 4.6 (a). The optical images of a gold-nanosphere-structure fabricated from a microliter droplet of $200 \mathrm{~nm}$ gold 
nanosphere is shown in Figure 4.6 (b). These is a ring-shaped pattern structure having diameters of $97 \pm 1 \mu \mathrm{m}$.
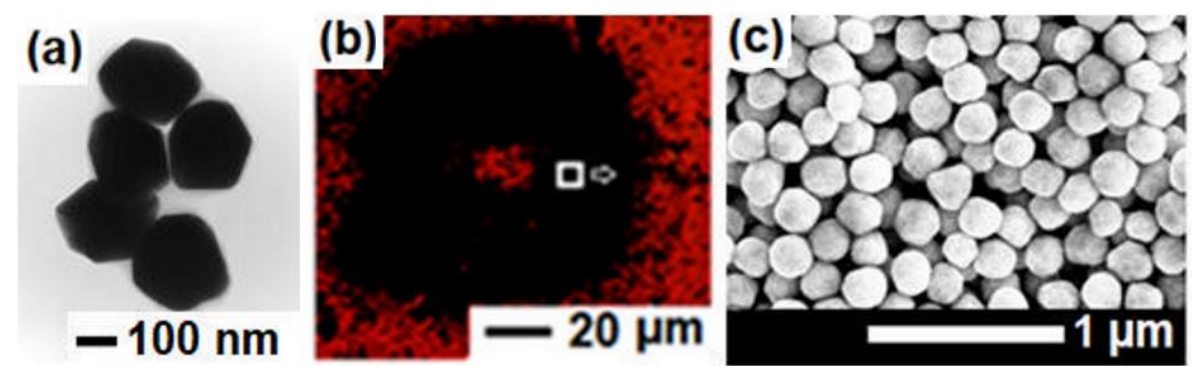

Figure 4.6 (a) TEM image of $200 \mathrm{~nm}$ gold-nanospheres (b) optical image of a nanogap rich structure fabricated from a microliter droplet of $200 \mathrm{~nm}$ gold nanospheres (c) SEM image of the corresponding area of nanogap-rich structure marked in (b) by a white box. ${ }^{23}$

Figure 4.6 (c) depicts the scanning electron microscope (SEM) images of a fabricated goldnanosphere-structure corresponding to the area marked by a white box in Figure 4.6 (b). The gold nanospheres in the nanogap-rich structures form many nanogaps, which are ideal for SERS detection because of the strong plasmonic resonance present in the nanogap-rich structures. ${ }^{24-26}$ Therefore, the nanogap-rich structure fabricated through an OGMB serve as a SERS substrate.

\subsubsection{Fabrication of gold-nanoshell-structure}

$240 \mathrm{~nm}$ gold nanoshell (silica core) was used in this experiment to fabricate another nanogap-rich structure. $240 \mathrm{~nm}$ gold nanoshell (silica core) consists of a $200 \mathrm{~nm}$ silica core coated with $20 \mathrm{~nm}$ gold nanospheres as depicted in the TEM image of Figure 4.7 (a) was purchased from 'NanoComposix'. Gold nanoshells (silica core) was used as received without further modification. Nanogap-rich structures fabricated from gold nanoshells (silica core) are referred in this work as gold-nanoshell-structure. Optical images of a gold- 
nanoshell-structure with diameters of $102 \pm 1 \mu \mathrm{m}$ is shown in Figure 4.7 (b). Figure 4.7 (c) depicts scanning electron microscope (SEM) image of the corresponding area of goldnanoshell-structure which is marked by a white box in Figure 4.7 (b).
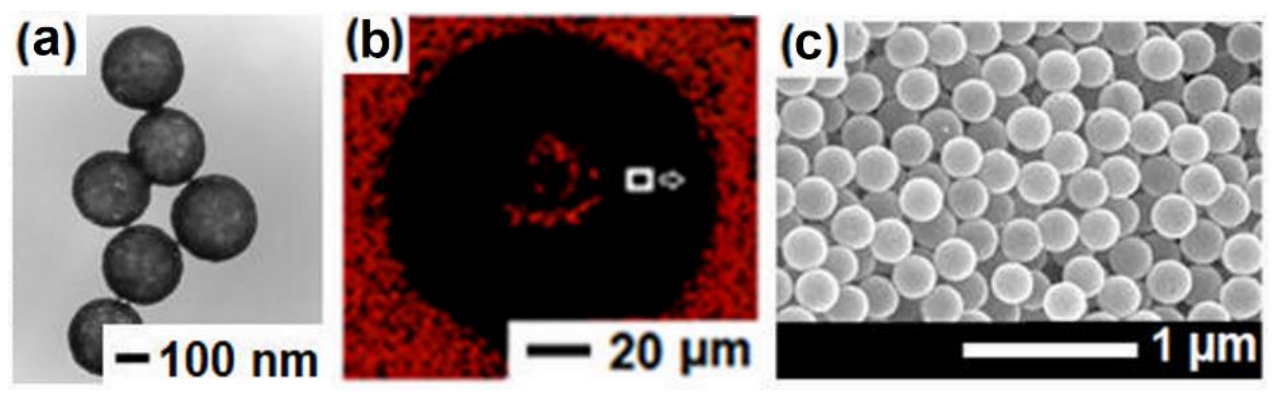

Figure 4.7 (a) TEM image of $240 \mathrm{~nm}$ gold-nanoshells consist of $200 \mathrm{~nm}$ silica core coated by $20 \mathrm{~nm}$ gold nanospheres (b) optical image of a nanogap-rich structure fabricated by 240 nm gold- nanoshells (c) SEM image of the area of nanogap-rich structure marked in (b) by a white box. ${ }^{23}$

\subsubsection{Fabrication of gold-nanostars-structure}

$100 \mathrm{~nm}$ gold nanostars purchased from 'Creative Diagnostics' was used in this experiment to fabricate gold-nanostars-structure. Gold nanostars solution was used as received without any further modification. Nanogap-rich structures fabricated from gold nanostars are referred in this research as gold-nanostar-structures.
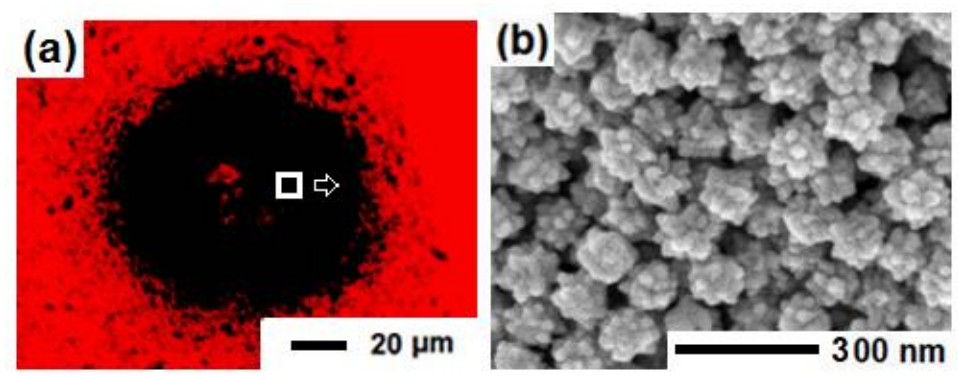

Figure 4.8 (a) Optical image of a nanogap rich structure fabricated from $100 \mathrm{~nm}$ gold nanostars (b) SEM image of the corresponding area of nanogap-rich structure marked in (a) by a white box. 
The optical images of a gold-nanostar-structure with $100 \pm 1 \mu \mathrm{m}$ diameters is shown in Figure 4.8 (a). Figure 4.8 (b) depicts the scanning electron microscope (SEM) images of the corresponding area of gold-nanostars-structure which is marked by a white box in Figure 4.8 (a).

\subsection{Fabrication parameters affecting the size of nanogap-rich structures}

There are several parameters which could affect the size of fabricated nanogap-rich structures during the fabrication process. These are as follows:

\subsubsection{Effect of laser intensity}

The size of nanogap-rich structure depends on the intensity of heating laser and can be controlled by changing the intensity of laser on the substrate. Figure 4.9 depicts the size of nanogap-rich structures as a function of laser intensity.

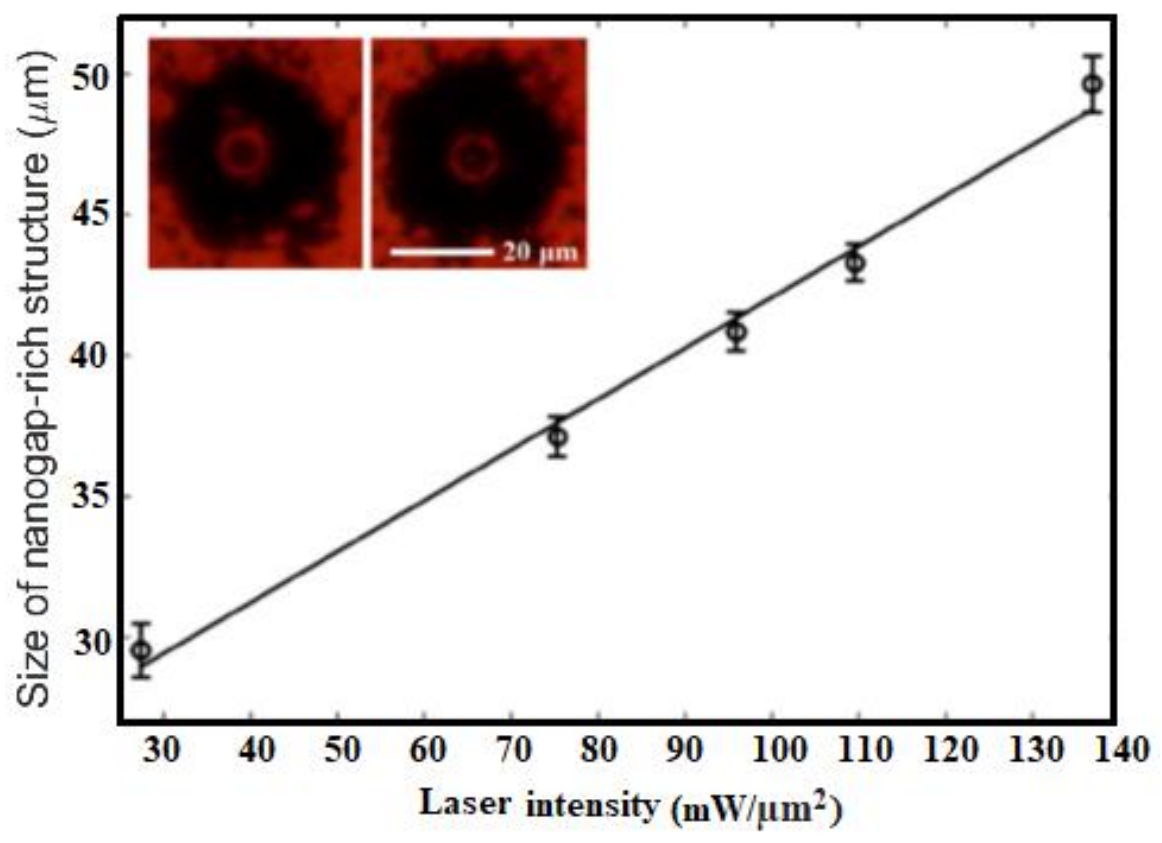

Figure 4.9 Size of nanogap-rich structure as a function of laser intensity. The experimental data with error bars are linearly fitted by a solid line. ${ }^{22}$ 
The solid curve in Figure 4.9 shows a linear fit of experimental data (shown as circles) with the function, $y=0.18 \mathrm{x}+24$, where $\mathrm{y}$ is the size of nanogap-rich structure in $\mu \mathrm{m}$ and $\mathrm{x}$ is the laser intensity in $\mathrm{mW} / \mathrm{\mu m}^{2}$ which is used during the fabrication of nanogap-rich structure. Inset in Figure 4.9 shows the optical images of two nanogap-rich structures fabricated from $200 \mathrm{~nm}$ gold nanospheres on the same gold-coated glass substrate under the same laser intensity. The laser exposure time was 45 seconds for all the experimental data. According to Figure 4.9, the size of nanogap-rich structure increases with the increasing of laser intensity.

\subsubsection{Effect of laser exposure time}

Figure 4.10 shows the change of the size of nanogap-rich structure as a function of laser exposure time.

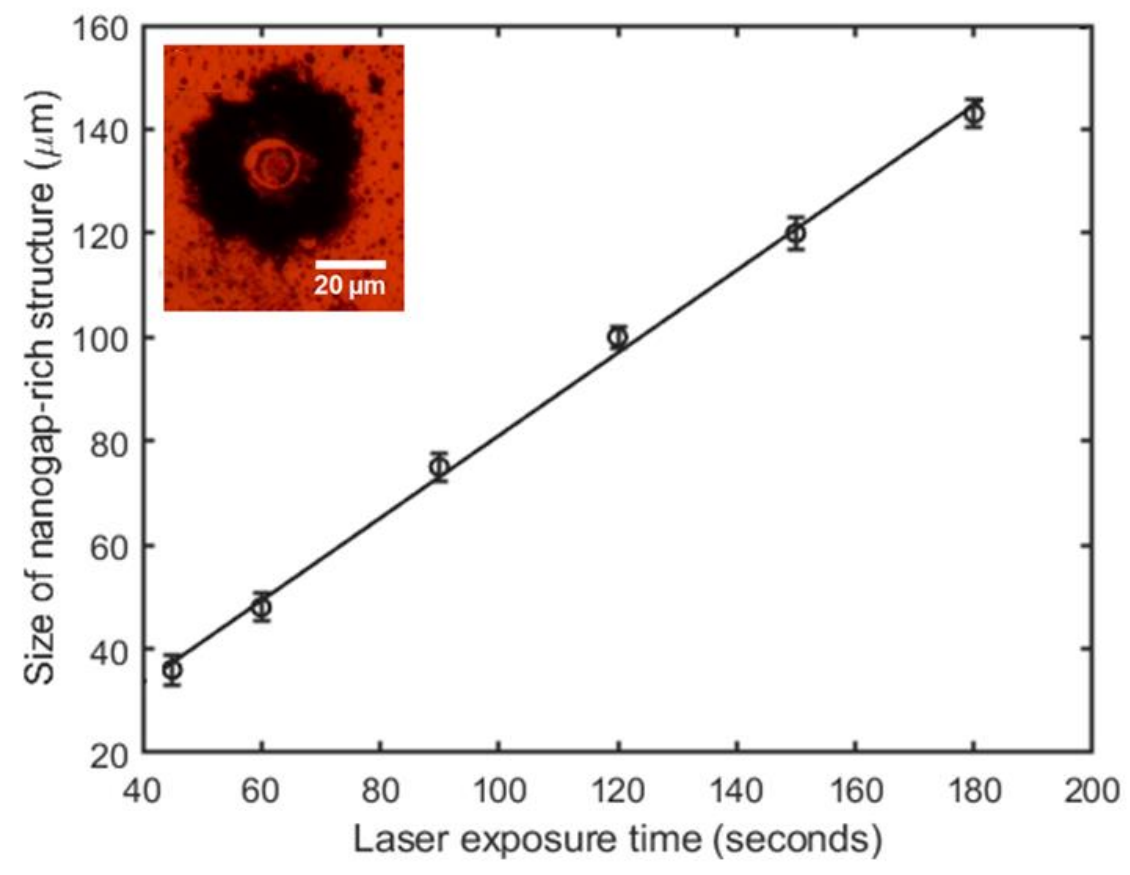

Figure 4.10 Size of nanogap-rich structure as a function of laser exposure time. The experimental data with error bars are linearly fitted by a solid line. 
The size of nanogap-rich structures inceases linearly with the exposure time. A linear fit of experimental data is illustrated by the solid line in Figure 4.10 with a relationship, $y=0.79 \mathrm{x}$ +1.6 , where $\mathrm{y}$ is the size of the nanogap-rich structure in $\mu \mathrm{m}$ and $\mathrm{x}$ is the laser exposure times in seconds. The inset shows one of the nanogap-rich structures fabricated from gold nanospheres. The laser intensity was $35 \mathrm{~mW} / \mu \mathrm{m}^{2}$ for all the experimental data.

\subsection{Stability of nanogap-rich structures}

We have tested the stability of nanogap-rich structures through the sonication of nanogaprich structure in DI water for 10 minutes. Figure 4.11 (a) and Figure 4.11 (b) illustrate nanogap-rich structures before and after sonication respectively. From the sonication results as depicted in Figure 4.11 we have seen that, the nanogap-rich structure is very stable even after 10 minutes sonication. Therefore, nanogap-rich structures fabricated by OGMB in our research are stable and can withstand under the harsh enviroment.

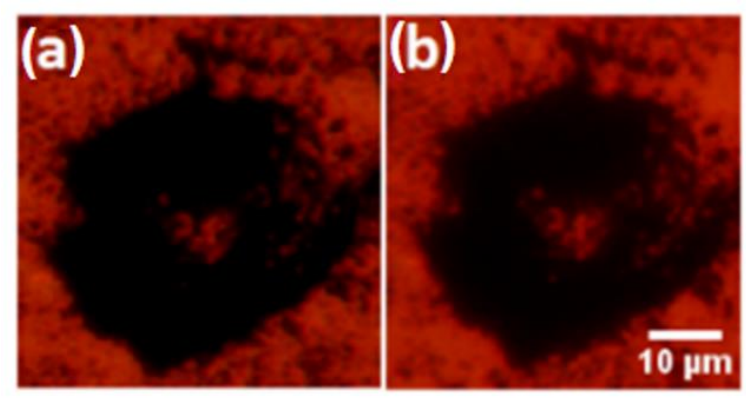

Figure 4.11 Nanogap-rich structure (a) before sonication (b) after 10 minutes sonication. ${ }^{23}$

\subsection{Effect of gold-coating on substrate for nanogap-rich structures fabrication}

It is mentioned in section 4.1 of this chapter that, gold coating on glass coverslip is not a pre-requirement for generatiing an OGMB. It is also reported in one of our publications ${ }^{22}$ that, OGMB can be also generated on a glass coverslip without gold coating. In order to 
prove this statement, we have generated an OGMB on a glass coverslip without gold coating. Figure $4.12(a-b)$ illustrate two gold-nanosphere-structures which were fabricated on a glass coverslip with $10 \mathrm{~nm}$ gold-coated film and without gold film respectively at a laser intensity of $34 \mathrm{~mW} / \mu \mathrm{m}^{2}$ and $171 \mathrm{~mW} / \mu \mathrm{m}^{2}$, respectively. Laser exposure time used during the fabrication of these two nanogap-rich structures was 2 minutes.
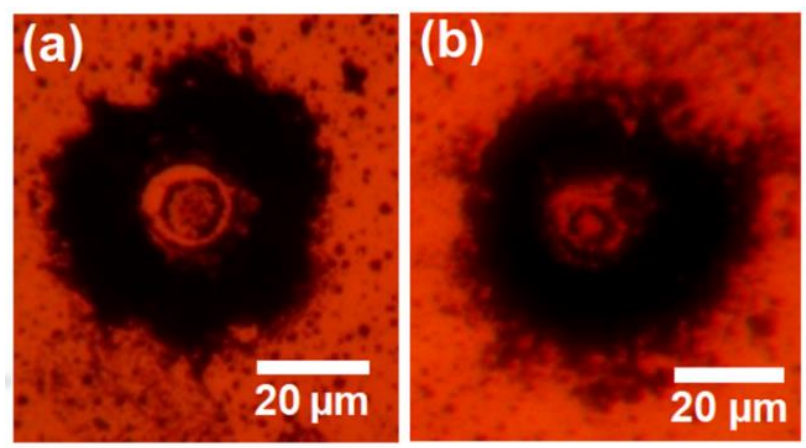

Figure 4.12 Nanogap-rich structure fabricated from $200 \mathrm{~nm}$ gold-nanosphere on a cover glass (a) coated with $10 \mathrm{~nm}$ gold film (b) without gold coating. ${ }^{23}$

These experimental results demonstrate that, the laser intensity required for the fabrication of nanogap-rich structures on a cover glass with $10 \mathrm{~nm}$ gold-coated film is five times lower than that on a cover glass without gold coating. Therefore, gold coating can reduce the laser intensity which is needed to generate an OGMB. This reduced laser intensity requirement for the fabrication of nanogap-rich structure on a glass coverslip with gold coating could be the result of enhanced laser heating effect due to gold flim. In order to verify this statement, we have estimated the temperature distribution of a $200 \mathrm{~nm}$ gold nanosphere with and without gold coating through COMSOL Multiphysics simulation. Figure 4.13 (ab) shows the simulated profile of the temperature distribution of a 200-nm gold nanosphere on a cover glass coated with 10 -nm thick gold film and without gold film respectively. According to these simulation results as shown in Figure 4.13, under the same laser 
intensity, the highest temperature of a $200 \mathrm{~nm}$ gold nanosphere on a cover glass substrate without any gold film is $345 \mathrm{~K}$ which is more than $900 \mathrm{~K}$ in case of $10 \mathrm{~nm}$ gold-coated film. Therefore, the temperature of a $200 \mathrm{~nm}$ gold nanosphere on a cover glass substrate with $10 \mathrm{~nm}$ gold-coated film is approximately three times higher than that on a glass coverslip without gold coating.
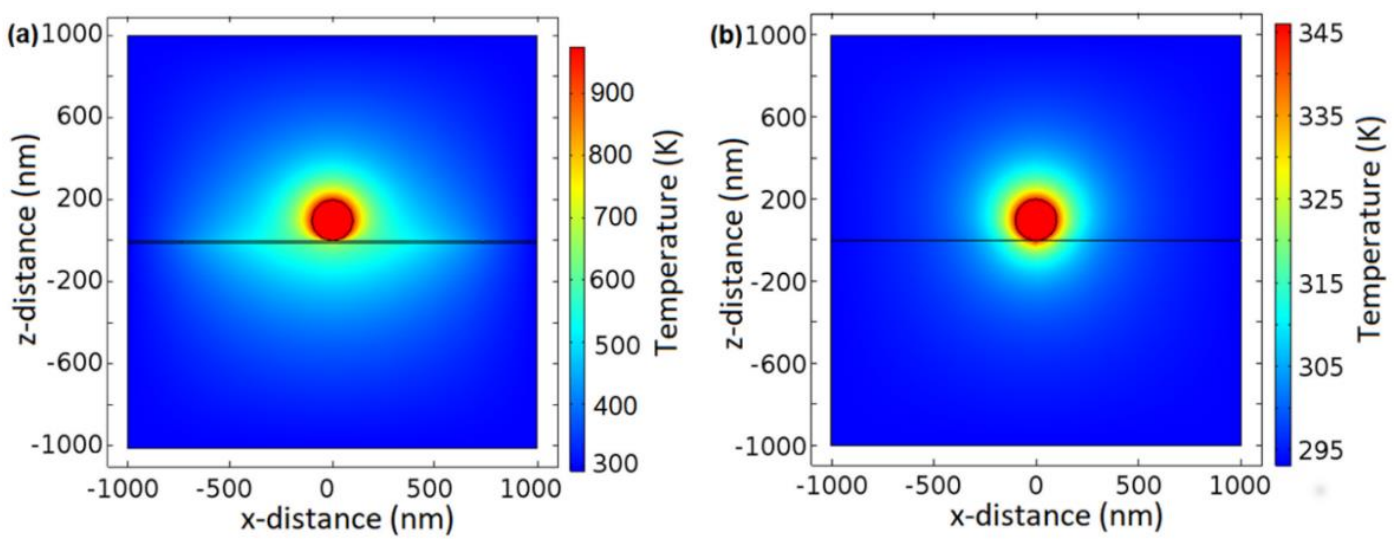

Figure 4.13 Simulated profile of temperature distribution of $200 \mathrm{~nm}$ gold-nanosphere on a glass coverslip substrate (a) with gold-coating (b) without gold-coating. Simulation was performed by COMSOL Multiphysics. ${ }^{23}$

Due to this higher temperature of $200 \mathrm{~nm}$ gold nanosphere on a gold-coated glass substrate, we are able to generate an OGMB at a comparetively lower laser intensity compared to that on a glass coverslip without gold coating.

In this work, nanogap-rich structures can be rapidly fabricated through an OGMB on any kind of subtrate under ambient conditions. No special substrate or special environment such vacuum environment is needed to fabricate the nanogap-rich structure. Therefore, the fabrication process of nanogap-rich structure applied in this work is a rapid and affordable fabrication method. 


\section{CHAPTER 5}

\section{OGMB BASED NANOGAP-RICH STRUCTURES FOR CHEMICAL SENSING}

In chapter 4 we have explained in detail the fabrication process of nanogap-rich structures through an OGMB. The fabricated nanogap-rich structures can be used for chemical and biosensing application. In this chapter, we will explain the active chemical sensing application of OGMB-based nanogap-rich structures through the SERS detection of two chemicals; Rhodamine 6G (R6G) and Malachite Green (MG).

\subsection{Develop an ultrasensitive chemical and biosensing platform}

After the fabrication of nanogap-rich structure, we have developed an ultrasensitive chemical and biosensing platform through the attachment of analytes on the fabricated nanogap-rich structure.

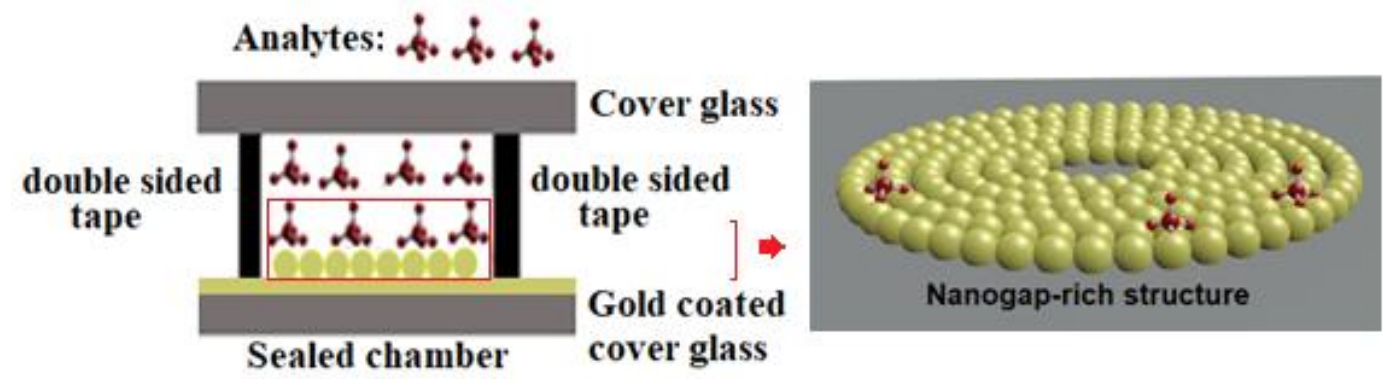

Figure 5.1 Schematic of ultra-sensitive chemical and biosensing platform.

Figure 5.1 schematically shows the sensing platform. Chemical or biological samples were contained in a sealed chamber, which consists of a cover-glass at the top surface and a gold-coated cover glass along with the fabricated nanogap-rich structure as the bottom surface. A double-sided tape is sandwiched between the two substrates as a spacer. 


\subsection{Nanogap-rich structures for active and ultrasensitive chemical sensing}

In this section, we are going to discuss the application of two types of nanogap-rich structures; gold-nanosphere-structure and gold-nanoshell-structure fabricated from $200 \mathrm{~nm}$ gold nanospheres and $240 \mathrm{~nm}$ gold nanoshells (silica core) respectively, for active and ultrasensitive chemical sensing. The OGMB-based active chemical sensing has been demonstrated through the SERS detection of two chemicals; Rhodamine 6G (R6G) and Malachite Green (MG).

At first, we have tested gold-nanosphere structure both for passive and active chemical sensing application through the SERS detection of R6G. In the following section, we will describe the performance of gold-nanosphere-structure both for the application of passive and active chemical sensing.

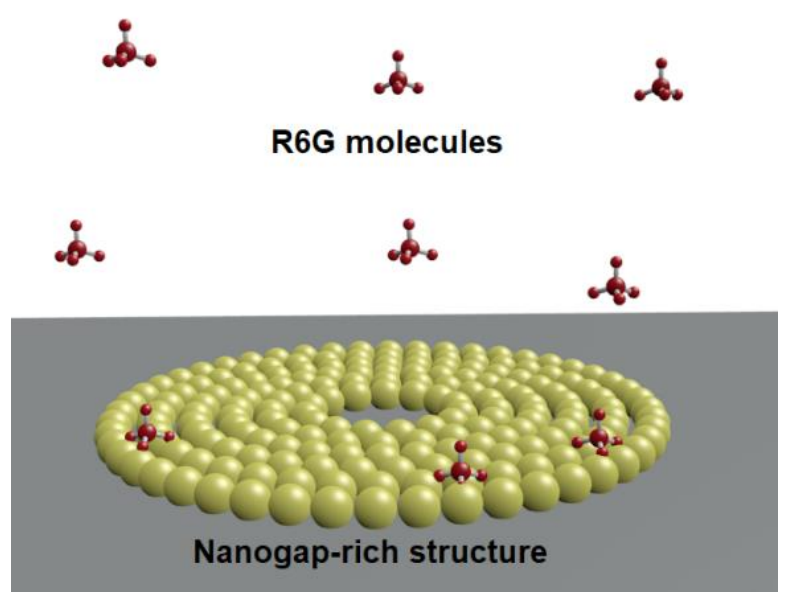

Figure 5.2 Schematic of free diffusion of R6G molecules towards nanogap-rich structure: an example of passive sensing. ${ }^{23}$

\subsubsection{Gold-nanosphere-structure for passive chemical sensing}

The performance of gold-nanosphere-structure fabricated from $200 \mathrm{~nm}$ gold-nanospheres was tested under passive sensing condition through the SERS measurement of R6G 
molecules at different concentrations. During passive sensing, R6G molecules were freely diffused towards the gold-nanosphere-structure as schematically shown in Figure 5.2. Then the SERS spectra of freely diffused R6G on gold-nanosphere-structure were recorded by a Raman spectrometer (Renishaw inVia Reflex Micro-Raman) as shown in Figure 5.3. The Raman spectrometer is equipped with a near infrared diode laser source of wavelength $\lambda=785 \mathrm{~nm}$. The maximum power of laser source in the Raman spectrometer is $300 \mathrm{~mW}$. The laser (785 nm wavelength) used during the SERS measurement of analytes in this experiment is different from the heating laser $(1064 \mathrm{~nm})$ used for the fabrication of nanogap-rich-structures. However, it is worth noting that heating laser and Raman exciting laser could be the same in order to simplify the experimental setup.

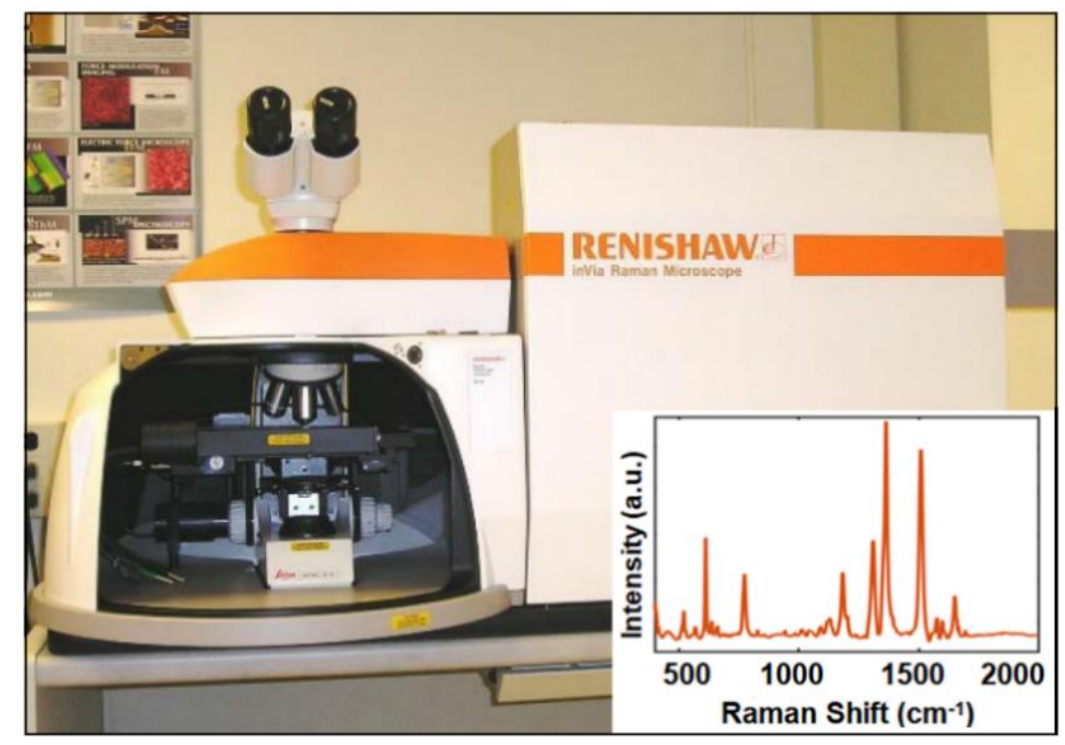

Figure 5.3 Renishaw inVia Reflex Micro-Raman used for the SERS measurement of R6G molecules. Inset: SERS spectra of R6G recoded by this Raman spectrometer.

During the SERS measurement of analytes, $30 \mathrm{mw}$ laser power (10\% of maximum power of diode laser source) was used. Acquisition time of Raman measurement was 10 seconds. 

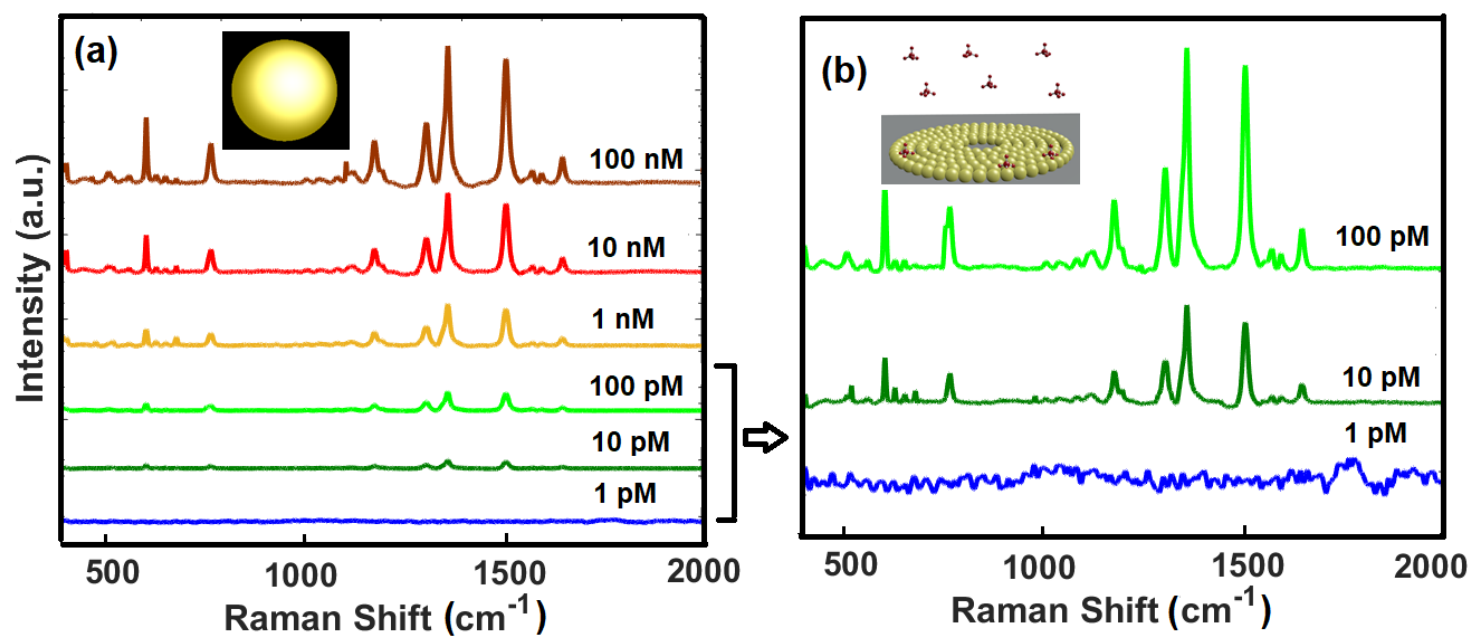

Figure 5.4 (a) SERS spectra of R6G at different concentrations (from $100 \mathrm{nM}$ to $1 \mathrm{pM}$ ) collected by gold-nanosphere-structure through passive sensing method. Inset: schematic of a gold nanoshere used for the fabrication of gold-nanosphere-structure (b) magnified image of SERS spectra of R6G at lower concentrations (from $100 \mathrm{pM}$ to $1 \mathrm{pM}$ ) marked in (a) by an arrow. Inset: schematic of passive sensing methods. ${ }^{23}$

The Raman exciting laser were focused on R6G molecules on the gold-nanospherestructure through a $50 \times$ microscope objective lens with numerical aperture, $\mathrm{NA}=0.75$. Raman exciting laser were carefully focused on the analytes on the nanogap-rich structure in order to get maximum Raman signal from the sample.

The SERS spectra of R6G at different concentration under passive sensing condition is shown in Figure 5.4. Though the SERS measurement of R6G, the following Raman peaks of R6G were clearly observed: C-C stretching at $1364 \mathrm{~cm}^{-1}, 1510 \mathrm{~cm}^{-1}, 1651 \mathrm{~cm}^{-1}, \mathrm{~N}-\mathrm{H}$ in-plane bending at $1310 \mathrm{~cm}^{-1}, 1575 \mathrm{~cm}^{-1}, \mathrm{C}-\mathrm{H}$ in-plane bending at $1130 \mathrm{~cm}^{-1}, 1183 \mathrm{~cm}^{-1}$, $\mathrm{C}-\mathrm{H}$ out-of-plane bending at $775 \mathrm{~cm}^{-1}, \mathrm{C}-\mathrm{C}-\mathrm{C}$ ring in-plane vibration at $613 \mathrm{~cm}^{-1}$. These Raman peaks detected through our experiment are closely matches with the characteristic Raman peaks of R6G reported in the literature..$^{98,99}$ 
SERS spectra of lower concentrations of R6G in Figure 5.4 (a) are also illustrated in a magnified image in Figure 5.4 (b). According to Figure 5.4 (a-b), SERS intensity of R6G decreases with reducing the concentrations of R6G. Through these SERS measurements, R6G is detectable up to $10 \mathrm{pM}$ concentration by gold-nanosphere-structure under passive sensing condition as shown in Figure 5.4 (b).

\subsubsection{Gold-nanosphere-structure for active chemical sensing}

Active sensing of analytes means actively guide as well as concentrate analytes towards the surface of a sensor. For example, by applying active sensing method R6G molecules is actively accumulated on a gold film as shown in Figure 5.5.

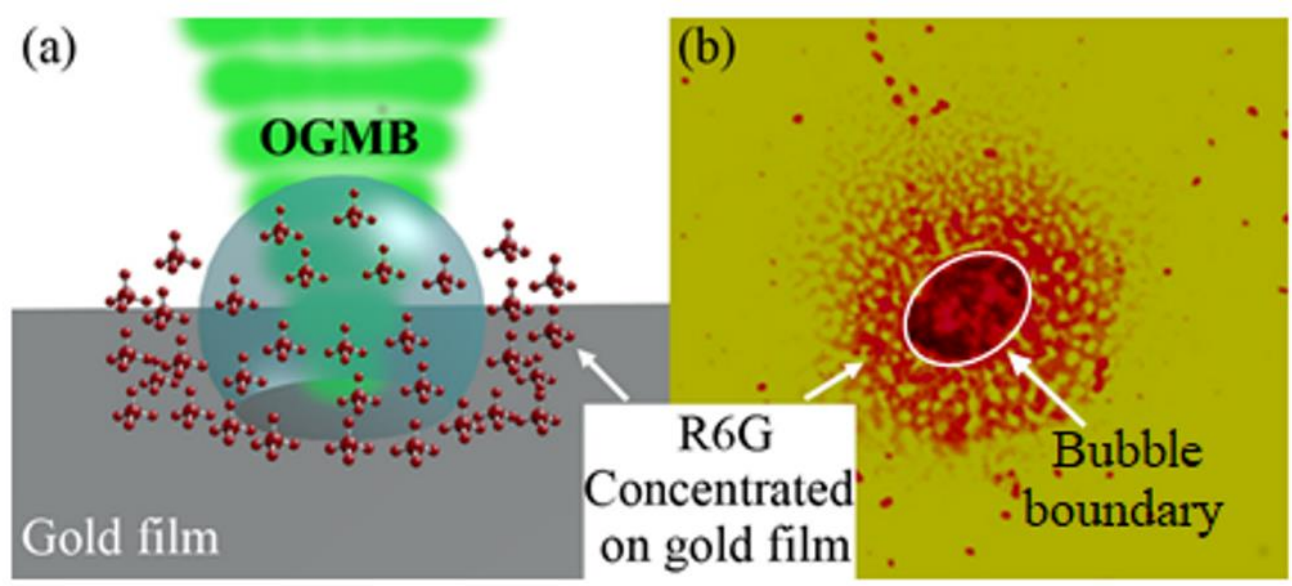

Figure 5.5 (a) Schematic of R6G accumulation on a gold film through an OGMB. (b) optical image of R6G concentrated on gold film through the generation of an OGMB from R6G solution. ${ }^{23}$

In order to apply this active sensing method, an OGMB is generated inside a droplet of R6G solution on a gold film as schematically depicted in Figure 5.5 (a). Due to the Marangoni convective flow induced by OGMB, R6G molecules are guided around OGMB and then concentrated on the gold film as schematically shown in Figure 5.5 (a). 
A laser intensity of $10 \mathrm{~mW} / \mu \mathrm{m}^{2}$ is used for the active sensing of analytes. The high temperate only affect the area inside the OGMB. The analytes outside of the OGMB is isolated from the laser illuminating area once a microbubble is generated as schematically shown in Figure 5.5 (a). Since the analytes cannot go into the microbubble; therefore, the analytes will not reach the "hottest area" (laser illuminating area) once a microbubble is generated. However, some analytes may go into the "hottest area" at the beginning of the microbubble generation, which may be affected by the high temperature. Figure 5.5 (b) shows the potential damage of R6G inside and outside of a microbubble on a gold-coated glass substrate. It is obvious that, only the analytes inside the microbubble are damaged as marked by the black region in Figure 5.5 (b). The analytes outside of the microbubble are "safe". The Raman measurements in this work were conducted on the undamaged areas of the concentrated R6G, which are the outside areas of the microbubble boundary as shown in Figure $5.5(b)$.

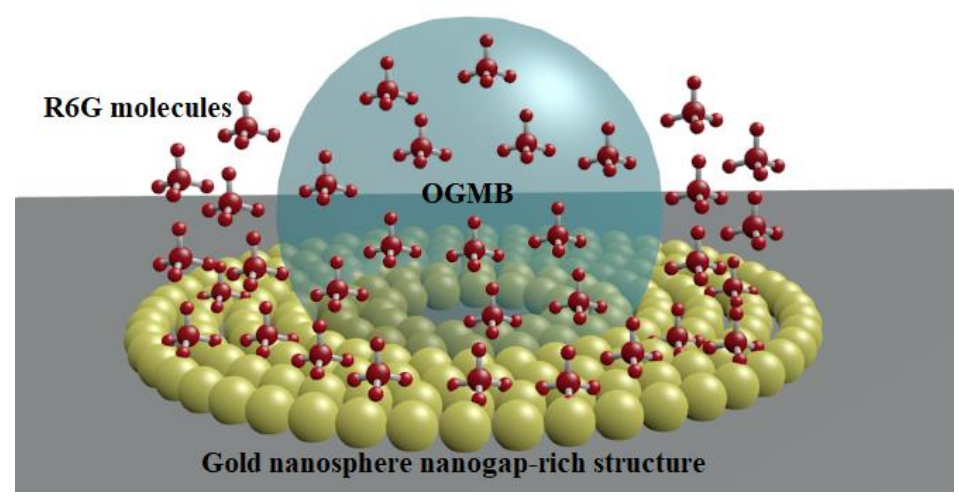

Figure 5.6 Schematic of actively guide and concentrate R6G molecules towards nanogaprich structure through an OGMB: an example of active sensing. ${ }^{23}$

In order to test the performance of gold-nanosphere-structure under the active sensing method, an OGMB is generated on gold-nanosphere-structure through the laser heating of 
gold-coated cover glass containg R6G solution. Due to the strong Marangoni convective flow associated with OGMB, R6G molecules are guided and forced to accumulate on the gold-nanosphere-structure as schematically shown in Figure 5.6. Under active sensing condition, SERS spectra of R6G at different concentrations are recorded after R6G molecules are guided and concentrated on the gold-nanosphere-structure through an OGMB generated from R6G solution.
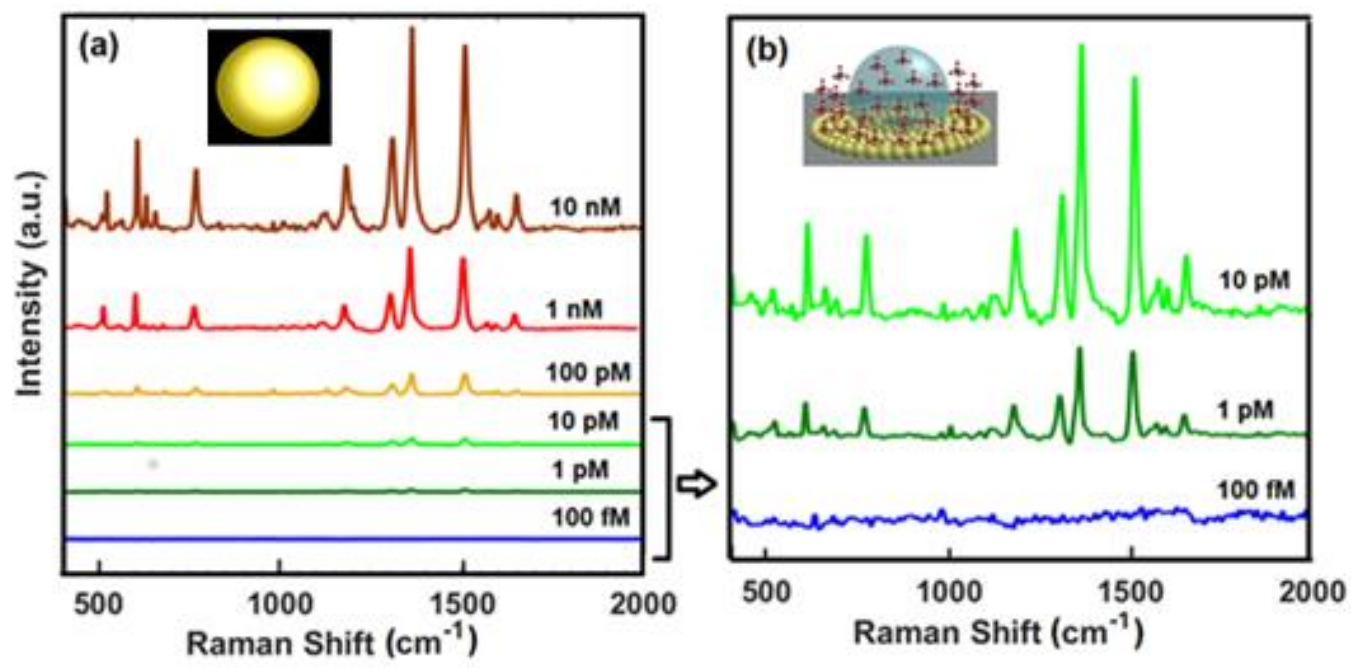

Figure 5.7 (a) SERS spectra of R6G at different concentrations (from $10 \mathrm{nM}$ to $100 \mathrm{fM}$ ) collected by gold-nanosphere-structure under active sensing method. Inset: schematic of a gold nanoshere used for the fabrication of gold-nanosphere-structure (b) magnified image of SERS spectra of R6G at lower concentrations (from $10 \mathrm{pM}$ to $100 \mathrm{fM}$ ) marked in (a) by an arrow. Inset: schematic of active sensing methods. ${ }^{23}$

Similar experimental condition of passive sensing i.e. $785 \mathrm{~nm}$ laser wavelength, 10 seconds acquisition time and $30 \mathrm{~mW}$ laser power are used to record the SERS spectra of R6G under active sensing condition. SERS spectra of R6G at different concentrations using active sensing method is depicted in Figure 5.7(a). SERS spectra of R6G at lower concentrations is also shown in Figure 5.7 (b). The detection limit of gold-nanosphere-structure through 
the active sensing method is $1 \mathrm{pM}$ as shown in Figure 5.7 which is not detectable by the same gold-nanosphere-structure under the passive sensing condition.

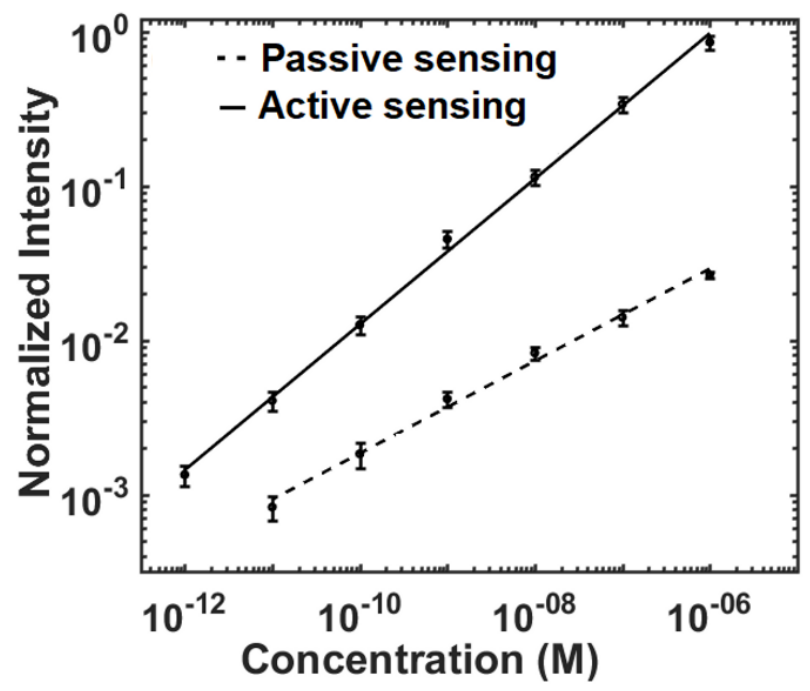

Figure 5.8 Normalized SERS intensity of R6G at $1364 \mathrm{~cm}^{-1}$ as a function of R6G concentration through active sensing (solid line) and passive sensing (dashed line) methods with error bars indicating experimental standard deviations. ${ }^{23}$

Figure 5.8 depicts the normalized SERS intensity of $\mathrm{R} 6 \mathrm{G}$ at $1364 \mathrm{~cm}^{-1}$ due to passive sensing (dashed line) and active sensing (solid line) as a function of R6G concentration. Experimental error bars of SERS intensity of R6G at $1364 \mathrm{~cm}^{-1}$ both for the active and passive sensing are calculated from four sets of SERS spectra of R6G recorded at four different positions of gold-nanosphere-structure. Experimental data are fitted by the functions as stated below:

Active sensing: $\log _{10}(y)=0.47 \log _{10}(x)+2.82 \quad$ with $\mathrm{R}^{2}=0.998$

Passive sensing: $\log _{10}(y)=0.30 \log _{10}(x)+0.26$ with $\mathrm{R}^{2}=0.994$ 
Where, $x$ is the concentration of R6G, $y$ represents normalized SERS intensity of different concentration of R6G at $1364 \mathrm{~cm}^{-1}$. According to the data depicted in Figure 5.8, a higher sensitivity is observed in case of gold-nanosphere-structure due to active sensing compared to the sensitivity achieved due to passive sensing.

\subsubsection{SERS enhancement factor of gold-nanosphere-structure due to passive sensing}

The average SERS enhancement factor (EF) of gold-nanosphere-structure was calculated through the comparison of SERS signals collected from $0.1 \mu \mathrm{M}$ R6G on gold-nanospherestructure and Raman signals recorded for $10 \mathrm{mM} \mathrm{R6G}$ in the absence of gold-nanospherestructure by using the formula as stated below: ${ }^{100}$

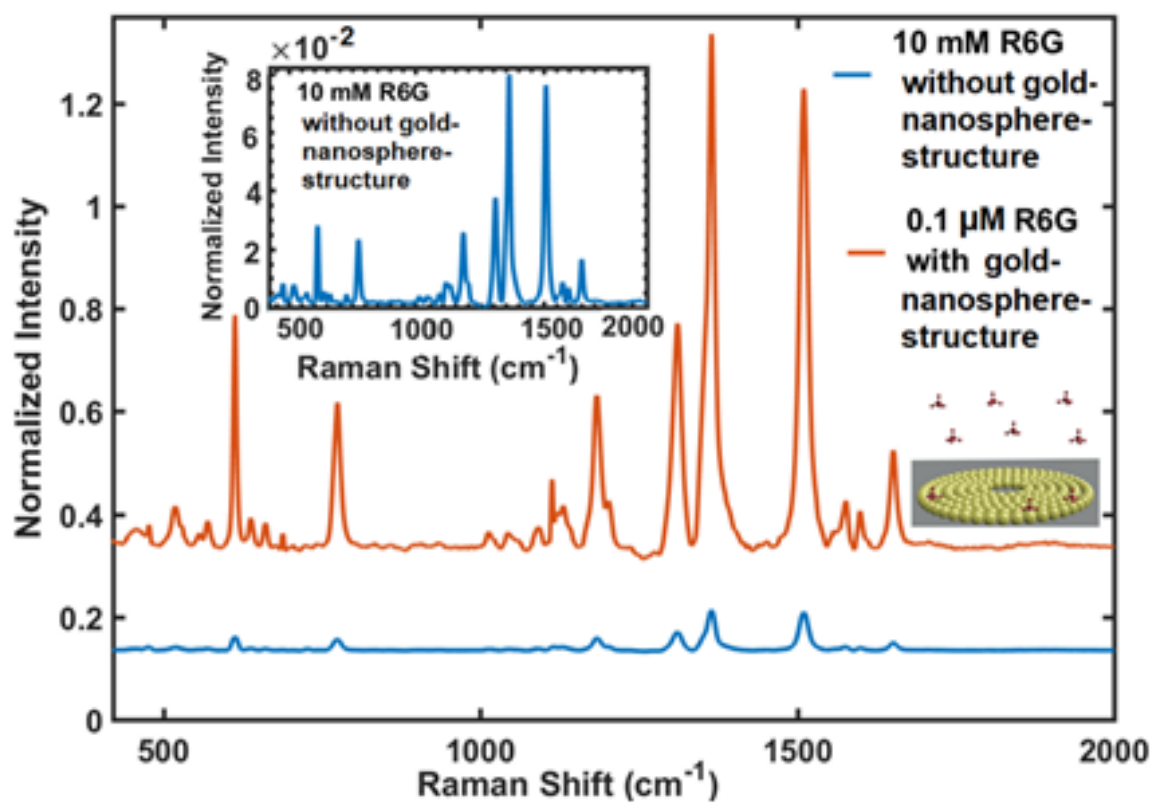

Figure 5.9 Normalized Raman intensity of R6G molecules at $1364 \mathrm{~cm}^{-1}$ without goldnanosphere-structure (blue curve) and with gold-nanosphere-structure (red curve). Inset (top) illustrates magnified Raman spectrum of $10 \mathrm{mM}$ R6G without gold-nanospherestructure. Inset (bottom) demonstrates passive sensing is used to obatain these data. ${ }^{23}$

$$
E F=\frac{I_{S E R S}}{I_{R S}} \times \frac{C_{R S}}{C_{S E R S}}
$$


Where, $I_{\text {SERS }}=$ SERS intensities of R6G at $1364 \mathrm{~cm}^{-1}$ on gold-nanosphere-structure. $I_{R S}=$ Raman intensities of R6G at $1364 \mathrm{~cm}^{-1}$ in absence of gold-nanosphere-structure. $C_{R S}=10 \times 10^{-3} \mathrm{M}$ concentration of R6G for normal Raman measurement. $C_{\text {SERS }}=1 \times 10^{-7} \mathrm{M}$ concentration of R6G for SERS measurement.

Figure 5.9 illustrates normalized SERS intensity of $0.1 \mu \mathrm{M} \mathrm{R} 6 \mathrm{G}$ at $1364 \mathrm{~cm}^{-1}$ with goldnanosphere-structure (red curve) and the corresponding Raman intensity of $10 \mathrm{mM} \mathrm{R6G}$ at $1364 \mathrm{~cm}^{-1}$ without gold-nanosphere-structure (blue curve). Passive sensing method was applied to record these data. Average SERS enhancement factor (EF) of gold-nanospherestructure is $1.2 \times 10^{6}$ which was calculated from four sets of experimental data according to the above formula. This SERS EF of gold-nanosphere-structure is comparable to the SERS EF for many conventional SERS substrates reported in the literature. ${ }^{67,100-102}$

\subsubsection{Enhancement factor of gold-nanosphere-structure due to active sensing}

Active sensing can provide an additional enhancement factor of Raman signal besides the SERS enhancement factor of gold-nanosphere-structure. Analytes concentration due to active sensing is responsible for this additional enhancement factor. It should be mentioned here that, enhancement because of analytes concentration is different from SERS enhancement. SERS enhancement is a result of enhanced electric field presents between the nano-gaps of gold-nanosphere-structure.

On the other hand, enhancement factor due to active sensing is the result of OGMB-assisted strong convective flow-based analytes concentration. This additional enhancement factor which is achieved because of analytes concentration through active sensing is calculated 
by the ratio of SERS peak of R6G at $1364 \mathrm{~cm}^{-1}$ for active sensing (solid line of Figure 5.8) to that for passive sensing (dashed line of Figure 5.8) for the same concentration of R6G. The enhancement factor because of analytes concentration through active sensing of analytes as a function of R6G concentration is depicted in Figure 5.10.

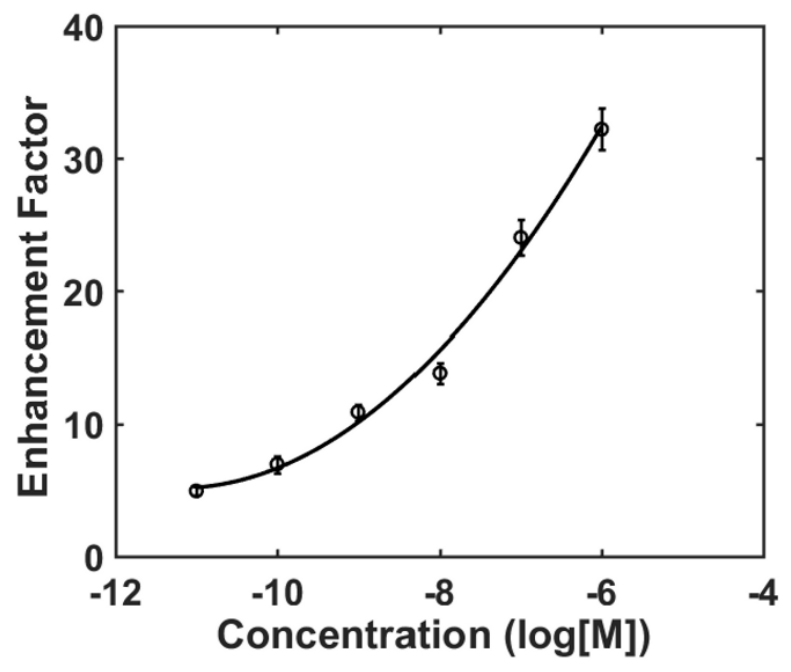

Figure 5.10 Enhancement factor of gold-nanosphere-structure as a function of R6G concentrations due to active sensing of analyte, R6G. ${ }^{23}$

Experimental data is fitted by the function as stated below:

$$
y=0.99 x^{2}+22.33 x+130.59 \quad \text { with } \mathrm{R}^{2}=0.991
$$

Where, $x$ represents log of R6G concentration and $y$ represents enhancement factor happens because of analytes concentration.

Besides the SERS enhancement factor, $1.2 \times 10^{6}$ of gold-nanosphere-structure, the additional enhancement factor of around $5 \pm 0.3$ and $32 \pm 1.5$ are achieved for $10 \mathrm{pM}$ and 1 $\mu \mathrm{M}$ concentration of $\mathrm{R} 6 \mathrm{G}$, respectively due to the analytes concentration through active sensing. According to these experimental data, higher concentration of analytes provides a 
higher enhancement factor since the molecules have higher chance to be captured and then concentrated on the surface of gold-nanosphere-structure due to strong convective flow induced by the OGMB.

\subsubsection{Gold-nanoshell-structure for passive chemical sensing}

From the above discussion we have seen that, detection limit of gold-nanosphere-structure is up to $1 \mathrm{pM}$ R6G by applying the active sensing method. However, the detection limit can be further improved through another nanogap-rich-structure fabricated from gold nanoshell known as gold-nanoshell-structure.

Similar like gold-nanosphere-structure, gold-nanoshell-structure is also used both for passive and active chemical sensing application. SERS spectra of R6G molecules at different concentrations collected by gold-nanoshell-structure using the passive sensing method is depicted in Figure 5.11 (a).
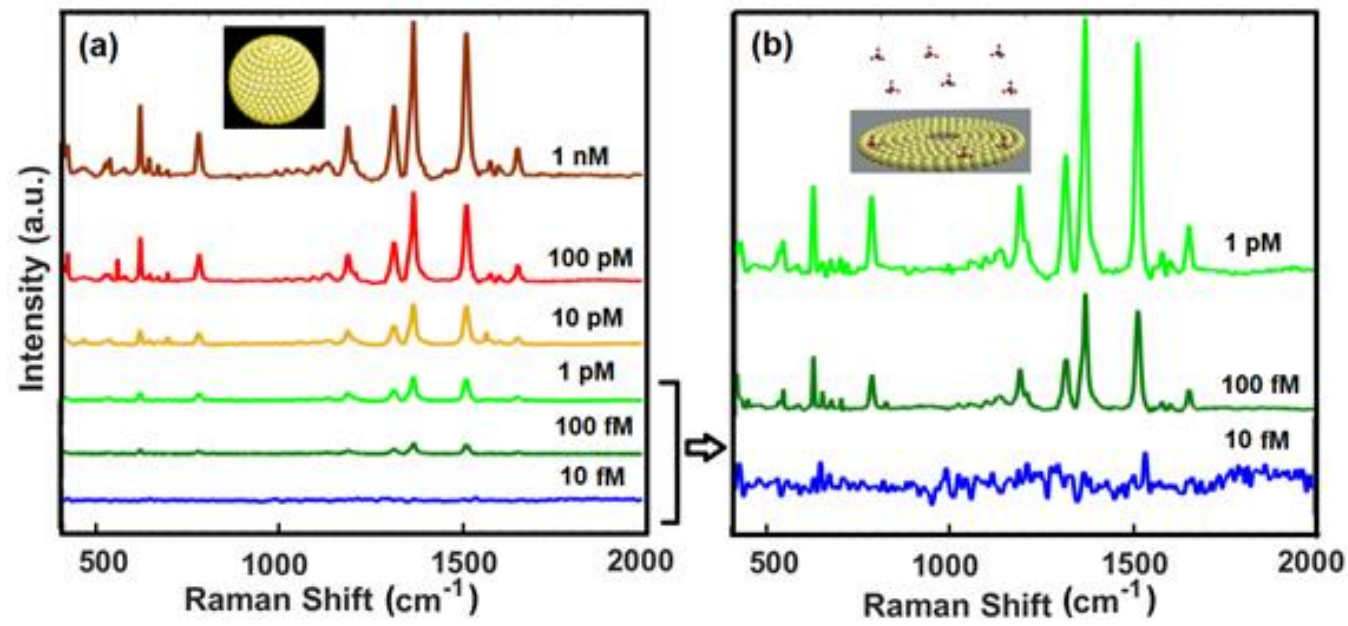

Figure 5.11 (a) SERS spectra of different concentrations (from $1 \mathrm{nM}$ to $10 \mathrm{fM}$ ) of R6G collected by gold-nanoshell-structure through passive sensing. Inset: schematic of a gold nanoshell used for the fabrication of gold-nanoshell-structure (b) magnified image of SERS spectra of R6G corresponding to lower concentrations (from $1 \mathrm{pM}$ to $10 \mathrm{fM}$ ) marked in (a) by an arrow. Inset: schematic of passive sensing illustrates free diffusion of analytes towards nanogap-rich-structure. ${ }^{23}$ 
As described above, here passive sensing means R6G molecules are freely diffused towards the gold-nanoshell-structure as schematically shown in the inset of Figure 5.11 (b). The SERS spectra of R6G at lower concentrations are also depicted in Figure 5.11 (b).

The detection limit of gold-nanoshell-structure using the passive sensing method is 100 $\mathrm{fM}$, which is 10 times more sensitive than that from a gold-nanosphere-structure. The similar experimental condition (785 $\mathrm{nm}$ laser wavelength, $30 \mathrm{~mW}$ laser power, and 10 seconds laser exposure time) were used as in case of gold-nanosphere-structure.

\subsubsection{Gold-nanoshell-structure for active chemical sensing}

SERS spectra of R6G at different concentrations recorded by gold-nanoshell-structure using active sensing method is depicted in Figure 5.12 (a).
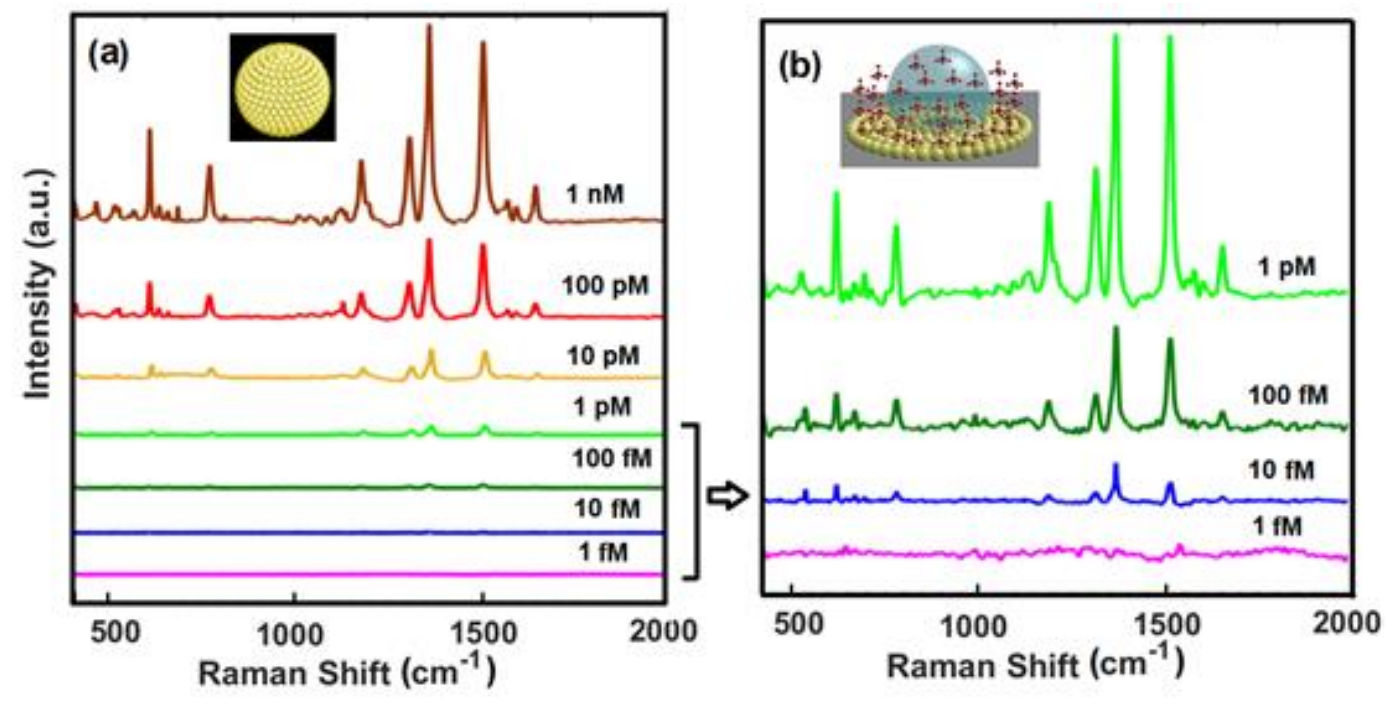

Figure 5.12 (a) SERS spectra of R6G at different concentration (from $1 \mathrm{nM}$ to $1 \mathrm{fM}$ ) collected by gold-nanoshell-structure through active sensing method. Inset: schematic of a gold nanoshell used for the fabrication of gold-nanoshell-structure (b) magnified image of SERS spectra of R6G at lower concentrations (from $1 \mathrm{pM}$ to $1 \mathrm{fM}$ ) as indicated in (a) by an arrow. Inset: schematic of active sensing of analytes demonstrates analytes are actively guided and concentrated towards nanogap-rich-structure. ${ }^{23}$ 
Here, active sensing means R6G molecules are actively guided and concentrated on goldnanoshell-structure through an OGMB as schematically shown in the inset of Figure 5.12 (b). The SERS spectra of R6G at lower concentrations (from $1 \mathrm{pM}$ to $1 \mathrm{fM}$ ) as marked in Figure 5.12 (a) by an arrow are depicted in Figure 5.12 (b). The detection limit of goldnanoshell-structure through the active sensing method is $10 \mathrm{fM}$ as shown in Figure 5.12 which is one order magnitude higher than the detection limit of same gold-nanoshellstructure under the passive sensing condition.

Normalized SERS intensity of R6G at $1364 \mathrm{~cm}^{-1}$, versus R6G concentration both for the passive sensing (dashed line) and active sensing (solid line) are illustrated in Figure 5.13. Experimental error bars of SERS intensity of R6G at $1364 \mathrm{~cm}^{-1}$ as shown in Figure 5.13, are calculated from four sets of SERS spectra of R6G recorded at four different positions of gold-nanoshell-structure.

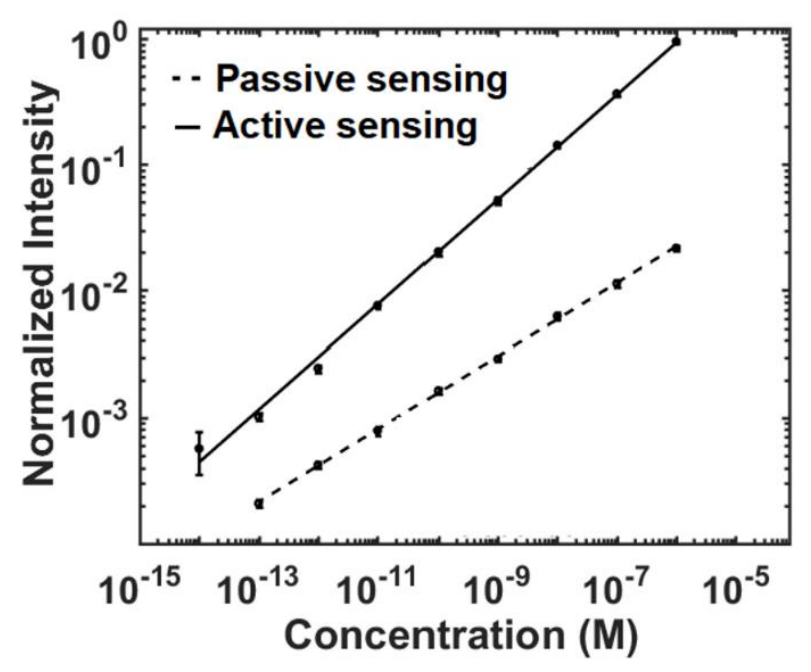

Figure 5.13 Normalized SERS intensity of R6G at $1364 \mathrm{~cm}^{-1}$ as a function of R6G concentration by active sensing (solid line) and passive sensing (dashed line). ${ }^{23}$

In Figure 5.13, experimental data are fitted by the functions as stated below:

Active sensing: $\log _{10}(y)=0.42 \log _{10}(x)+2.46 \quad$ with $\mathrm{R}^{2}=0.998$. 
passive sensing: $\log _{10}(y)=0.29 \log _{10}(x)+0.08 \quad$ with $\mathrm{R}^{2}=0.999$.

Here, $x$ is the concentration of R6G, and $y$ represents the normalized SERS intensity of R6G molecules at $1364 \mathrm{~cm}^{-1}$.

\subsubsection{SERS enhancement factor of gold-nanoshell-structure due to passive sensing}

Similar like gold-nanosphere-structure the average SERS enhancement factor of goldnanoshell-structure was investigated.

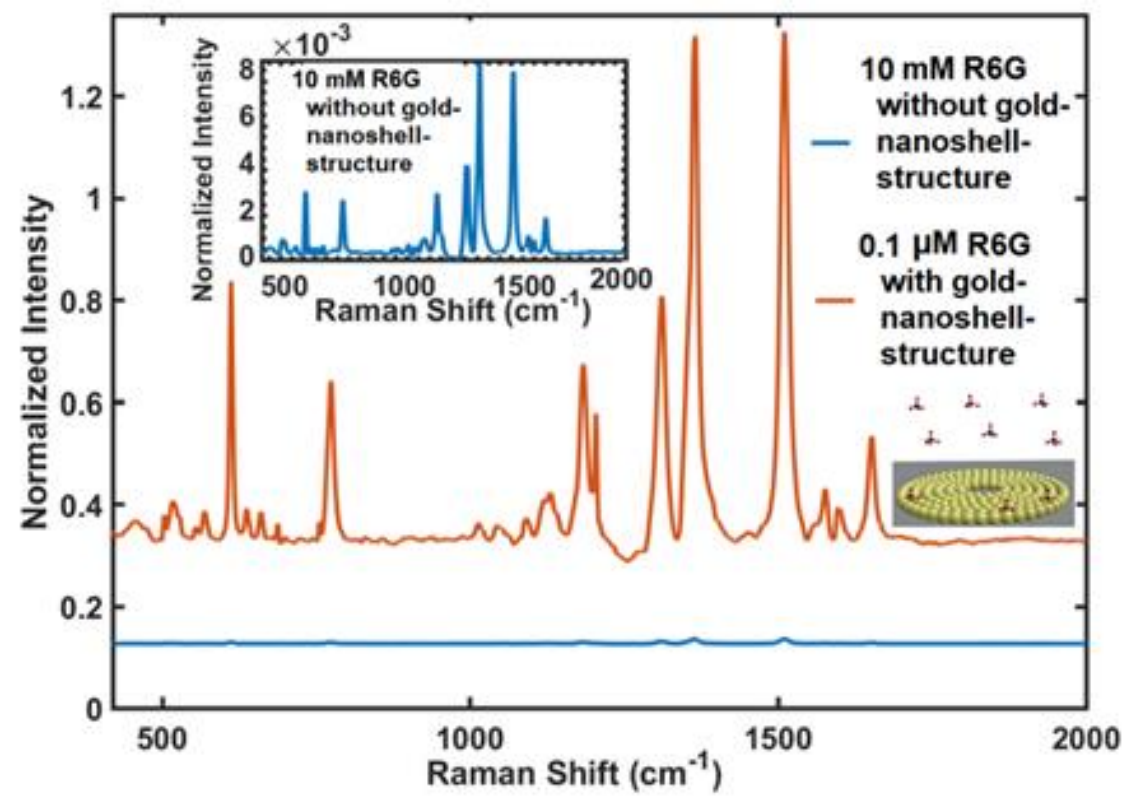

Figure 5.14 Normalized Raman intenisty of R6G at $1364 \mathrm{~cm}^{-1}$ without gold-nanoshellstructure (blue curve) and with gold-nanoshell-structure (red curve). Inset (top) depicts magnified Raman spectrum of $10 \mathrm{mM}$ R6G without gold-nanoshell-structure. Inset (bottom) illustrates schematic of passive sensing method is applied to record these data. ${ }^{23}$

Figure 5.14 depicts normalized SERS intensity of $0.1 \mu \mathrm{M}$ R6G at $1364 \mathrm{~cm}^{-1}$ collected by gold-nanoshell-structure (red curve) and the corresponding Raman intensity of $10 \mathrm{mM}$ R6G at $1364 \mathrm{~cm}^{-1}$ without gold-nanoshell-structure (blue curve). These data are recorded by passive sensing method. The average SERS enhancement factor of gold-nanoshell- 
structure was calculated through the comparison of SERS signals collected from $0.1 \mu \mathrm{M}$ R6G on gold-nanoshell-structure and the Raman signals recorded for $10 \mathrm{mM}$ R6G in absence of gold-nanoshell-structure according to the formula as stated in equation (5.3). Average SERS enhancement factor (EF) of gold-nanoshell-structure is $1.1 \times 10^{7}$ which was calculated from four sets of experimental data. This enhancement factor is one-order magnitude higher than the enhancement factor of gold-nanosphere-structure and also comparable to SERS enhancement factor for many conventional SERS substrates reported in the literature. ${ }^{67,100-102}$

\subsubsection{Enhancement factor of gold-nanoshell-structure due to active sensing}

Additional enhancement factor of gold-nanoshell-structure besides the SERS enhancement is achieved by applying active sensing method because of analytes concentration. Figure 5.15 illustrates these enhancement factors versus R6G concentration.

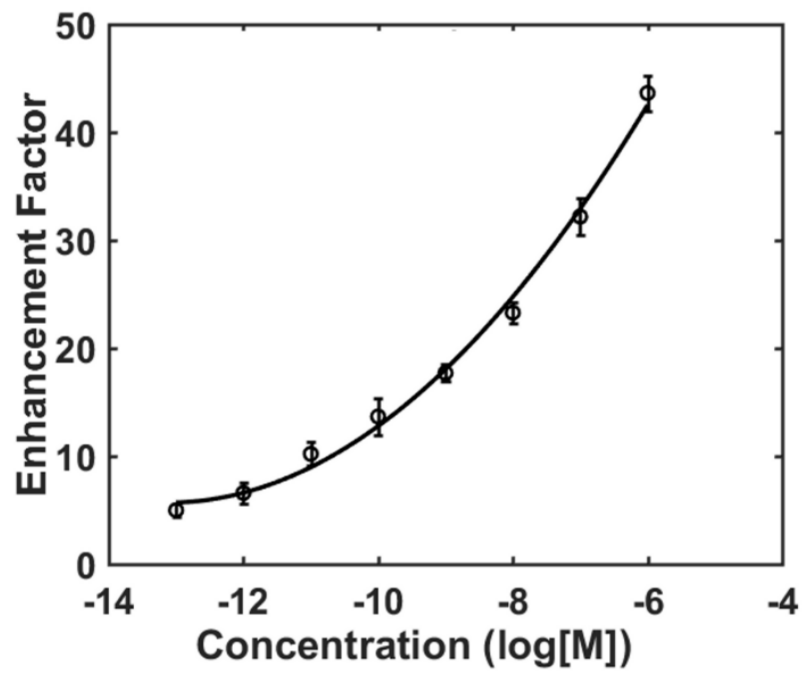

Figure 5.15 Enhancement factor of gold-nanoshell-structure as a function of R6G concentrations due to active sensing. ${ }^{23}$

It should be noted that, experimental data are fitted by the following function: 


$$
y=0.72 x^{2}+19.02 x+130.58 \quad \text { with } \mathrm{R}^{2}=0.995 .
$$

Here, $x$ is the $\log$ of concentration of R6G and $y$ is the enhancement factor of goldnanoshell-structure due to analytes concentration through active sensing of analytes.

According to Figure 5.15 an enhancement factor of $5 \pm 0.6$ due to analytes concentration is achieved at a concentration as low as $100 \mathrm{fM}$. It should be mentioned here that, enhancement factor because of analytes concentration through active sensing of analytes not only depends on the concentration of analytes but also depends on the type of nanogaprich-structures. For example, enhancement factor of $43 \pm 1.6$ is achieved in case of $1 \mu \mathrm{M}$ R6G on gold-nanoshell-structure. On the other hand, for the same concentration of R6G this enhancement factor is $32 \pm 1.5$ for gold-nanosphere-structure.

\subsection{Comparison of two nanogap-rich structures}

Based on the data shown above, gold-nanoshell-structure demonstrates a better performance compared to the gold-nanosphere-structure. This better sensitivity of goldnanoshell-structure may be a result of the different electric field profiels of gold-nanoshellstructure and gold-nanosphere-structure as follows:

\subsubsection{Electric field profile of two nanogap-rich structures}

Electric field profile of an array of gold nanosphere and gold nanoshell (silica core) are depicted in Figure 5.16 (a, b). Electric field profiles are simulated by finite-difference-timedomain (FDTD) method. In the simulation, $785 \mathrm{~nm}$ laser wavelength is used and it is the same laser wavelength used to record the experimental SERS spectra of R6G.

In the simulation, gold nanosphere array (inset of Figure 5.16 a) and gold nanoshell array (inset of Figure $5.16 \mathrm{~b}$ ) are used in order to mimic the nanogap-rich-structures fabricated 
through OGMB. The maximum electric fields of a gold-nanosphere-structure is $14 \mathrm{~V} / \mathrm{m}$ and most of the hot-spots are located mainly between the gold nanospheres as shown in Figure 5.16 (a). On the other hand, the maximum electric field of a gold-nanoshell-structure is $57 \mathrm{~V} / \mathrm{m}$ and most of the hot-spots are located mainly on gold nanoshells (silica core) surface as depicted in Figure 5.16 (b).
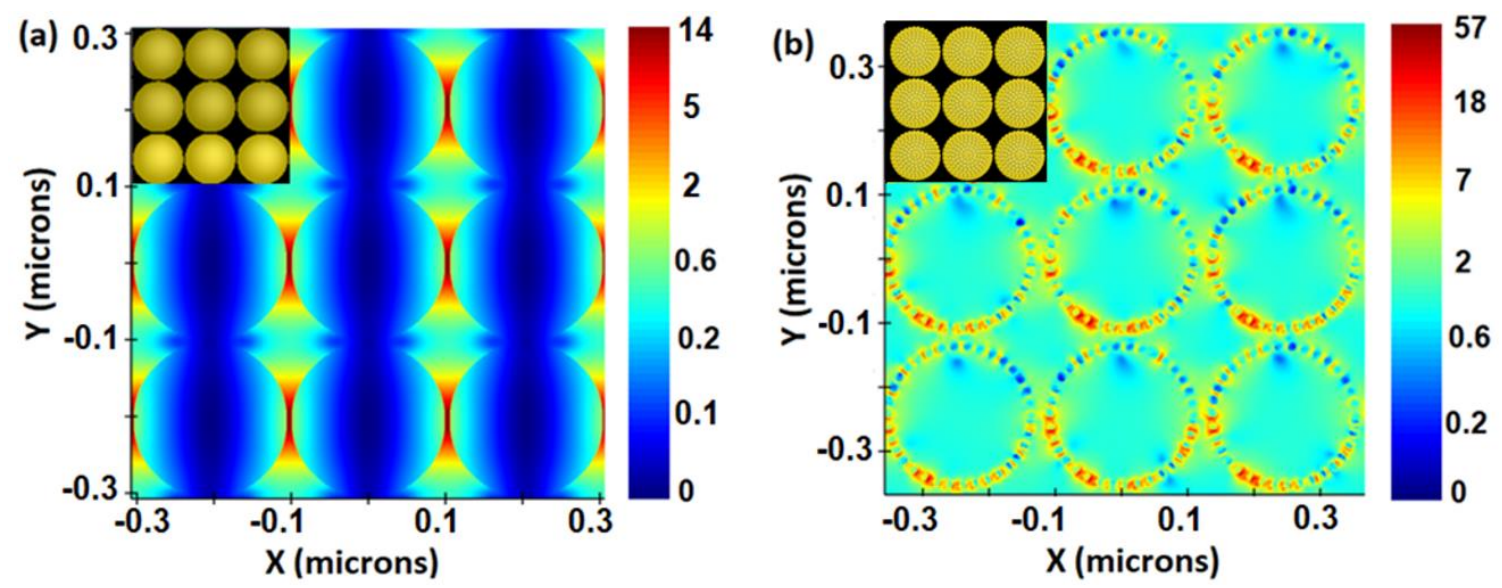

Figure 5.16 Simulated electric field profile of (a) gold nanosphere array (b) gold nanoshell (silica core) array at an exciting laser wavelength of $785 \mathrm{~nm}$. Inset: schematic structure of (a) gold nanosphere array (b) gold nanoshell (silica core) array. ${ }^{23}$

In addition, more hot-spots are provided by gold-nanoshell-structure than goldnanosphere-structure as depicted in Figure 5.16 (b) because of the structure of goldnanoshell (silica core). Each gold nanoshell (silica core) consists of a silica core with 200 $\mathrm{nm}$ diameter which is coated by $20 \mathrm{~nm}$ gold-nanospheres. The schematic structure of a gold nanoshell (silica core) array is shown in the inset of Figure 5.16 (b). Due to this structure of gold-nanoshell, most of the hot-spots or highest electric fields are observed between the gold nanospheres coating on the silica core of gold-nanoshell. 


\subsubsection{SERS spectra of R6G collected by two nanogap-rich structures}

As described above gold-nanoshell-structure provides more hot-spots and higher electric field compared to gold-nanosphere-structure. Because of higher electric fields and more hot-spots available on gold-nanoshell-structure compared to gold-nanosphere-structure, a higher Raman signal is observed in case of gold-nanoshell-structure since Raman signal is proportional to the fourth order magnitude of electric field. ${ }^{103}$

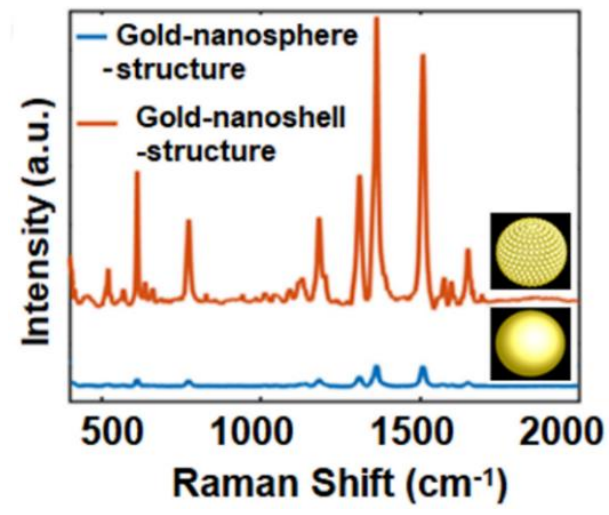

Figure 5.17 SERS spectrum of $1 \mu \mathrm{M}$ R6G recorded by gold-nanosphere-structure and gold-nanoshell-structure. Inset: schematic of a gold-nanosphere and gold-nanoshell. ${ }^{23}$

Figure 5.17 shows the SERS spectrum of $1 \mu \mathrm{M}$ R6G collected by gold-nanoshell-structure and gold-nanosphere-structure. SERS intensity of R6G at $1364 \mathrm{~cm}^{-1}$ in Figure 5.17 recorded by gold-nanoshell-structure is about 13 times higher than gold-nanospherestructure. Therefore, gold-nanoshell-structure provides better sensitivity in comparison to gold-nanosphere-structure.

\subsubsection{Uniformity of two nanogap-rich structures}

Uniformity of Raman signal is another important parameter for SERS measurement. The uniformity of two nanogap-rich-structures; gold-nanosphere-structure and gold-nanoshell- 
structure, is determined from the SERS spectra of R6G recorded from 15 random spots of two nanogap-rich-structures. Figure $5.18(\mathrm{a}, \mathrm{b})$ illustrates the histogram of SERS intensity at $1364 \mathrm{~cm}^{-1}$ for $100 \mathrm{pM} \mathrm{R6G}$ collected from gold-nanosphere-structure and goldnanoshell-structure respectively. It should be mentioned here that, variation of SERS intensity, $\Delta I$ with respect to average SERS intensity, $I_{\text {ave }}$ (as indicated by the red solid line in Figure 5.18) is calculated by the formula as stated below:

$$
\Delta I=\frac{\left|I_{m}-I_{\text {ave }}\right|}{I_{m}} \times 100 \%
$$

Here, $I_{m}=$ minimum or maximum SERS intensity.
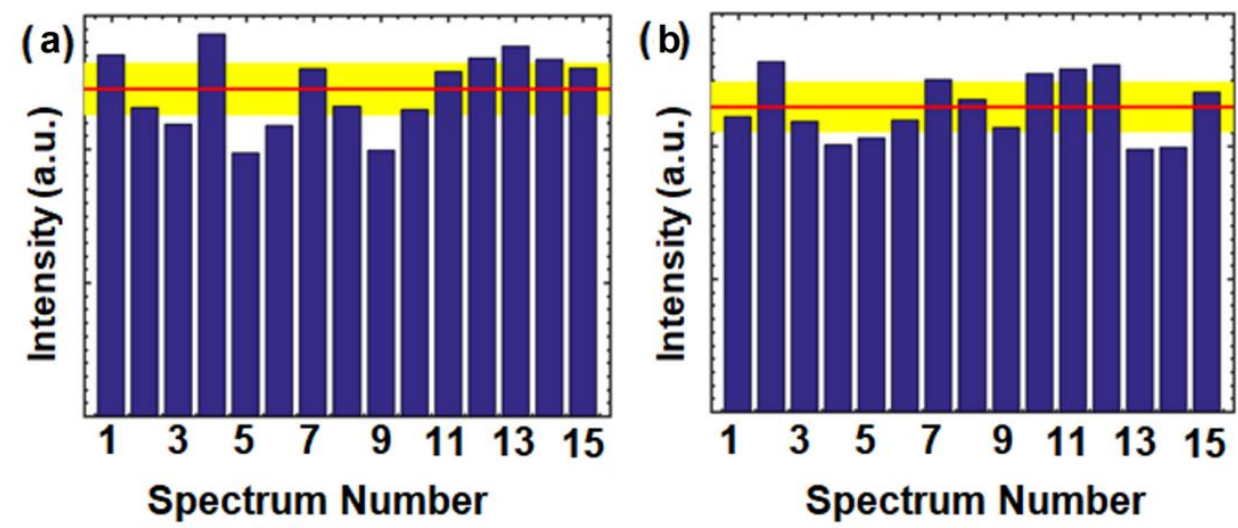

Figure 5.18 (a, b) Histogram of SERS intensity variation of $100 \mathrm{pM} \mathrm{R6G}$ at $1364 \mathrm{~cm}^{-1}$. SERS spectra of R6G were collected from 15 different spots of a (a) gold-nanospherestructure (b) gold-nanoshell-structure. ${ }^{23}$

Minimum and maximum SERS intensity variation are calculated as 5\% and $20 \%$ respectively for gold-nanosphere-structure according to the above formula. In case of goldnanoshell-structure, the minimum and maximum variation of SERS intensity are 3\% and $15 \%$ respectively. Here, variation in SERS intensity happens due to different number of nanogaps which exist within the spot of Raman exciting laser as determined by OGMBbased nanogap-rich-structures fabrication process. Large SERS intensity variation is not 
observed from our experimental data which demonstrates a very good uniformity of our OGMB-based fabricated nanogap-rich-structures.

\subsection{Active sensing of Malachite Green (MG)}

In this work, to demonstrate OGMB-assisted active sensing method, R6G molecules are used since R6G is generally used as a benchmark for Raman spectroscopy as reported in the literature. ${ }^{104,105}$ However, we have also tested OGMB-assisted nanogap-rich-structure for the detection of other molecule to demonstrate OGMB-based active sensing method. Based on the above discussion, the sensitivity of gold-nanoshell-structure is better than gold-nanosphere-structure. Therefore, we have used gold-nanoshell-structure for the detection of other chemical known as malachite green (MG) fungicide. The fungicide, $\mathrm{MG}$ is an organic dye and it is widely used in the aquaculture industry, fresh water aquaria and fish farms to kill bacteria, fungus and toxins because of its effective fungicide effect.
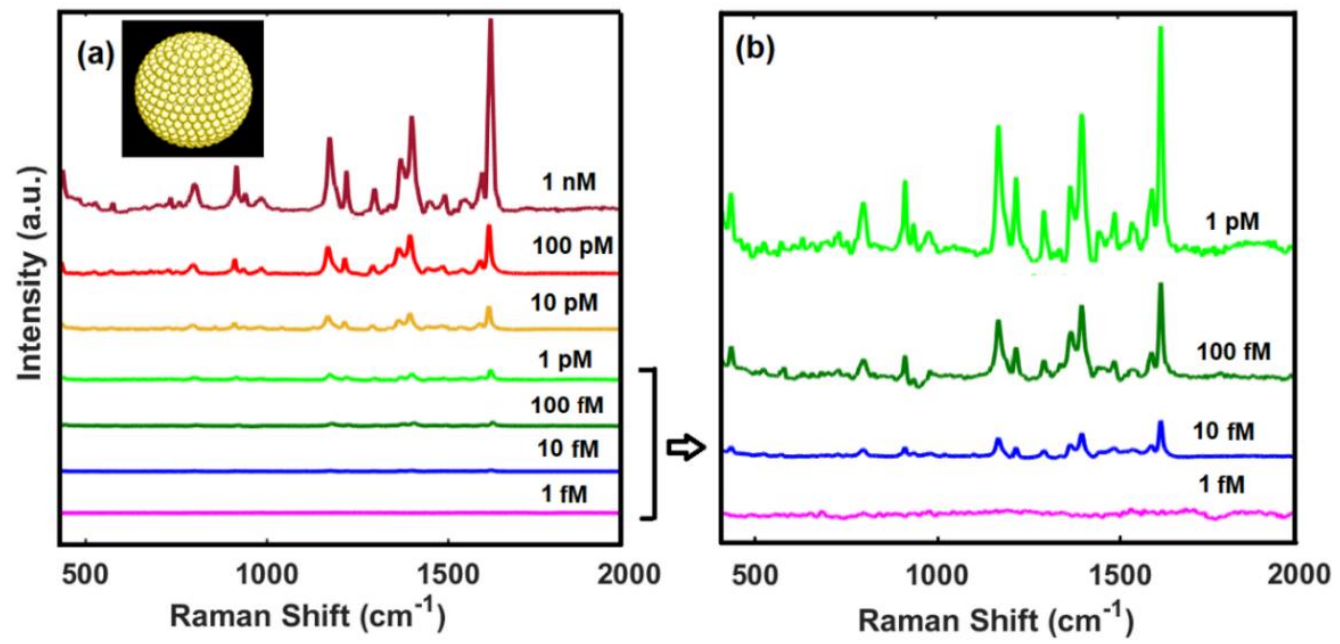

Figure 5.19 (a) SERS spectra of different concentrations (from $1 \mathrm{nM}$ to $1 \mathrm{fM}$ ) of MG collected by gold-nanoshell-structure through active sensing method. Inset: schematic of a gold-nanoshell used to fabricate gold-nanoshell-structure. (b) magnified image of SERS spectra of lower concentrations (from $1 \mathrm{pM}$ to $1 \mathrm{fM}$ ) of MG marked in (a) by an arrow. ${ }^{23}$ 
The fungicide, $M G$ is an organic dye and it is widely used in the aquaculture industry, fresh water aquaria and fish farms to kill bacteria, fungus and toxins because of its effective fungicide effect. However, use of MG is controversial and also banned in some of the countries of the world because of its hazardous and genotoxic nature. ${ }^{67}$

SERS spectra of different concentrations of MG collected through gold-nanoshell-structure by applying active sensing method is depicted in Figure 5.19 (a). SERS spectra of MG at lower concentrations are also shown in Figure 5.19 (b). According to Figure 5.19, MG is detectable up to $10 \mathrm{fM}$ concentration by our OGMB-assisted gold-nanoshell-structure through active sensing method.

In the SERS spectra, following characteristic Raman peaks of $\mathrm{MG}$ are detected: $\mathrm{C}-\mathrm{C}$ stretching and N-C bonding at $1619 \mathrm{~cm}^{-1}, \mathrm{~N}$-phenyl stretching at $1402 \mathrm{~cm}^{-1}, \mathrm{~N}-\mathrm{C}$ stretching at $1369 \mathrm{~cm}^{-1}, \mathrm{C}-\mathrm{H}$ in-plane bending at $1298 \mathrm{~cm}^{-1}$ and $1174 \mathrm{~cm}^{-1}, \mathrm{C}-\mathrm{H}$ out-of-plane bending at $918 \mathrm{~cm}^{-1}$ and these peaks are closely matches with the Raman peaks of MG reported in the literature. ${ }^{67,106}$

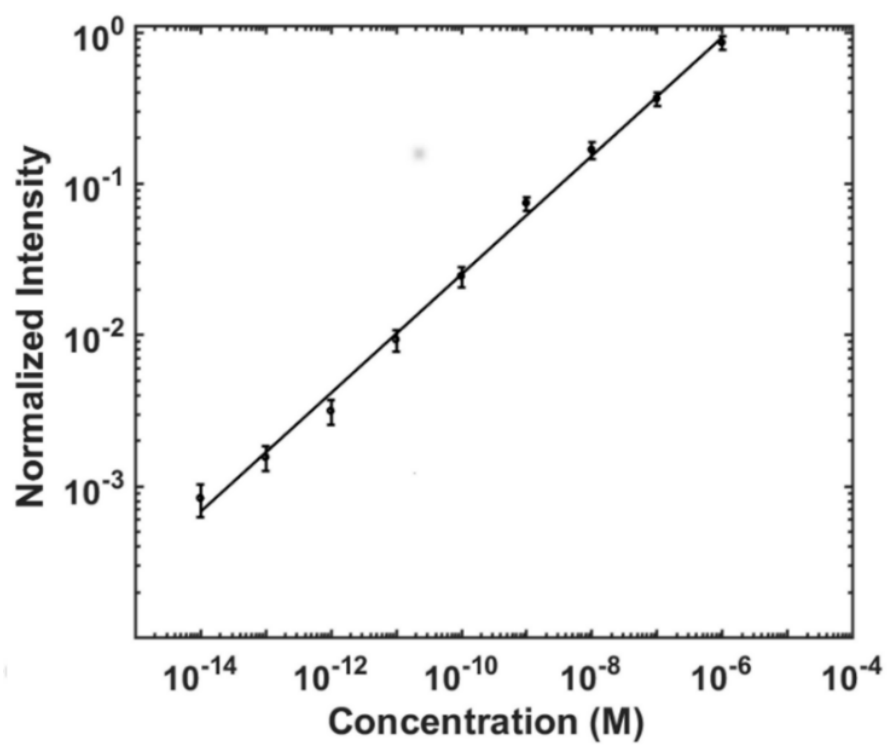

Figure 5.20 Normalized SERS intensity of MG at $1,619 \mathrm{~cm}^{-1}$ as a function of MG concentration by applying active sensing method. ${ }^{23}$ 
Figure 5.20 depicts the normalized SERS intensity of MG at $1619 \mathrm{~cm}^{-1}$ versus concentration of MG. The experimental data (circles) in Figure 5.20 are fitted by the following equation:

$$
\log _{10}(y)=0.39 \log _{10}(x)+2.31 \quad \text { with } \quad \mathrm{R}^{2}=0.996
$$

Where, $x$ is the concentration of MG and y represents normalized SERS intensity of MG at $1619 \mathrm{~cm}^{-1}$ by applying active sensing method.

It should be noted that, all the SERS spectra of R6G and MG at different concentrations, are reported in this work after performing the baseline correction from the raw SERS spectra of R6G and MG, respectively. Through the baseline correction we can avoid large flurescent background signal of R6G and MG recorded by $785 \mathrm{~nm}$ Raman system.
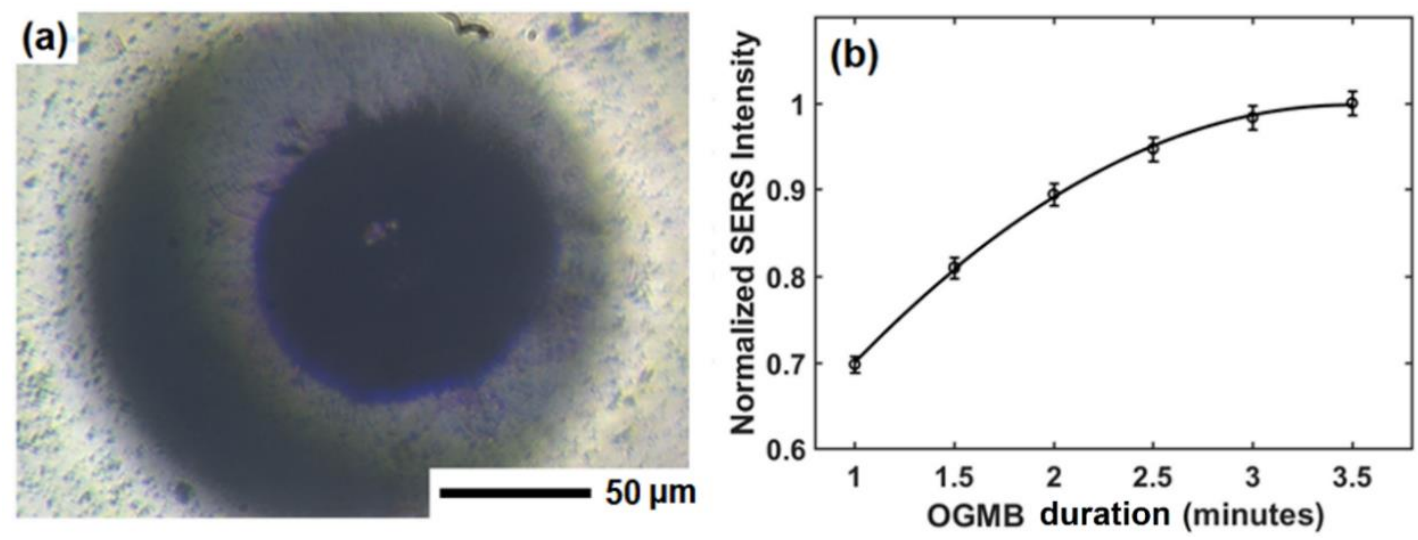

Figure 5.21 (a) An OGMB which is generated on gold-nanoshell-structure for active sensing of analytes, R6G. (b) normalized SERS intensity of R6G versus the duration of OGMB generation during the active analytes sensing. ${ }^{23}$

The size of OGMB during the active sensing of analytes was about $175 \pm 2 \mu \mathrm{m}$ as shown in Figure 5.21 and duration of OGMB generation from analytes was about 2 minutes for all 
of the experimental data reported in this research. The laser intensity was $10 \mathrm{~mW} / \mu \mathrm{m}^{2}$ for the generation of OGMB from analytes on the nanogap-rich-structures during the active sensing of analytes. This intensity which is needed to generate OGMB from analytes solution during active sensing is much lower than $34 \mathrm{~mW} / \mu \mathrm{m}^{2}$ laser intensity needed for the fabrication of nanogap-rich structures. The reason for this reduced laser intensity used during the active sensing of analytes is the enhanced heating effect produced from gold nanoparticles, as studied and reported in the literature. ${ }^{107-109}$

According to Figure 5.21 (b), SERS intensity increases with the increasing of OGMB generation time during active analytes sensing until SERS signal saturates at around 3.5 minutes after OGMB generation from analytes. Above experimental results demonstrates that, the detection limits of gold-nanosphere-structure through the passive and active sensing method are $10 \mathrm{pM}$ and $1 \mathrm{pM}$ respectively. However, the detection limit has been further improved through the use of gold-nanoshell-structure. The detection limits of goldnanoshells-structure through the passive and active sensing method are $100 \mathrm{fM}$ and $10 \mathrm{fM}$, respectively. Therefore, the sensitivity and detection limit of a nanogap-rich-structure can be improved one order of magnitude by applying OGMB-based active sensing method compared to that with a passive sensing method. Therefore, our OGMB-assisted active sensing method can be adopted to any other sensing system to overcome the diffusion limit of conventional passive sensing methods. 


\section{CHAPTER 6}

\section{NANOGAP-RICH STRUCTURE FOR BIOSENSING APPLICATION}

In chapter 5 we have explained the performance of two nanogap-rich structures, goldnanosphere-structure and gold-nanoshell-structure for active chemical sensing application. Based on the experimental results reported in chapter 5, the sensitivity of gold-nanoshellstructure is better than gold-nanosphere-structure. Therefore, in this chapter we have used gold-nanoshell-structure for biosensing application. In addition, we have used another nanogap-rich-structure known as gold-nanostar-structure for biosensing application and observe which nanogap-rich-structure provide better sensitivity. In this chapter, we are going to discuss ultrasensitive bio-sensing application of nanogap-rich structures through the SERS detection of food-borne bacteria, Listeria. The performance of two types of nanogap-rich structures; gold-nanoshells-structure and gold-nanostars-structure for ultrasensitive biosensing application is explained in detail in the following section.

\subsection{Listeria monocytogenes detection by nanogap-rich structures}

In this research we have used Listeria as a biological sample to demonstrate the biosensing application of our OGMB-assisted nanogap-rich structures. Listeria monocytogenes (Listeria) is a food-borne disease-causing bacterium which is responsible for listeriosis disease. ${ }^{110}$ People get sick through the consumption of foods that carries it. Once in the body, Listeria can travel through blood stream and produce toxins that can damage cells. The infection can be serious or even life-threatening, particularly for pregnant women and their expected babies, people with weak immune systems and seniors. ${ }^{110}$ Listeria is cultured at Dr. Sun's lab which is located in the department of Biology of University of Dayton. Two nanogap-rich structures known as gold-nanoshell-structure and gold-nanostar-structure 
were used for the detection of Listeria. The performance of these two nanogap-rich structures are described in the following section.

\subsubsection{Performance of gold-nanoshell-structure and gold-nanostar-structure}

The SERS spectra of Listeria collected by gold-nanoshell-structure and gold-nanostarstructure are shown in Figure $6.1(a, b)$. All the SERS spectra were recorded with the Raman spectrometer as introduced in Chapter 5. During the recording of the SERS spectra of Listeria, laser power of the Raman spectrometer was kept at 10\% of the maximum laser power, which was $30 \mathrm{~mW}$ laser power, and the acquisition time was 10 seconds.
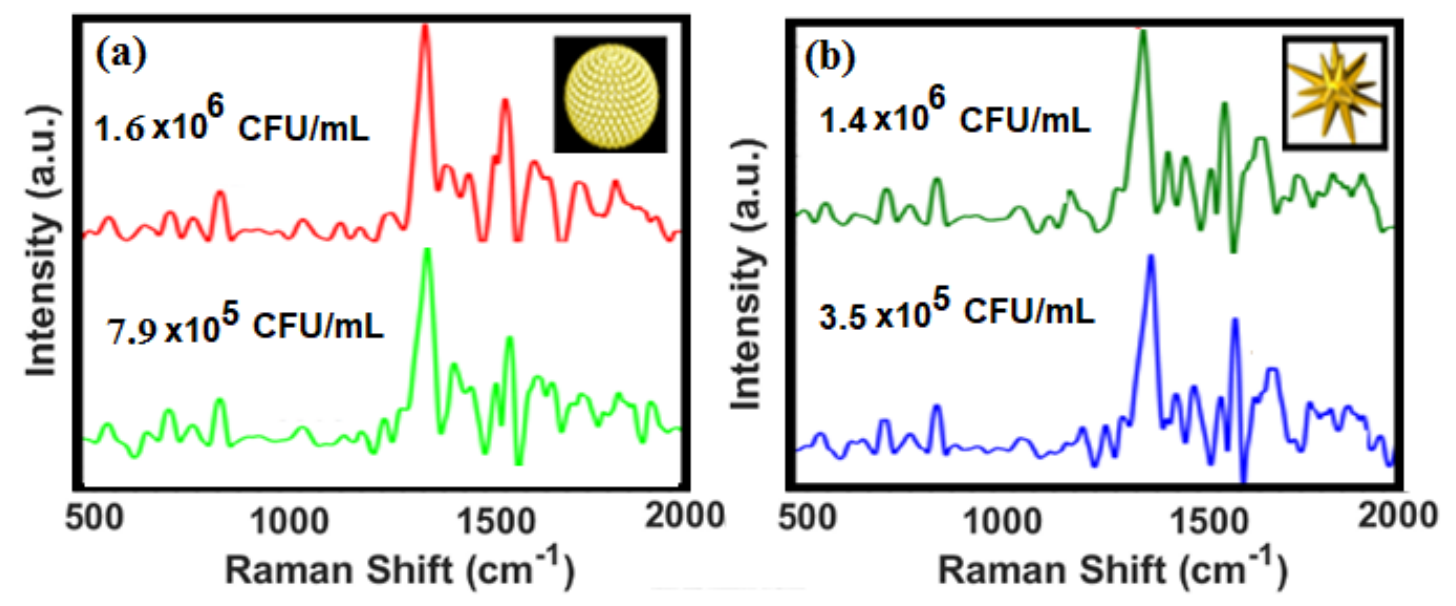

Figure 6.1 SERS spectra of different concentration of Listeria collected by (a) goldnanoshell structure (b) gold-nanostar-structure respectively. Inset: schematic of (a) goldnanoshell (b) gold-nanostar used for the fabrication of gold-nanoshell-structure and goldnanostar-structure. ${ }^{11}$

In this experiment, we have successfully detected Listeria upto $7.9 \times 10^{5}$ colony forming unit $/ \mathrm{mL}(\mathrm{CFU} / \mathrm{mL})$ and $3.5 \times 10^{5}$ colony forming unit $/ \mathrm{mL}(\mathrm{CFU} / \mathrm{mL})$ through goldnanoshell-structure and gold-nanostar-structure respectively, as shown in Figure $6.1(\mathrm{a}, \mathrm{b})$. 
In the SERS spectra, following characteristic Raman peaks of Listeria monocytogenes (Listeria) are detected: deformational vibration of adenine at $740 \mathrm{~cm}^{-1}$, cytosine or uracil (nucleic acids) at $798 \mathrm{~cm}^{-1}$, symmetric ring breathing mode of phenylalanine at $1000 \mathrm{~cm}^{-1}$, $\mathrm{CH}_{3} \mathrm{CH}_{2}$ vibrational modes at $1345 \mathrm{~cm}^{-1}$, amide $\mathrm{I}$ band due to $\mathrm{C}=\mathrm{O}$ stretching vibrations at $1650 \mathrm{~cm}^{-1}$. These peaks are closely matches with the Raman peaks of Listeria monocytogenes reported in the literature. ${ }^{112}$

Experimental results reported in Figure 6.1 demonstrates that, gold-nanostar-structure shows a higer sensitivity compared to gold-nanoshell-structure through the detection of Listeria upto $3.5 \times 10^{5}$ colony forming unit $/ \mathrm{mL}(\mathrm{CFU} / \mathrm{mL})$ concentration. Experimental sensing conditions, experimental error, SERS enhancement factor of nanogap-rich structure are described in the following section.

\subsubsection{Experimental sensing conditions}

Passive sensing method was applied during the SERS spectra recording of Listeria through OGMB-assisted fabricated nanogap-rich structures.

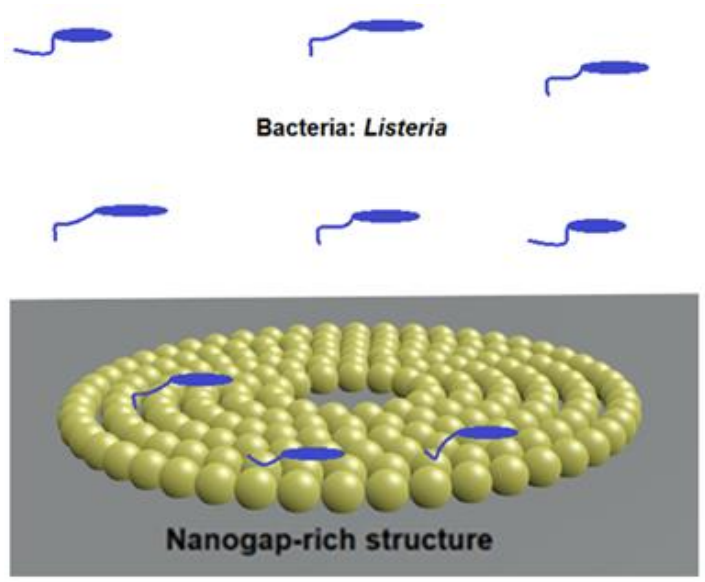

Figure 6.2 Schematic of free diffusion of Listeria towards nanogap-rich structure which demonstrates passive sensing of Listeria. 
As described in the previous chapters, here passive sensing means analytes, Listeria is freely difussed towards the nanogap-rich structures as shematically shown in Figure 6.2. From the above experimental results as shown in section 6.1.1, gold-nanostar-structure shows better sensitivity compared to gold-nanoshell-structure in Listeria detection. Figure 6.3 depicts the normalized SERS intensity of Listeria at $1345 \mathrm{~cm}^{-1}$ versus concentration of Listeria. SERS spectra of Listeria were collected by gold-nanostar-structure. The experimental data (circles) in Figure 6.3 are fitted by the following equation:

$$
\log _{10}(y)=0.77 \log _{10}(x)+2.4 \quad \text { with } \mathrm{R}^{2}=0.99
$$

Here, $x$ is the concentration of Listeria, and $y$ is SERS intensity of Listeria at $1345 \mathrm{~cm}^{-1}$.

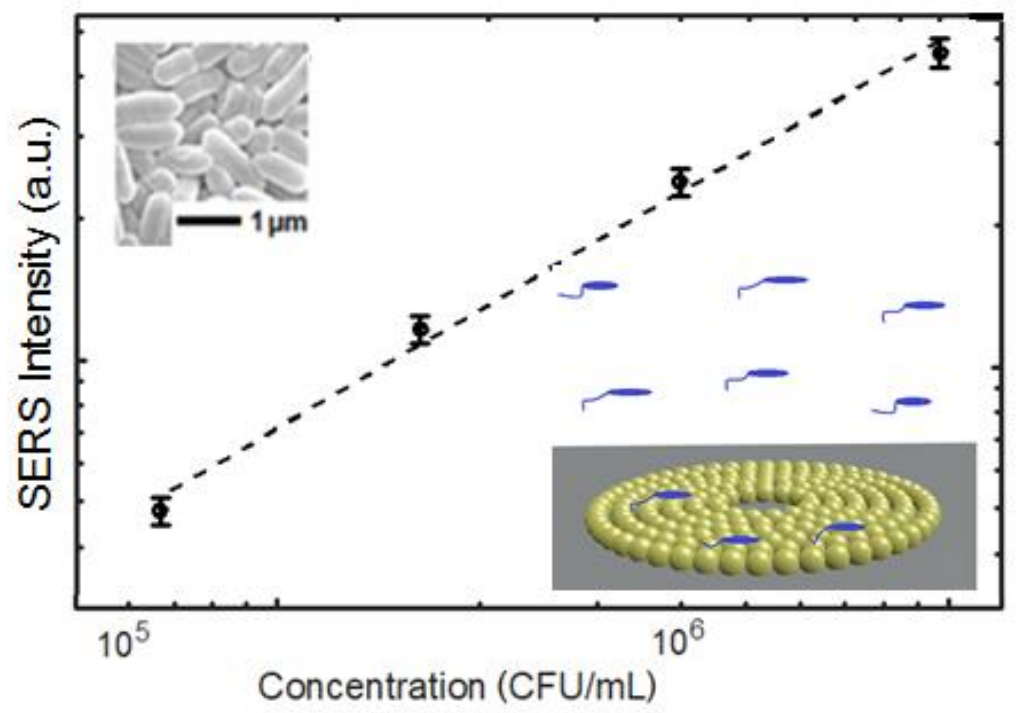

Figure 6.3 Normalized SERS intensity (with error bars) of Listeria at $1345 \mathrm{~cm}^{-1}$ as a function of Listeria concentration. Inset (bottom): schematic of passive sensing method shows free diffusion of Listeria towards nanogap-rich structure. Inset (top): Scanning Electron Microscope (SEM) image of Listeria monocytogenes (Listeria) which was detected by gold-nanostar-structure.

Experimental error bars in Figure 6.3, are calculated from four sets of SERS spectra of Listeria recorded at four different spots of gold-nanostar-structure. 


\subsection{SERS enhancement factor of gold-nanostar-structure}

The average SERS enhancement factor of gold-nanostar-structure was calculated for the passive sensing of Listeria. This enhancement factors (EF) was calculated through the comparison of the SERS signals of Listeria at $3.1 \times 10^{5}$ colony forming unit $/ \mathrm{mL}(\mathrm{CFU} / \mathrm{mL})$ concentration collected by gold-nanostar-structure and Raman signals of $1.6 \times 10^{8}$ colony forming unit $/ \mathrm{mL}(\mathrm{CFU} / \mathrm{mL})$ of Listeria recorded in absence of gold-nanostar-structure according to the formula as stated below: 100

$$
E F=\frac{I_{S E R S}}{I_{R S}} \times \frac{C_{R S}}{C_{S E R S}}
$$

Where, ISERS $=$ SERS intensities of Listeria at $1345 \mathrm{~cm}^{-1}$ on gold-nanostar-structure.

$I_{R S}=$ Raman intensities of Listeria at $1345 \mathrm{~cm}^{-1}$ in absence of gold-nanostar-structure.

$C_{R S}=1.6 \times 10^{8} \mathrm{CFU} / \mathrm{mL}$ concentration of Listeria for normal Raman measurement.

$C_{S E R S}=3.1 \times 10^{5} \mathrm{CFU} / \mathrm{mL}$ concentration of Listeria for SERS measurement.

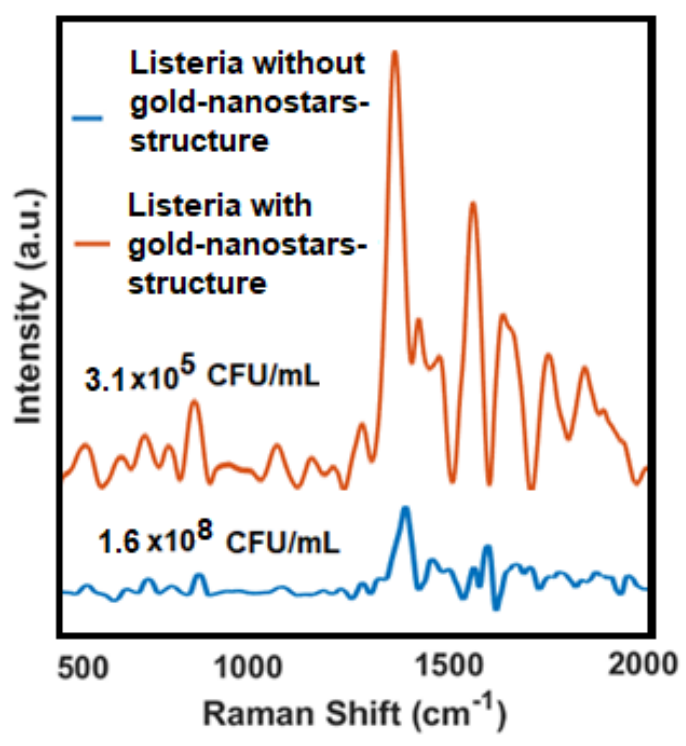

Figure 6.4 Raman intensity of Listeria at $1345 \mathrm{~cm}^{-1}$ without gold-nanostar-structure (blue curve) and with gold-nanostar-structure (red curve). 
Figure 6.4 illustrates SERS intensity of $3.1 \times 10^{5} \mathrm{CFU} / \mathrm{mL}$ concentration of Listeria at 1345 $\mathrm{cm}^{-1}$ with gold-nanostar-structure (red curve) and the corresponding Raman intensity of $1.6 \times 10^{8} \mathrm{CFU} / \mathrm{mL}$ concentration of Listeria at $1345 \mathrm{~cm}^{-1}$ without the gold-nanostarstructure (blue curve).

Average SERS enhancement factor (EF) of gold-nanostar-structure is $2.1 \times 10^{4}$ which was calculated from four sets of experimental data according to the above formula. The SERS enhancement factor of gold-nanostar-structure is comparable to the SERS enancement factor for many conventional SERS substrates used for bacteria detection using label-free probes reported in the literature. ${ }^{113,114}$

The size of Listeria used in this experiment is around $1 \mu \mathrm{m}$ as shown in the scanning electron microscope (SEM) image of Listeria monocytogenes (inset of Figure 6.3). Therefore, the size of Listeria used in this experiment is much larger than the size of nanogaps in nanogap-rich structure. Because of this larger size of Listeria, enhancement in SERS signal of Listeria would not happen in this experiment due to the plasmonic resonance present between the nanogaps of OGMB-based nanogap-rich structure.

Rather, enhancement in the SERS signal of Listeria happens due to the interaction of Listeria near the surface of nanogap-rich structure within the evanescent field. It is reported that, biosensors that works based on evanescent waves often have the high sensitivities for biological sample detection due to the interaction of molecules near the sensor surface within the evanescent field. ${ }^{115}$ SERS detection due to the evanescent field is also studied by several other groups. ${ }^{116-118}$ 


\subsection{Limitation of active sensing method for biosensing application}

In this research, we have applied passive sensing method to demonstrate OGMB-based nanogap-rich structure for biosensing application.

We did not apply active sensing method for biosensing application, since in order to apply OGMB-based active biosensing we need to generate OGMB from Listeria solution by using laser heating. However, biological objects for example proteins or bacteria might get

denatured at high temperature ${ }^{119}$ due to laser heating, which results in some changes in their Raman spectrum. Therefore, laser heating of Listeria could kill Listeria or the properties of Listeria might change. Due to this limitation, we did not apply OGMB-based active sensing method for Listeria detection. However, OGMB-based active sensing method can be applied for the detection of biological samples through the proper control of certain active sensing parameters. For example, laser intensity can be kept lower during the generation of OGMB from bacteria sample to apply active biosensing. Through the use of lower laser intensity we can avoid denaturation of bacteria which happens at high temperature. Therefore, in order to apply active sensing for biological samples, several active sensing parameters such as laser intensity or temperature, laser exposure time need to be controlled. 


\section{CHAPTER 7}

\section{REAL TIME ACTIVE SENSING OF ANALYTES BY OGMB-ASSISTED SERS}

The concentration of analytes towards nanogap-rich structures for active analytes sensing and SERS measurement of analytes are separated as reported in the previous chapters. For example, a nanogap-rich structure is first fabricated through an OGMB. Then analytes are guided and concentrated towards the nanogap-rich structures by generating another OGMB from analytes for active sensing. SERS measurements of analytes are performed after analytes are concentrated on the nanogap-rich structures through OGMB. However, we can combine these two processes to apply real time active analytes sensing. Here, real time active sensing of analytes means, SERS measuments of analytes are performed while analytes are concentrated on the nanogap-rich structures through an OGMB. Real-time active sensing of analytes is crucial for several biomedical applications through the rapid detection of hazardous chemicals and biological samples. In this chapter, we will describe the concept of real-time active sensing of analytes. We have performed some preliminary works based on this idea which are explained in the following section.

\subsection{Fabrication of nanogap-rich structures}

For this experiment, instead of a planner glass substrate we have used optical fiber probe as a substrate. Gold nanoshells (silica core) with $240 \mathrm{~nm}$ diameter consists of $200 \mathrm{~nm}$ silica core coated with $20 \mathrm{~nm}$ gold nanospheres from NanoComposix were used as nanoparticles in this experiment. FP200ERT- 0.50 NA, diameter $200 \mu \mathrm{m}$ core multimode fiber was used as a substrate. Figure 7.1 represents the schematic of experimental setup to develop an OGMB assisted SERS on an optical fiber probe. For this experiment, a near infra-red 
continuous-wave (cw) laser having wavelength $1064 \mathrm{~nm}$ was used as a heating laser. The laser beam was collimated and then expanded by a beam expander through the combination of two lenses, $\mathrm{L}_{1}$ and $\mathrm{L}_{2}$ and then focused on a substrate by a $40 \times$ microscope objective lens, $\mathrm{O}_{1}$, having numerical aperture, $\mathrm{NA}=0.65$. A beam splitter (BS) picked up a fraction of laser power and it was measured by a power meter (PM). The laser power was adjusted through a variable neutral density (ND) filter.
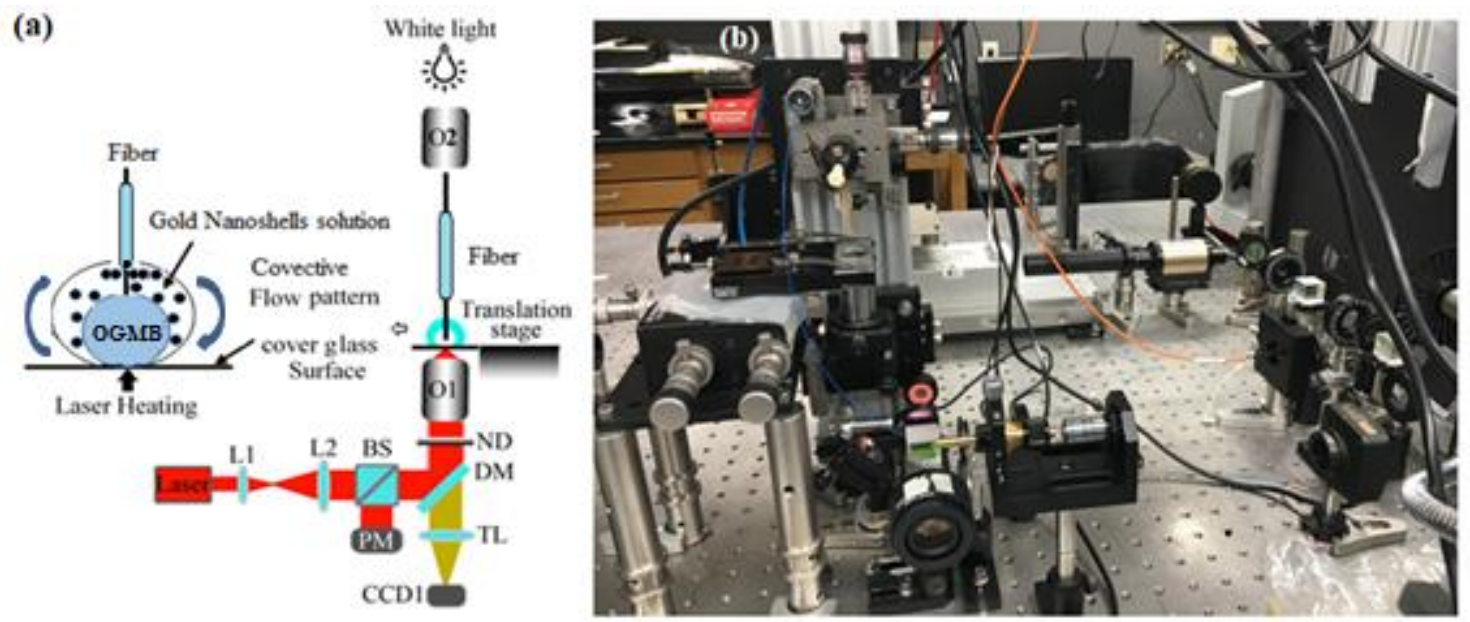

Figure 7.1 (a) Schematic of experimental setup. L: lens; BS: beam splitter; DM: dichroic mirror; O: objective lens; TL: tube lens; CCD: charge coupled device; ND: variable neutral density filter; PM: power meter. Inset: schematic of OGMB formation and gold nanoshells deposition on optical fiber substrate through strong Marangoni convective flow associated with OGMB. (b) real experimental setup at Nanophotonic and Nano-manufacturing (NPNM) lab led by Dr. Zhao.

A white-light source was used to illuminate the substrate and it was then imaged on a charge-coupled device (CCD) through O1 and a tube lens (TL). The purpose of using a dichroic mirror (DM) in this setup is to transmit the white light but reflect the infrared laser beam. In order to enhance the opto-thermal effect as well as to facilitate the imaging of scanning electron microscope (SEM), a 10-nm-thick gold film was deposited on the tip of optical fiber core by a sputter coater (Denton desk II) prior to generating OGMB. However, 
depositing gold film on the optical fiber core is not a pre-requirement for generating OGMB in this experiment. It is already described in chapter 4 of this dissertation, OGMB can be generated from nanoparticles solution on a glass coverslip substrate both with and without gold film. Nevertheless, higher laser intensity is needed to generate OGMB and deposit nanoparticles on the substrate. Effect of gold film for the generation of OGMB is reported in one of our publications in Optics Letters. ${ }^{22}$

For OGMB generation through laser heating, at first one droplet of nanoparticles (gold nanoshells) solution was placed on a glass coverslip substrate. Fiber tip was then immersed into the droplet of gold nanoshells solution. Laser beam was focused on the optical fiber tip. Since the fiber tip was immersed into the gold nanoshells solution, eventually goldcoated optical fiber tip along with gold nanoshells were heated by the focused laser beam and an OGMB was generated on the optical fiber core.

Through the generation of OGMB a strong Marangoni convective flow has been induced that helps to rapidly deposit gold nanoshells on the core of optical fiber. Schematic of OGMB formation and gold nanoshells deposition on optical fiber substrate through strong Marangoni convective flow is illustrated in the inset of Figure 7.1.

Through the accumulation of gold nanoshells on optical fiber, a nanogap-rich structure has been developed. Repeating the same procdure of OGMB generaton through laser heating as described above, several nanogap-rich structures have been fabricated on the core of optical-fiber as shown in Figure 7.2 (a). Figure 7.2 (b) shows the SEM image of the corresponding area which is marked in Figure 7.2 (a) by a white box. We refer the nanogap-rich structures on the optical fiber core as gold-nanoshells-nanogap-rich structure since the nanogaprich structure are fabricated from gold nanoshells. The gold-nanoshells-nanogap-rich-structures on 
optical fiber core serve as a SERS substrate due to the plasmonic resonance ${ }^{24-26}$ of nanogaprich structures.
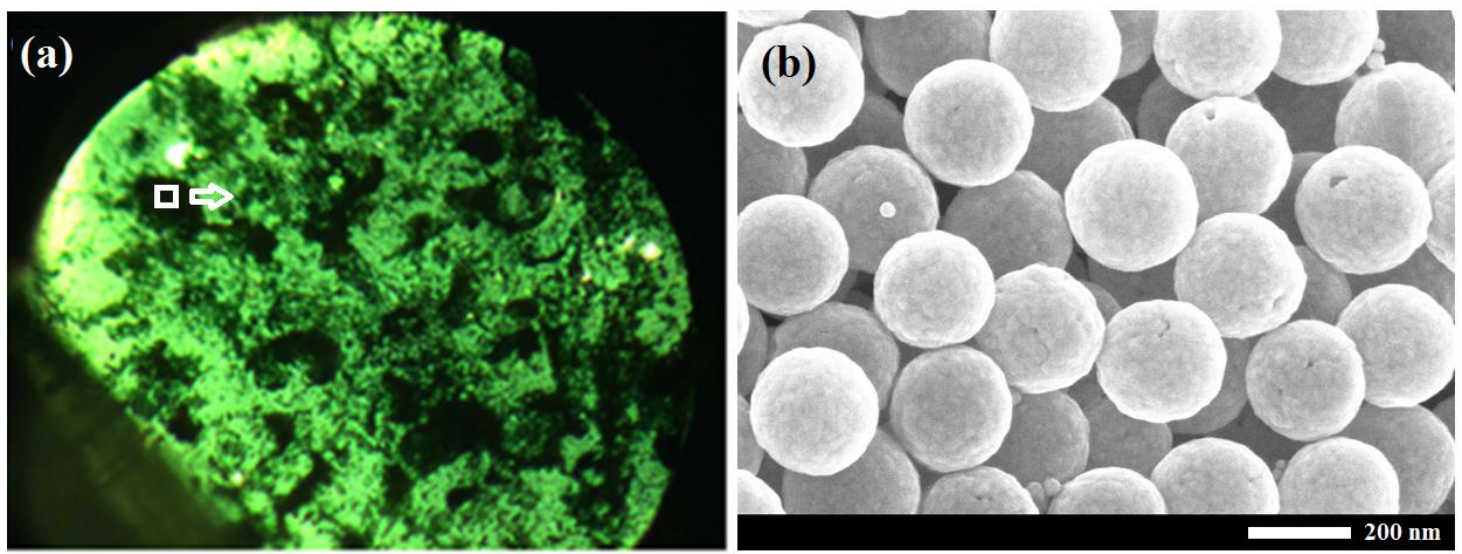

Figure 7.2 (a) Optical image of an optical fiber core with several nanogap-rich-structures fabricated by OGMB (b) SEM image of the corresponding area which is marked in (a) by a white box.

Printing of mettalic nanoparticles on an optical fiber through an OGMB also serve as a SERS substrate which is reported in one of our recent publications ${ }^{120}$. However, OGMBbased SERS on an optical fiber reported in reference ${ }^{120}$ is used only for passive chemical sening application and therefore it is still a diffusion limited method. This work is different from the work that reported in both references ${ }^{23,120}$ bcause it aims for the real-time active sensing of analytes.

\subsection{Real time active sensing of analytes}

Rhodamine 6G (R6G) molecules and Urea were detected through this OGMB assisted SERS on an optical fiber. After the fabrication of nanogap-rich structures from nanoparticles solution, chemical samples were attached on the nanogap-rich structures and laser beam was focused on the optical fiber tip to generate another OGMB from chemical sample. Due to strong Marangoni convective flow associated with an OGMB, chemical 
samples were accumulated on the nanogap-rich structure and therefore locally increase the concentration of chemical on the nanogap-rich structure for active chemical sensing. In order to apply real-time active chemical sensing, an OGMB was generated from the chemical samples to concentrate chemicals on optical fiber SERS substrate. SERS measuments of chemicals were performed while analytes are concentrated on the SERS substrate through an OGMB generated from the analytes. As a chemical, R6G and Urea were used in this experiment.

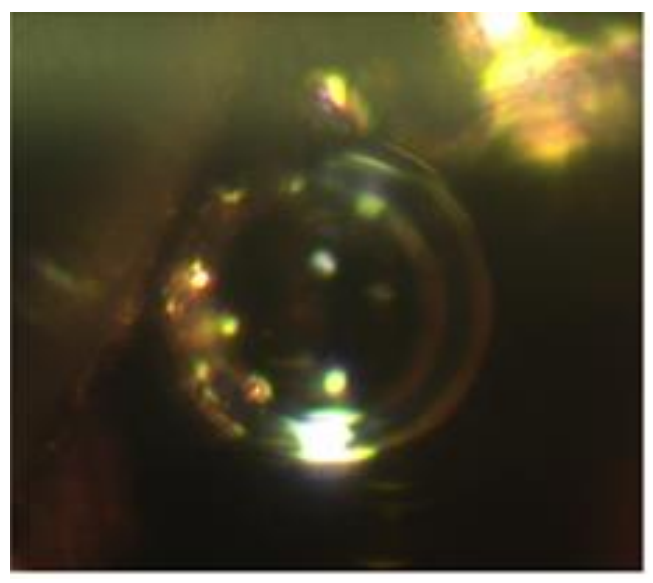

Figure 7.3 An OGMB generated from R6G molecules during the SERS detection of R6G.

An OGMB was generated from R6G and Urea solution during the SERS detection period of R6G and Urea. One such an OGMB generated from R6G molecules is depicted in Figure 7.3. This OGMB generation during the SERS detection period of R6G and Urea helps to locally increase the concentration of R6G or Urea on nanogap-rich structure for real time active sensing of R6G or Urea. SERS detection results of R6G and Urea are illustrated in Figure 7.4 (a, b). $50 \mathrm{mM}$ Urea and $50 \mathrm{mM}$ R6G were successfully detected by OGMBassisted gold-nanoshell-nanogap-rich structure fabricated on optical fiber core. SERS spectra of Urea and R6G were recorded in this experiment by $1064 \mathrm{~nm}$ Raman spectrometer from Ocean Optics. Therefore, for real time active sensing of analytes the 
same $1064 \mathrm{~nm}$ laser was used for the fabrication of nanogap-rich structure and SERS measurement which simplifies the overall experimental setup. The laser power was kept at $900 \mathrm{~mW}$ and integration time for the SERS measurements was 10 seconds.
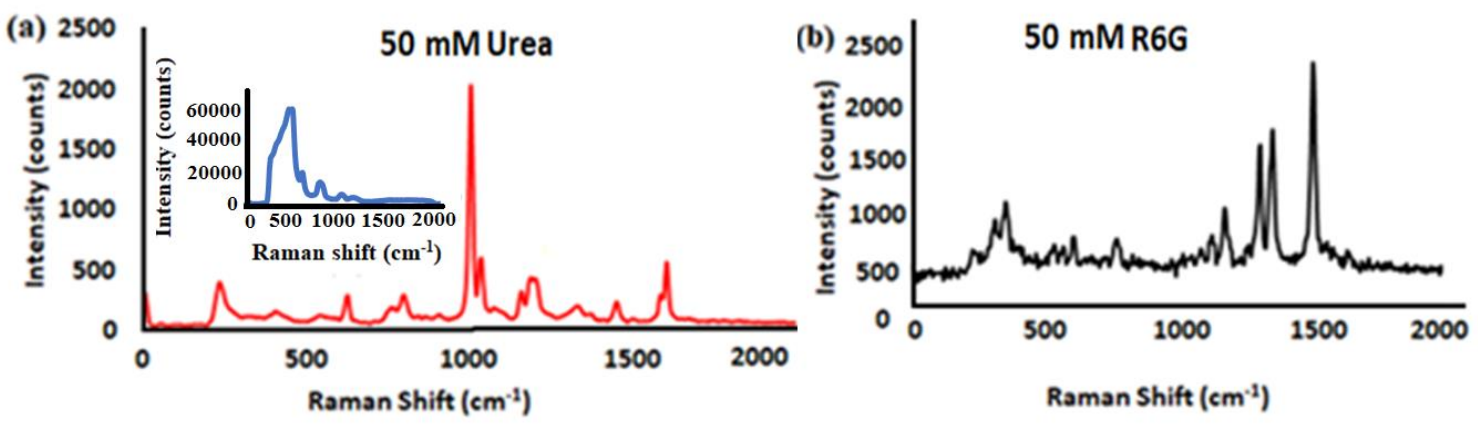

Figure 7.4 SERS spectra of (a) $50 \mathrm{mM}$ Urea (b) $50 \mathrm{mM}$ R6G collected by OGMB-assisted gold nanoshell-structure fabricated on optical fiber core after removing the background Raman signal of optical fiber as depicted in the inset of (a).

One important advantage of 1064-nm Raman system is, it can significantly reduce the fluorescence background of analytes. Therefore, no base line correction or any other further processing of raw Raman data is required. However, Raman signal detected by $1064 \mathrm{~nm}$ Raman system is also weaker than that from 785-nm Raman system because the Raman intensity is inversely proportional to the fourth power of the laser wavelength. ${ }^{121}$ The SERS spectra of R6G and Urea shown in Figure 7.4 is obainted only after removing the background Raman signal of bare optical fiber as depicted in the inset of Figure 7.4 (a). No further processing of raw SERS data is required due to the less flurescent background signal of R6G and Urea detected by $1064 \mathrm{~nm}$ Raman system. However, these are just preliminary experimental results. Future work is needed to improve the detection limit of R6G and Urea and thus enable this real-time active analytes sensing for advanced chemical and biosensing application. 


\section{CHAPTER 8 \\ CONCLUSION AND FUTURE WORK}

In this chapter we will discuss how we have successfully met all the objectives of this research as mentioned in the 'objectives of this research' section of chapter 1. In this chapter, we will conclude our discussion through the main outcome from this work, application and future recommended work of this research.

\subsection{Conclusion}

In this work, an OGMB-based nanogap-rich structure has been fabricated under ambient condition to demonstrate active sensing of analytes. OGMB has been generated through the laser heating. Laser-based heating allows us to heat at the nanoscales which is challenging to achieve with other heat source. For example, illuminating gold nanoparticles with a laser allows us to confine the heating around the nanoparticles. In addition, heating efficiency can be remotely tunable with laser.

OGMB induces a strong Marangoni convective flow through which nanoparticles are attracted towards OGMB and rapidly deposited on the surface of substrate for rapid and cost-effective fabrication of nanogap-rich-structure under ambient condition. As a substrate, we have used simple glass coverslip substrate with and without gold film. As discussed in chapter 4 of this dissertation and also reported in one of our publications in Optics Letters, ${ }^{22}$ gold coating on glass coverslip is not a pre-requirement for the generation of OGMB and fabrication of nanogap-rich structure. In this work, nanogap-rich structures can be rapidly fabricated through OGMB on any kind of subtrate under ambient conditions. No special substrate or special vacuum environment is needed to fabricate the nanogap- 
rich structure. Therefore, OGMB-based fabrication of nanogap-rich structure applied in this research is a rapid and cost-effective fabrication method.

Three types of metallic nanoparticles: $200 \mathrm{~nm}$ gold nanospheres, $240 \mathrm{~nm}$ gold nanoshells consists of $200 \mathrm{~nm}$ silica core coated with $20 \mathrm{~nm}$ gold nanospheres and $100 \mathrm{~nm}$ gold nanostars are used to fabricate three types of nanogap-rich-structures under ambient condition known as gold-nanosphere-structure, gold-nanoshell-structure and goldnanostar-structure respectively. Fabricated nanogap-rich structures form many nanogaps which are ideal for SERS enhancement due to the plasmonic resonance ${ }^{24-26}$ of nanogaprich structure and thus OGMB-assisted nanogap-rich structures serve as SERS substrates. After the fabrication of nanogap-rich structures, we have attached analytes such as any chemical or biological samples on the nanogap-rich structure to develop an ultrasensitive chemical and biosensing platform. Then we have used this chemical and biosensing platform for active and ultrasensitive chemical and biosensing application through promising surface enhanced Raman spectroscopy (SERS) tool. As described in chapter 5 and also reported in our most recent publication in Nanoscale (2019), ${ }^{23}$ OGMB is not only used for rapid and affordable fabrication of nanogap-rich structure under ambient condition but also used to actively guide and concentrate analytes on the surface of nanogap-richstructure for active analytes sensing. OGMB-based active sensing method used in this research force the analytes towards the nanogap-rich structure for active diffusion. This can significantly improve the detection limit of nanogap-rich structure by locally increase the concentration of analytes on the nanogap-rich structure and thus overcome the diffusion limit of conventional passive sensing method. OGMB-assisted active sensing method 
demonstrated in this work can improve the detection limit of analytes by an order of magnitude compared to that with passive sensing method.

Therefore, through this work, we have successfully met the three main objective of this research: (i) affordable and rapid fabrication of nanogap-rich structure to serve as a SERS substrate through an OGMB. (ii) concentrate chemicals or biological samples towards the nanogap-rich structure through an OGMB (iii) perform active sensing of analytes based on an OGMB-assisted SERS.

In the current experiment, fabrication of a nanogap-rich structure and the active analytes sensing are separated. For example, in this work nanoparticles are deposited at first on a substrate through the generation of OGMB to form nanogap-rich structure. Then the analyte solution is guided and concentrated on the nanogap-rich structure by generating another OGMB from analytes for active sensing. However, we can also combine these two processes to apply active sensing method. For example, nanoparticles solution can be premixed with analytes solution. In this process, surface of nanoparticles would be modified in order to bind analytes on the substrate. However, an OGMB can be used in order to deposit nanoparticles and simultaneously concentrate analytes on the surface. For example, this concept is demonstrated by Tantussi et. al. in their most recent article, where they premixed gold nanoparticles with a biological molecule known as extracellular membrane vehicles (EVs) in order to concentrate EVs through an OGMB. ${ }^{87}$

In this research, the laser used for nanogap-rich-structure fabrication (a continuous-wave laser with $1064 \mathrm{~nm}$ wavelength) and the laser used for Raman measurements in sensing application (diode laser of $785 \mathrm{~nm}$ ) are different. However, same laser can be used both for nanogap-rich-structure fabrication and Raman measurements in sensing application. For 
example, $785 \mathrm{~nm}$ laser which was used for the Raman measurements could be also used as a heat source for OGMB generation as well as nanogap-rich structure fabrication. There is no limit on the type of lasers for OGMB generation. In the literature, people have used different types of lasers for the generation of OGMB for different applications as reported in detailed in reference ${ }^{77}$ of this dissertation. The reason behind the using of different lasers in this experiment is, Raman system used in this work is located at another lab and we cannot build or shift our heating system incorporate with the Raman system used in this research. However, it is worth noting that, heating laser and exciting laser for Raman measurements in this work could be the same in order to simplify the experimental setup of this work. In order to prove this concept, we have used same laser (continuous wave laser with $1064 \mathrm{~nm}$ wavelength) to fabricate nanogap-rich structures and SERS measurement of analytes and performed real time active chemical sensing through OGMBassisted SERS on an optical fiber as reported in chaper 7.

It should be noted that, the analytes are immobilized on the nanogap-rich structures during active sensing because the analytes are printed on the nanogap-rich structure through OGMB. In our current experiment, we did not perform re-usable test of the SERS substrate, instead we used it as a one-time disposal SERS substrate due to the cost effective OGMBbased fabrication method. However, it would be possible to clean the analytes from the nanogap-rich structure by carefully choosing the right chemical treatment of the surface, which is beyond the scope of this work.

In conclusion, OGMB can be generated on any other type of sensors in order to apply active sensing method. Therefore, this OGMB-assisted active sensing method could be easily implemented to other type of sensing systems to improve the detection limit of a sensor. 
Through this method we can overcome the diffusion limit of conventional passive sensing method and thus can pave the way for advanced chemical and biosensing application.

\subsection{Future work}

As discussed in chapter 7, OGMB-based real time active sensing method can be applied for advanced chemical and biosensing application. We have already done some preliminary works on this concept which are reported in chapter 7. However, the detection limit of the fabricated OGMB-based nanogap-rich structures on optical fiber sensor could be further improved which is a future recommended work of this research.

Another working area could be the discrimination of chemical and biological sample by OGMB-assisted nanogap-rich structure. In this research, we have demonstrated the rapid detection of chemical and biological sample through our OGMB-based nanogap-rich structure. However, nanogap-rich structure can also be used for the discrimination of a combination of different chemicals and biological samples. Therefore, future recommended work in this area could be the rapid discrimination of different chemical and biological samples through an OGMB-based nanogap-rich structure. The detection of a chemical and biological sample can be performed by Raman spectroscopy. But, the discrimination of exact chemical or biological sample from the combination of several samples would not be performed by only observing the Raman peaks of analytes. Due to a significant number of overlapping peaks in the Raman spectra, it is difficult to detect exact bacteria, pathogens or any other chemical or bio-sample by only observing the Raman peak $^{122}$ as shown in Figure 8.1 (b). Therefore, in order to discriminate the exact chemical, pathogen or biological sample from the overlapping SERS spectra of analystes we need to 
perform an additional analysis known as principal components analysis (PCA) ${ }^{21,122}$ as shown in Figure 8.1 (a-c).
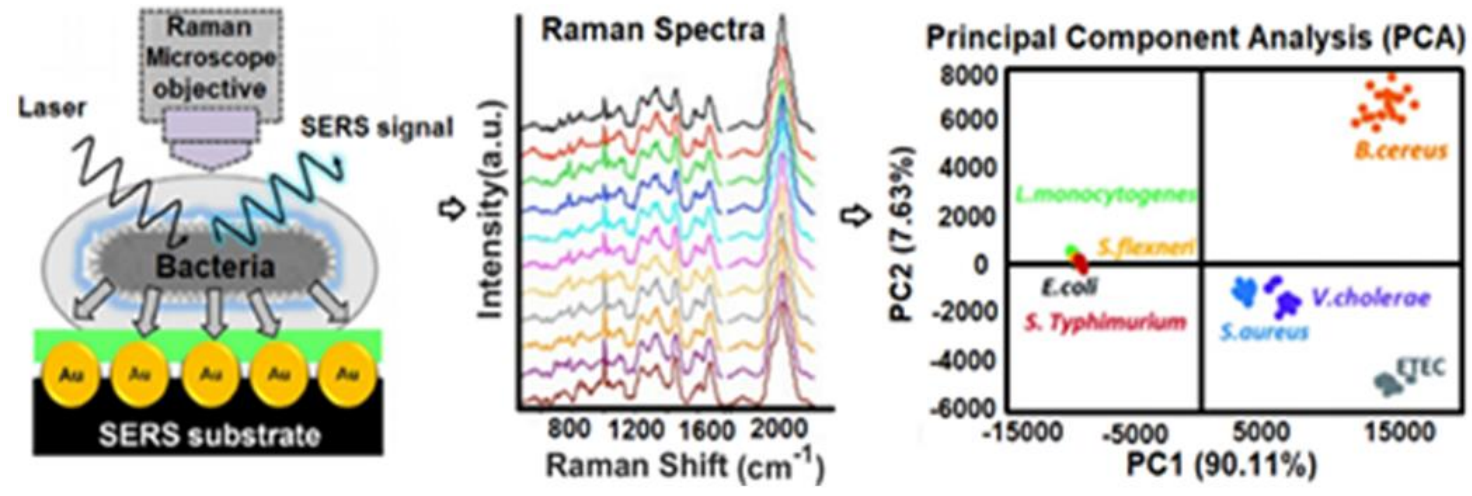

Figure 8.1 Detection and discrimination of pathogen (bacteria) by SERS measurement and principal components analysis (PCA). ${ }^{21}$

PCA is a powerful tool that will transform a number of correlated variables into several number of uncorrelated variables known as principal components, PC ${ }^{21,122}$ which are responsible for the corresponding chemical or biological samples. Through the combination of SERS measurements and PCA, we can successfully detect and discriminate exact bacteria, virus, toxins, parasites, toxic chemicals or any other pathogens as depicted in Figure $8.1(\mathrm{c})$. 


\section{BIBLOGRAPHY}

1. Mungkarndee, R., Tumcharern, G., Thiramanus, R., Techakriengkrai, I. \& Sukwattanasinitt, M. Fluorescence sensor arrays for identification of foodborne pathogens. Anal. Methods 7, 7431-7435 (2015).

2. Food Safety News | Breaking News about Food Safety. https://www.foodsafetynews.com/.

3. Cook, P. W. \& Nightingale, K. K. Use of omics methods for the advancement of food quality and food safety. - PubMed - NCBI. Anim. Front. 8, 33-41 (2018).

4. Water Contaminants: Health Effects of Air/Water Pollution - Water Fixers Plumbing \& Filtration. https://www.waterfixers.com/chemical-contaminants.html.

5. Center for Health Statistics, N. Health, United States, 2011. (2011).

6. Monat, C., Domachuk, P. \& Eggleton, B. J. Integrated optofluidics: A new river of light. Nature Photonics 1, 106-114 (2007).

7. Jia, Y., Jin, T. \& Liu, J. The influence of thermal exposure on the microstructure evolution of a nickel-base single crystal superalloy. Advanced Materials Research 418-420, 265-269 (2012).

8. Grieshaber, D., MacKenzie, R., Vörös, J. \& Reimhult, E. Electrochemical Biosensors - Sensor Principles and Architectures. Sensors 8, 1400-1458 (2008).

9. Lindholm-Sethson, B. et al. Electrochemical impedance spectroscopy in label-free biosensor applications: Multivariate data analysis for an objective interpretation. Analytical and Bioanalytical Chemistry 398, 2341-2349 (2010).

10. Alvarez, M. \& Lechuga, L. M. Microcantilever-based platforms as biosensing tools. Analyst 135, 827-836 (2010).

11. Gruber, K. et al. Cantilever array sensors detect specific carbohydrate-protein interactions with picomolar sensitivity. ACS Nano 5, 3670-3678 (2011).

12. Arlett, J. L., Myers, E. B. \& Roukes, M. L. Comparative advantages of mechanical biosensors. Nat. Nanotechnol. 6, 203-215 (2011).

13. Nie, S. \& Emory, S. R. Probing single molecules and single nanoparticles by surface-enhanced Raman scattering. Science 275, 1102-1106 (1997).

14. Grasselli, J. G., Walder, F., Petty, C. \& Kemeny, G. Industrial applications of Raman spectroscopy. J. Mol. Struct. 294, 207-210 (1993).

15. Tripp, R. A., Dluhy, R. A. \& Zhao, Y. Novel nanostructures for SERS biosensing. 
Nano Today 3, 31-37 (2008).

16. Cao, Y. C., Jin, R. \& Mirkin, C. A. Nanoparticles with Raman Spectroscopic Fingerprints for DNA and RNA Detection. Science 297, 1536-1540 (2002).

17. Vahimaa, P. Surface-Enhanced Raman Spectroscopy (SERS). (2000) doi:10.1002/MAME.201400391/ABSTRACT.

18. Li, D. W., Zhai, W. L., Li, Y. T. \& Long, Y. T. Recent progress in surface enhanced Raman spectroscopy for the detection of environmental pollutants. Microchimica Acta. 181, 23-43 (2014).

19. Chen, J. et al. Flexible and Adhesive Surface Enhance Raman Scattering Active Tape for Rapid Detection of Pesticide Residues in Fruits and Vegetables. Anal. Chem. 88, 2149-2155 (2016).

20. Mosier-Boss, P. Review of SERS Substrates for Chemical Sensing. Nanomaterials 7, 142 (2017).

21. Mosier-Boss, P. A. Review on SERS of bacteria. Biosensors 7, 51 (2017).

22. Karim, F., Vasquez, E. S. \& Zhao, C. Fabricated nanogap-rich plasmonic nanostructures through an optothermal surface bubble in a droplet. Opt. Lett. 43, 334-336 (2018).

23. Karim, F., Vasquez, E. S., Sun, Y. \& Zhao, C. Optothermal microbubble assisted manufacturing of nanogap-rich structures for active chemical sensing. Nanoscale 11, 20589-20597 (2019).

24. Maier, S. A. Plasmonics: Fundamentals and applications. (Springer US, 2007). doi:10.1007/0-387-37825-1.

25. Atwater, H. A. The Promise of Plasmonics. Sci. Am. 296, 56-62 (2007).

26. Polman, A. Applied physics: Plasmonics applied. Science 322, 868-869 (2008).

27. Sheehan, P. E. \& Whitman, L. J. Detection limits for nanoscale biosensors. Nano Lett. 5, 803-807 (2005).

28. Nair, P. R. \& Alam, M. A. Performance limits of nanobiosensors. Appl. Phys. Lett. 88, 233120 (2006).

29. Karim, F., Smith, T. B. \& Zhao, C. Review of optical detection of single molecules beyond the diffraction and diffusion limit using plasmonic nanostructures. $J$. Nanophotonics 12, 012504 (2017).

30. Squires, T. M., Messinger, R. J. \& Manalis, S. R. Making it stick: convection, reaction and diffusion in surface-based biosensors. Nat. Biotechnol. 26, 417-426 
(2008).

31. De Angelis, F. et al. Breaking the diffusion limit with super-hydrophobic delivery of molecules to plasmonic nanofocusing SERS structures. Nat. Photonics 5, 682687 (2011).

32. Nair, P. R. \& Alam, M. A. Screening-limited response of NanoBiosensors. Nano Lett. 8, 1281-1285 (2008).

33. McHale, G., Shirtcliffe, N. J. \& Newton, M. I. Super-hydrophobic and super-wetting surfaces: Analytical potential? Analyst 129, 284-287 (2004).

34. Mahadevan, L. \& Pomeau, Y. Rolling droplets. Phys. Fluids 11, 2449-2453 (1999).

35. Aussillous, P. \& Quéré, D. Liquid marbles. Nature 411, 924-927 (2001).

36. McHale, G., Aqil, S., Shirtcliffe, N. J., Newton, M. I. \& Erbil, H. Y. Analysis of droplet evaporation on a superhydrophobic surface. Langmuir 21, 11053-11060 (2005).

37. Yang, S., Dai, X., Stogin, B. B. \& Wong, T.-S. Ultrasensitive surface-enhanced Raman scattering detection in common fluids. Proc. Natl. Acad. Sci. U. S. A. 113, 268-273 (2016).

38. Escobedo, C., Brolo, A. G., Gordon, R. \& Sinton, D. Optofluidic concentration: Plasmonic nanostructure as concentrator and sensor. Nano Lett. 12, 1592-1596 (2012).

39. Eftekhari, F. et al. Nanoholes as nanochannels: flow-through plasmonic sensing. Anal. Chem. 81, 4308-4311 (2009).

40. Grubisha, D. S., Lipert, R. J., Park, H. Y., Driskell, J. \& Porter, M. D. Femtomolar Detection of Prostate-Specific Antigen: An Immunoassay Based on SurfaceEnhanced Raman Scattering and Immunogold Labels. Anal. Chem. 75, 5936-5943 (2003).

41. Lesniewski, A. et al. Antibody modified gold nanoparticles for fast and selective, colorimetric T7 bacteriophage detection. Bioconjug. Chem. 25, 644-648 (2014).

42. Wang, Y. \& Alocilja, E. C. Gold nanoparticle-labeled biosensor for rapid and sensitive detection of bacterial pathogens. J. Biol. Eng. 9, 16 (2015).

43. Anker, J. N. et al. Biosensing with plasmonic nanosensors. Nanoscience and Technology 7, 308-319 (2009)

44. Unser, S., Bruzas, I., He, J. \& Sagle, L. Localized surface plasmon resonance biosensing: Current challenges and approaches. Sensors 15, 15684-15716 (2015). 
45. Garcia-Guirado, J. et al. Overcoming Diffusion-Limited Biosensing by Electrothermoplasmonics. ACS Photonics 5, 3673-3679 (2018).

46. Gardiner, J. D. Practical Raman Spectroscopy Springer-Verlag. (1989).

47. Xu, M. et al. Heteroepitaxial Writing of Silicon-on-Sapphire Nanowires. Nano Lett. 16, 7317-7324 (2016).

48. Settle, F. A. Handbook of Instrumental Techniques for Analytical Chemistry. J. Liq. Chromatogr. Relat. Technol. 21, 3072-3076 (1998).

49. Chalmers, J. M., Edwards, H. G. M. \& Hargreaves, M. D. Infrared and Raman spectroscopy in forensic science. (Wiley, 2012).

50. Edwards, H. G. M. Modern Raman spectroscopy—a practical approach.Ewen Smith and Geoffrey Dent. John Wiley and Sons Ltd, Chichester, 2005. Pp. 210. ISBN 0 471496685 (cloth, hb); 0471497940 (pbk). J. Raman Spectrosc. 36, 835-835 (2005).

51. Bergholt, M. S., Serio, A. \& Albro, M. B. Raman Spectroscopy: Guiding Light for the Extracellular Matrix. Frontiers in Bioengineering and Biotechnology 7, 303 (2019).

52. Skoog, D. A, Holler, F. J. \& Nieman, T. A. Principles of Instrumental Analysis, Brooks/Cole. (1998).

53. Olander, D. P. Instrumental Methods of Analysis, Sixth Edition (Willard, Hobart H.; Merritt, Lynne L.; Dean, John A.; Settle, Frank A., Jr.). J. Chem. Educ. 61, A222 (1984).

54. Ember, K. J. I. et al. Raman spectroscopy and regenerative medicine: a review. npj Regen. Med. 2, (2017).

55. Kudelski, A. Analytical applications of Raman spectroscopy. Talanta 76, 1-8 (2008).

56. Sharma, B., Frontiera, R. R., Henry, A. I., Ringe, E. \& Van Duyne, R. P. SERS: Materials, applications, and the future. Materials Today 15, 16-25 (2012).

57. Jeanmaire, D. L. \& Van Duyne, R. P. Surface raman spectroelectrochemistry. Part I. Heterocyclic, aromatic, and aliphatic amines adsorbed on the anodized silver electrode. J. Electroanal. Chem. 84, 1-20 (1977).

58. Haynes, C. L., Farland, A. D. M. \& Duyne, R. P. Van. Surface-Enhanced Raman Spectroscopy New substrates and single- detection that are fueling interest in SERS Anal. Chem. 77, 338-346 (2005).

59. Kneipp, K. et al. Near-infrared surface-enhanced Raman scattering of trinitrotoluene 
on colloidal gold and silver. Spectrochim. Acta Part A Mol. Spectrosc. 51, 21712175 (1995).

60. Xu, H., Aizpurua, J., Käll, M. \& Apell, P. Electromagnetic contributions to singlemolecule sensitivity in surface-enhanced Raman scattering. Phys. Rev. E - Stat. Physics, Plasmas, Fluids, Relat. Interdiscip. Top. 62, 4318-4324 (2000).

61. Park, W. H. \& Kim, Z. H. Charge transfer enhancement in the SERS of a single molecule. Nano Lett. 10, 4040-4048 (2010).

62. Hu, M. et al. Gold nanofingers for molecule trapping and detection. J. Am. Chem. Soc. 132, 12820-12822 (2010).

63. Petti, L. et al. A plasmonic nanostructure fabricated by electron beam lithography as a sensitive and highly homogeneous SERS substrate for bio-sensing applications. Vib. Spectrosc. 82, 22-30 (2016).

64. Osinkina, L., Lohmüller, T., Jäckel, F. \& Feldmann, J. Synthesis of gold nanostar arrays as reliable, large-scale, homogeneous substrates for surface-enhanced Raman scattering imaging and spectroscopy. J. Phys. Chem. C 117, 22198-22202 (2013).

65. Liu, D. et al. Rapid Synthesis of Monodisperse Au Nanospheres through a Laser Irradiation-Induced Shape Conversion, Self-Assembly and Their Electromagnetic Coupling SERS Enhancement. Sci. Rep. 5, 7686 (2015).

66. Tian, S. et al. Aluminum nanocrystals: A sustainable substrate for quantitative SERS-Based DNA Detection. Nano Lett. 17, 5071-5077 (2017).

67. Polavarapu, L., Porta, A. La, Novikov, S. M., Coronado-Puchau, M. \& Liz-Marzán, L. M. Pen-on-paper approach toward the design of universal surface enhanced Raman scattering substrates. Small 10, 3065-3071 (2014).

68. Liu, D. et al. Black Gold: Plasmonic Colloidosomes with Broadband Absorption Self-Assembled from Monodispersed Gold Nanospheres by Using a Reverse Emulsion System. Angew. Chemie - Int. Ed. 54, 9596-9600 (2015).

69. Liu, D. et al. Capillary Gradient-Induced Self-Assembly of Periodic Au Spherical Nanoparticle Arrays on an Ultralarge Scale via a Bisolvent System at Air/Water Interface. Adv. Mater. Interfaces 4, 1600976 (2017).

70. Rajeeva, B. B. et al. "Point-and-Shoot" Synthesis of Metallic Ring Arrays and Surface-Enhanced Optical Spectroscopy. Adv. Opt. Mater. 6, 1701213 (2018).

71. Xie, Y. et al. In Situ Fabrication of 3D Ag@ZnO Nanostructures for Microfluidic Surface-Enhanced Raman Scattering Systems. ACS Nano 8, 12175-12184 (2014).

72. Chen, X. et al. Split-Wedge Antennas with Sub-5 nm Gaps for Plasmonic Nanofocusing. Nano Lett. 16, 7849-7856 (2016). 
73. Xie, Y. et al. Optoacoustic tweezers: A programmable, localized cell concentrator based on opto-thermally generated, acoustically activated, surface bubbles. Lab Chip 13, 1772-1779 (2013).

74. Zhao, C. et al. Theory and experiment on particle trapping and manipulation via optothermally generated bubbles. Lab Chip 14, 384-391 (2014).

75. Fan, Q., Hu, W. \& Ohta, A. T. Laser-induced microbubble poration of localized single cells. Lab Chip 14, 1572-1578 (2014).

76. Fan, Q., Hu, W. \& Ohta, A. T. Efficient single-cell poration by microsecond laser pulses. Lab Chip 15, 581-588 (2015).

77. Xie, Y. \& Zhao, C. An optothermally generated surface bubble and its applications. Nanoscale 9, 6622-6631 (2017).

78. Setoura, K., Ito, S. \& Miyasaka, H. Stationary bubble formation and Marangoni convection induced by $\mathrm{CW}$ laser heating of a single gold nanoparticle. Nanoscale 9, 719-730 (2017).

79. Greenberg, E., Armon, N., Kapon, O., Ben-Ishai, M. \& Shpaisman, H. Nanostructure and Mechanism of Metal Deposition by a Laser-Induced Photothermal Reaction. Adv. Mater. Interfaces 6, 1900541 (2019).

80. Bangalore Rajeeva, B. et al. High-Resolution Bubble Printing of Quantum Dots. ACS Appl. Mater. Interfaces 9, 16725-16733 (2017).

81. Donner, J. S., Baffou, G., McCloskey, D. \& Quidant, R. Plasmon-assisted optofluidics. ACS Nano. 5, 5457-5462 (2011).

82. Roxworthy, B. J., Bhuiya, A. M., Vanka, S. P. \& Toussaint, K. C. Understanding and controlling plasmon-induced convection. Nat. Commun. 5, 3173 (2014).

83. Zheng, Y. et al. Accumulating microparticles and direct-writing micropatterns using a continuous-wave laser-induced vapor bubble. Lab Chip 11, 3816-3820 (2011).

84. Lin, L. et al. Bubble-Pen Lithography. Nano Lett. 16, 701-708 (2016).

85. Bangalore Rajeeva, B. et al. High-Resolution Bubble Printing of Quantum Dots. ACS Appl. Mater. Interfaces 9, 16725-16733 (2017).

86. Fujii, S. et al. Fabrication and Placement of a Ring Structure of Nanoparticles by a Laser-Induced Micronanobubble on a Gold Surface. Langmuir 27, 8605-8610 (2011).

87. Tantussi, F. et al. Long-Range Capture and Delivery of Water-Dispersed Nanoobjects by Microbubbles Generated on 3D Plasmonic Surfaces. ACS Nano 12, 4116-4122 (2018). 
88. Zhao, C., Liu, Y., Zhao, Y., Fang, N. \& Huang, T. J. A reconfigurable plasmofluidic lens. Nat. Commun. 4, 2305 (2013).

89. Meng, F. et al. Vapor-Enabled Propulsion for Plasmonic Photothermal Motor at the Liquid/Air Interface. J. Am. Chem. Soc. 139, 12362-12365 (2017).

90. Zhang, K. et al. Laser-induced thermal bubbles for microfluidic applications. Lab Chip 11, 1389-1395 (2011).

91. Fischer, B. J. Particle convection in an evaporating colloidal droplet. Langmuir 18, 60-67 (2002).

92. Kong, X. et al. Optofluidic sensing from inkjet-printed droplets: The enormous enhancement by evaporation-induced spontaneous flow on photonic crystal biosilica. Nanoscale 8, 17285-17294 (2016).

93. Cheung, M. et al. Raman Analysis of Dilute Aqueous Samples by Localized Evaporation of Submicroliter Droplets on the Tips of Superhydrophobic Copper Wires. Anal. Chem. 88, 4541-4547 (2016).

94. Trantum, J. R., Baglia, M. L., Eagleton, Z. E., Mernaugh, R. L. \& Haselton, F. R. Biosensor design based on Marangoni flow in an evaporating drop. Lab Chip 14, 315-324 (2014).

95. Ristenpart, W. D., Kim, P. G., Domingues, C., Wan, J. \& Stone, H. A. Influence of substrate conductivity on circulation reversal in evaporating drops. Phys. Rev. Lett. 99, (2007).

96. Hu, H. \& Larson, R. G. Analysis of the microfluid flow in an evaporating sessile droplet. Langmuir 21, 3963-3971 (2005).

97. Deegan, R. D. et al. Capillary flow as the cause of ring stains from dried liquid drops. Nature 389, 827-829 (1997).

98. Wu, C., Chen, E. \& Wei, J. Surface enhanced Raman spectroscopy of Rhodamine $6 \mathrm{G}$ on agglomerates of different-sized silver truncated nanotriangles. Colloids Surfaces A Physicochem. Eng. Asp. 506, 450-456 (2016).

99. Tian, F., Bonnier, F., Casey, A., Shanahan, A. E. \& Byrne, H. J. Surface enhanced Raman scattering with gold nanoparticles: effect of particle shape. Anal. Methods 6 , 9116-9123 (2014).

100. Zong, X., Zhu, R. \& Guo, X. Nanostructured gold microelectrodes for SERS and EIS measurements by incorporating $\mathrm{ZnO}$ nanorod growth with electroplating. Sci. Rep. 5, 16454 (2015).

101. Le Ru, E. C., Blackie, E., Meyer, M. \& Etchegoin, P. G. Surface Enhanced Raman Scattering Enhancement Factors: A Comprehensive Study. J. Phys. Chem. C 111, 
13794-13803 (2007).

102. Kahraman, M., Daggumati, P., Kurtulus, O., Seker, E. \& Wachsmann-Hogiu, S. Fabrication and Characterization of Flexible and Tunable Plasmonic Nanostructures. Sci. Rep. 3, 3396 (2013).

103. Ding, S.-Y., You, E.-M., Tian, Z.-Q. \& Moskovits, M. Electromagnetic theories of surface-enhanced Raman spectroscopy. Chem. Soc. Rev. 46, 4042-4076 (2017).

104. Merlen, A., Lagugné-Labarthet, F. \& Harté, E. Surface-Enhanced Raman and Fluorescence Spectroscopy of Dye Molecules Deposited on Nanostructured Gold Surfaces. J. Phys. Chem. C 114, 12878-12884 (2010).

105. Mo, Y., Lei, J., Li, X. \& Wachter, P. Surface enhanced Raman scattering of rhodamine 6G and dye 1555 adsorbed on roughened copper surfaces. Solid State Commun. 66, 127-131 (1988).

106. Chi, T. T. K., Le, N. T., Hien, B. T. T., Trung, D. Q. \& Liem, N. Q. Preparation of SERS Substrates for the Detection of Organic Molecules at Low Concentration. Commun. Phys. 26, 261 (2017).

107. Baffou, G., Polleux, J., Rigneault, H. \& Monneret, S. Super-Heating and MicroBubble Generation around Plasmonic Nanoparticles under cw Illumination. J. Phys. Chem. C 118, 4890-4898 (2014).

108. Setoura, K., Ito, S. \& Miyasaka, H. Stationary bubble formation and Marangoni convection induced by $\mathrm{CW}$ laser heating of a single gold nanoparticle. Nanoscale $\mathbf{9}$, 719-730 (2017).

109. Chikazawa, J., Uwada, T., Furube, A. \& Hashimoto, S. Flow-Induced Transport via Optical Heating of a Single Gold Nanoparticle. J. Phys. Chem. C 123, 4512-4522 (2019).

110. Farber, J. M. \& Peterkin, P. I. Listeria monocytogenes, a food-borne pathogen. Microbiological Reviews. 55, 476-511 (1991).

111. F. Karim, E. Vasquez, Y. Sun, C. Z. Active and ultrasensitive chemical and biosensing through optothermally generated microbubble. Conference on Lasers and Electro-Optics (CLEO), 2020. San Jose, CA, USA.

112. Wang, J. et al. Rapid detection of Listeria monocytogenes in milk using confocal micro-Raman spectroscopy and chemometric analysis. Int. J. Food Microbiol. 204, 66-74 (2015).

113. Kashish, Soni, D. K., Mishra, S. K., Prakash, R. \& Dubey, S. K. Label-free impedimetric detection of Listeria monocytogenes based on poly-5-carboxy indole modified ssDNA probe. J. Biotechnol. 200, 70-76 (2015). 
114. Wang, C. et al. A rapid SERS method for label-free bacteria detection using polyethylenimine-modified Au-coated magnetic microspheres and $\mathrm{Au} @ \mathrm{Ag}$ nanoparticles. Analyst 141, 6226-6238 (2016).

115. Wu, Y. et al. Label-free Acoustic and Optical Biosensors Playing on Evanescent Waves. PIERS Proceedings. 1756-1771 (2013).

116. Sirbuly, D. J., Tao, A., Law, M., Fan, R. \& Yang, P. Multifunctional Nanowire Evanescent Wave Optical Sensors. Adv. Mater. 19, 61-66 (2007).

117. Liu, Y., Xu, S., Li, H., Jian, X. \& Xu, W. Localized and propagating surface plasmon co-enhanced Raman spectroscopy based on evanescent field excitation. Chem. Commun. 47, 3784-3786 (2011).

118. Gu, Y., Li, H., Xu, S., Liu, Y. \& Xu, W. Evanescent field excited plasmonic nanoantenna for improving SERS signal. Phys. Chem. Chem. Phys. 15, 15494-15498 (2013).

119. Chen, P. \& Shakhnovich, E. I. Thermal adaptation of viruses and bacteria. Biophys. J. 98, 1109-1118 (2010).

120. Sánchez-Solís, A. et al. Print metallic nanoparticles on a fiber probe for 1064-nm surface-enhanced Raman scattering. Opt. Lett. 44, 4997-5000 (2019).

121. McCreery, R. L. Raman spectroscopy for chemical analysis. (John Wiley \& Sons, 2000).

122. Kotanen, C. N., Martinez, L., Alvarez, R. \& Simecek, J. W. Surface enhanced Raman scattering spectroscopy for detection and identification of microbial pathogens isolated from human serum. Sens. Bio-Sensing Res. 8, 20-26 (2016). 